\title{
The Enantioselective Intermolecular Saegusa Allylation
}

Ji Liu, Edward M. Laguna, Arun Raj Kizhakkayil Mangadan, Kyoungmin Kang, Aaron Aponick*

Florida Center for Heterocyclic Compounds, Department of Chemistry, University of Florida, Gainesville, Florida 32611, United States

Email: aponick@ufl.edu

\section{Supporting Information}

\section{Table of Contents}

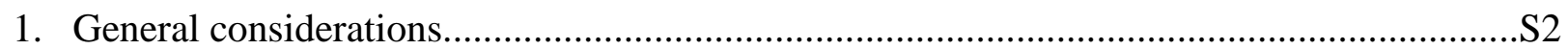

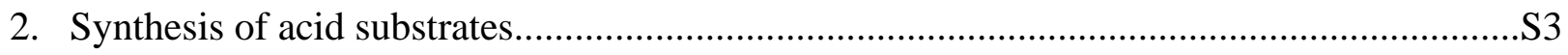

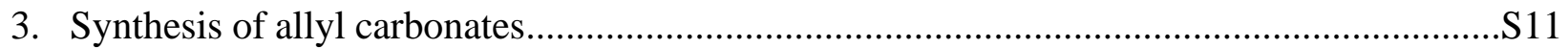

4. Scope studies of the enantioselective intermolecular allylation.......................................S14

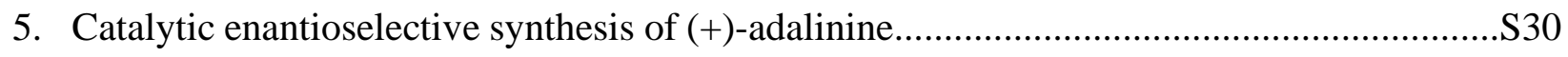

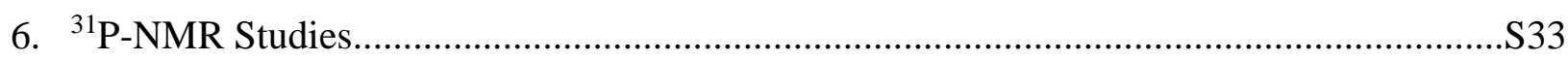

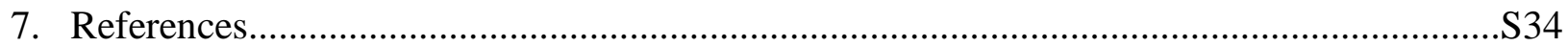

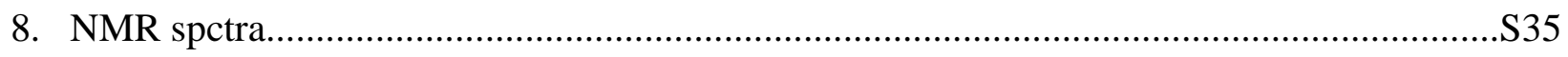




\section{General Considerations}

All reactions were carried out under an atmosphere of dry nitrogen unless otherwise specified. Anhydrous solvents were transferred via syringe to flame-dried glassware, which had been cooled under a stream of dry nitrogen. Anhydrous tetrahydrofuran (THF), dichloromethane $\left(\mathrm{CH}_{2} \mathrm{Cl}_{2}\right)$, acetonitrile $\left(\mathrm{CH}_{3} \mathrm{CN}\right)$ and toluene $(\mathrm{PhMe})$ were dried using a mBraun solvent purification system. All other reagents were ordered from Sigma-Aldrich and used without any further purification. Analytical thin layer chromatography (TLC) was performed using $250 \mu \mathrm{m}$ Silica Gel $60 \AA$ precoated plates. Flash column chromatography was performed using 230-400 Mesh $60 \AA$ Silica Gel. The eluents employed are reported as volume:volume percentages. Proton nuclear magnetic resonance ( ${ }^{1} \mathrm{H}$ NMR) spectra were recorded using Varian Unity Mercury 300 MHz, BrukerBioSpin GmbH $400 \mathrm{MHz}$, Varian Unity Mercury $500 \mathrm{MHz}$ and BrukerBioSpin $\mathrm{GmbH} 600 \mathrm{MHz}$. Chemical shifts $(\delta)$ are reported in parts per million (ppm) downfield relative to tetramethylsilane (TMS, $0.0 \mathrm{ppm}$ ) or $\mathrm{CDCl}_{3}(7.26 \mathrm{ppm}),\left(\mathrm{CD}_{3}\right)_{2} \mathrm{CO}(2.05 \mathrm{ppm}), \mathrm{CD}_{3} \mathrm{OD}$ (3.31 ppm). Coupling constants $(J)$ are reported in Hz. Multiplicities are reported using the following abbreviations: s, singlet; d, doublet; t, triplet; q, quartet; m, multiplet; br, broad; Carbon-13 nuclear magnetic resonance $\left({ }^{13} \mathrm{C} \mathrm{NMR}\right)$ spectra were recorded using Varian Unity Mercury $300 \mathrm{MHz}$, BrukerBioSpin GmbH 400 MHz, Varian Unity Mercury 500 MHz and BrukerBioSpin GmbH 600 $\mathrm{MHz}$ at $75 \mathrm{MHz}, 100 \mathrm{MHz}, 125 \mathrm{MHz} 150 \mathrm{MHz}$ respectively. Chemical shifts are reported in ppm relative to the carbon resonance of $\mathrm{CDCl}_{3}$ (77.16 ppm), $\left(\mathrm{CD}_{3}\right)_{2} \mathrm{CO}$ (206.7, $\left.29.9 \mathrm{ppm}\right), \mathrm{CD}_{3} \mathrm{OD}$ (49.0 ppm). Fluorine-19 ( ${ }^{19} \mathrm{~F}$ NMR) nuclear magnetic resonance spectra were recorded using Varian Unity Mercury 300 spectrometer at $300 \mathrm{MHz}$. The ${ }^{19} \mathrm{~F}$ NMR chemical shifts were calibrated using an external reference sample of Trifluorotoluene, $\mathrm{C}_{6} \mathrm{H}_{5} \mathrm{CF}_{3}$ in DMSO-d $\mathrm{d}_{6}(\delta-63.72 \mathrm{ppm}$ ). Specific Optical rotations were obtained on a JASCO P - 2000 Series Polarimeter (wavelength $=$ $589 \mathrm{~nm}$ ). High resolution mass spectra (HRMS) were obtained by Mass Spectrometry Core Laboratory at the University of Florida and are reported as $\mathrm{m} / \mathrm{e}$ (relative ratio). The Instrument Agilent 6200 was used for ESI-TOF analysis and an IonSense DART ET-100 ionization source was coupled to the instrument for DART-TOF analysis. Accurate $\mathrm{m} / \mathrm{z}$ are reported for the molecular ion $[\mathrm{M}+\mathrm{H}]^{+}$or a suitable fragment ion. All chemicals were purchased from suppliers and used as received unless noted otherwise. 


\section{Synthesis of Acid Substrates}

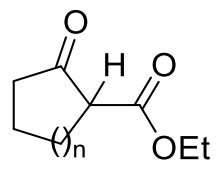

1) $\mathrm{Mel}, \mathrm{DMSO}$

$\mathrm{K}_{3} \mathrm{PO}_{4}, \mathrm{rt}, 12 \mathrm{~h}$

2) $\mathrm{NaOH}, \mathrm{MeOH} / \mathrm{H}_{2} \mathrm{O}$ $\mathrm{rt}, 12 \mathrm{~h}$

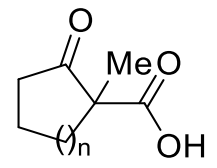<smiles>O=C(O)C1(C(=O)O)CCCCC1=O</smiles>

$1 \mathrm{a}$

1-methyl-2-oxocyclohexane-1-carboxylic acid (1a). Ethyl 2-oxocyclohexane-1carboxylate (1.70 g, $10 \mathrm{mmol}$, purchased from Sigma-Aldrich) was dissolved in DMSO (10 mL). $\mathrm{K}_{3} \mathrm{PO}_{4}(4.2 \mathrm{~g}, 20 \mathrm{mmol})$ and $\mathrm{MeI}(2.8 \mathrm{~g}, 20 \mathrm{mmol})$ were added subsequently. The resulting mixture was allowed to stir at room temperature for $12 \mathrm{~h}$ and then diluted with a mixture of ether and hexanes $(50 \mathrm{~mL} / 50 \mathrm{~mL})$. The organic mixture was washed with brine $(2 \times 50 \mathrm{~mL})$, dried over $\mathrm{Na}_{2} \mathrm{SO}_{4}$ and concentrated under reduced pressure to give the crude product which was directly submitted to the next step without further purification.

The crude product obtained from above was dissolved in $\mathrm{MeOH}(20 \mathrm{~mL})$. Aqueous $\mathrm{NaOH}$ (20 $\mathrm{mL}, 20 \%$ in wt) was added and the reaction mixture was allowed to stir at room temperature for 12 hour and then cooled to $0{ }^{\circ} \mathrm{C}$ in an ice bath. The reaction mixture was carefully acidified with $\mathrm{HCl}(6 \mathrm{M})$ to $\mathrm{pH}=3$ and then extracted with ether $(3 \times 50 \mathrm{~mL})$. The organic extracts were combined, washed with brine $(50 \mathrm{~mL})$, dried over $\mathrm{Na}_{2} \mathrm{SO}_{4}$ and concentrated under reduced pressure to give the crude product which was further purified by column chromatography (20\% to $50 \% \mathrm{Et}_{2} \mathrm{O} /$ hexanes gradient) to give title product in $65 \%$ yield $(1.01 \mathrm{~g})$ over two steps as a white solid. $\mathrm{R}_{\mathrm{f}}=0.10\left(50 \% \mathrm{EtOAc} /\right.$ hexanes, $\mathrm{KMnO}_{4}$ stain). ${ }^{1} \mathrm{H} \mathrm{NMR}\left(500 \mathrm{MHz}, \mathrm{CDCl}_{3}\right) \delta 2.58$ (dddd, $J=15.6,9.7,6.3,3.0 \mathrm{~Hz}, 1 \mathrm{H}), 2.52-2.44(\mathrm{~m}, 2 \mathrm{H}), 2.15-1.96(\mathrm{~m}, 1 \mathrm{H}), 1.81-1.49(\mathrm{~m}, 4 \mathrm{H}), 1.37$ 
(s, 3H). ${ }^{13} \mathrm{C}$ NMR $\left(125 \mathrm{MHz}, \mathrm{CDCl}_{3}\right) \delta 208.4,179.3,56.9,40.4,37.9,27.3,22.4,21.2$. HRMS

(ESI) $\mathrm{m} / \mathrm{z}:[\mathrm{M}+\mathrm{H}]+$ calcd for $\mathrm{C}_{8} \mathrm{H}_{12} \mathrm{O}_{3}$ 157.0859; Found 157.0855.

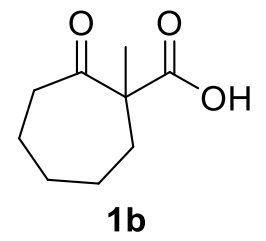

1-methyl-2-oxocycloheptane-1-carboxylic acid (1b). Ethyl 2-oxocycloheptane-1carboxylate (1.84 g, $10 \mathrm{mmol}$, purchased from Sigma-Aldrich) was dissolved in DMSO (10 mL). $\mathrm{K}_{3} \mathrm{PO}_{4}(4.2 \mathrm{~g}, 20 \mathrm{mmol})$ and $\mathrm{MeI}(2.8 \mathrm{~g}, 20 \mathrm{mmol})$ were added subsequently. The resulting mixture was allowed to stir at room temperature for $12 \mathrm{~h}$ and then diluted with a mixture of ether and hexanes $(50 \mathrm{~mL} / 50 \mathrm{~mL})$. The organic mixture was washed with brine $(2 \times 50 \mathrm{~mL})$, dried over $\mathrm{Na}_{2} \mathrm{SO}_{4}$ and concentrated under reduced pressure to give the crude product which was directly submitted to next step without further purification.

The crude product obtained from above was dissolved in $\mathrm{MeOH}(20 \mathrm{~mL})$. Aqueous $\mathrm{NaOH}$ (20 $\mathrm{mL}, 20 \%$ in wt) was added and the reaction mixture was allowed to stir at room temperature for 12 hour and then cooled to $0{ }^{\circ} \mathrm{C}$ in an ice bath. The reaction mixture was carefully acidified with $\mathrm{HCl}(6 \mathrm{M})$ to $\mathrm{pH}=3$ and then extracted with ether $(3 \times 50 \mathrm{~mL})$. The organic extracts were combined, washed with brine $(50 \mathrm{~mL})$, dried over $\mathrm{Na}_{2} \mathrm{SO}_{4}$ and concentrated under reduced pressure to give the crude product which was further purified by column chromatography (20\% to $50 \% \mathrm{Et}_{2} \mathrm{O} /$ hexanes gradient) to give title product in $61 \%$ yield $(1.04 \mathrm{~g})$ over two steps as a white solid. $\mathrm{R}_{\mathrm{f}}=0.10$ (50\% EtOAc/hexanes, $\mathrm{KMnO}_{4}$ stain). ${ }^{1} \mathrm{H} \mathrm{NMR}\left(500 \mathrm{MHz}, \mathrm{CDCl}_{3}\right) \delta 2.61$ (ddd, $J$ $=12.8,9.6,3.2 \mathrm{~Hz}, 1 \mathrm{H}), 2.41(\mathrm{ddd}, J=11.8,8.9,2.4 \mathrm{~Hz}, 1 \mathrm{H}), 2.19-1.90(\mathrm{~m}, 1 \mathrm{H}), 1.75-1.54$ (m, 3H), $1.46(\mathrm{~m}, 4 \mathrm{H}), 1.21(\mathrm{~s}, 3 \mathrm{H}) .{ }^{13} \mathrm{C} \mathrm{NMR}\left(125 \mathrm{MHz}, \mathrm{CDCl}_{3}\right) \delta$ 210.8, 178.77, 58.5, 41.7, 
35.1, 30.0, 25.7, 24.7, 21.3. HRMS (ESI) m/z: [M+H]+ calcd for $\mathrm{C}_{9} \mathrm{H}_{14} \mathrm{O}_{3}$ 171.1016; Found 171.1020 .
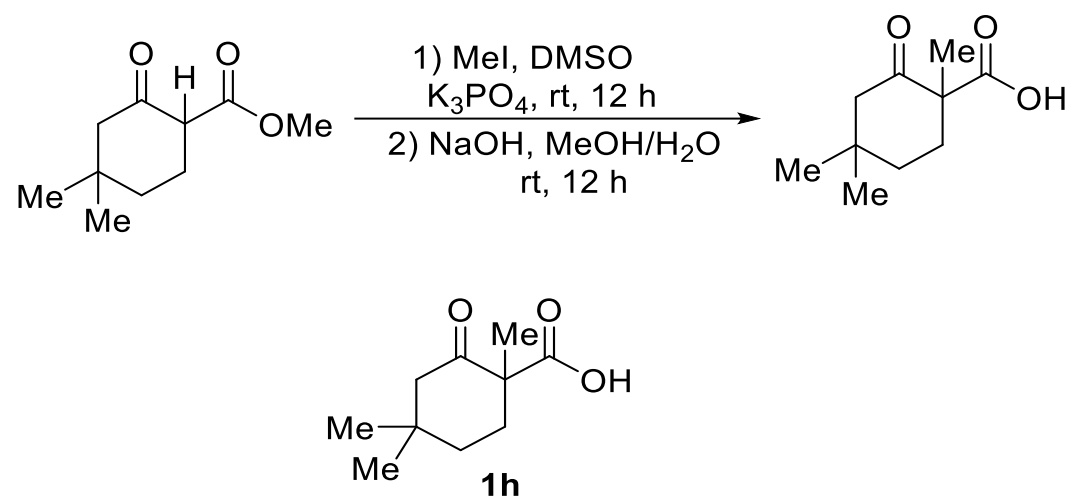

1,4,4-trimethyl-2-oxocyclohexane-1-carboxylic acid (1h). Ethyl 4,4-dimethyl-2oxocyclohexane-1-carboxylate ${ }^{1}(1.98 \mathrm{~g}, 10 \mathrm{mmol})$ was dissolved in DMSO (10 mL). $\mathrm{K}_{3} \mathrm{PO}_{4}(4.2$ g, $20 \mathrm{mmol})$ and MeI (2.8 g, $20 \mathrm{mmol})$ were added subsequently. The resulting mixture was allowed to stir at room temperature for $12 \mathrm{~h}$ and then diluted with a mixture of ether and hexanes $(50 \mathrm{~mL} / 50 \mathrm{~mL})$. The organic mixture was washed with brine $(2 \times 50 \mathrm{~mL})$, dried over $\mathrm{Na}_{2} \mathrm{SO}_{4}$ and concentrated under reduced pressure to give the crude product which was directly submitted to next step without further purification.

The crude product obtained from above was dissolved in $\mathrm{MeOH}(20 \mathrm{~mL})$. Aqueous $\mathrm{NaOH}$ (20 mL, 20\% in wt) was added and the reaction mixture was allowed to stir at room temperature for 12 hour and then cooled to $0{ }^{\circ} \mathrm{C}$ in an ice bath. The reaction mixture was carefully acidified with $\mathrm{HCl}(6 \mathrm{M})$ to $\mathrm{pH}=3$ and then extracted with ether $(3 \times 50 \mathrm{~mL})$. The organic extracts were combined, washed with brine $(50 \mathrm{~mL})$, dried over $\mathrm{Na}_{2} \mathrm{SO}_{4}$ and concentrated under reduced pressure to give the crude product which was further purified by column chromatography (20\% to $50 \% \mathrm{Et}_{2} \mathrm{O} /$ hexanes gradient) to give title product in $67 \%$ yield $(1.23 \mathrm{~g})$ over two steps as a white solid. $\mathrm{R}_{\mathrm{f}}=0.10\left(50 \%\right.$ EtOAc/hexanes, $\mathrm{KMnO}_{4}$ stain $) .{ }^{1} \mathrm{H} \mathrm{NMR}\left(500 \mathrm{MHz}, \mathrm{CDCl}_{3}\right) \delta 2.53-2.37$ (m, 2H), $2.26(\mathrm{dd}, J=13.5,2.0 \mathrm{~Hz}, 1 \mathrm{H}), 1.79-1.64(\mathrm{~m}, 2 \mathrm{H}), 1.61-1.48(\mathrm{~m}, 1 \mathrm{H}), 1.38(\mathrm{~s}, 3 \mathrm{H})$, 
$1.06(\mathrm{~s}, 3 \mathrm{H}), 0.93(\mathrm{~s}, 3 \mathrm{H}) .{ }^{13} \mathrm{C} \mathrm{NMR}\left(125 \mathrm{MHz}, \mathrm{CDCl}_{3}\right) \delta 208.2,178.8,55.7,53.2,36.6,35.5,33.4$, 30.5, 26.3, 21.1. HRMS (ESI) m/z: [M+H]+ calcd for $\mathrm{C}_{10} \mathrm{H}_{16} \mathrm{O}_{3}$ 185.1172; Found 185.1179.<smiles>O=C(O)C1CCc2ccccc2C1=O</smiles>

$1 p$

2-methyl-1-oxo-1,2,3,4-tetrahydronaphthalene-2-carboxylic acid (1p). Synthesized using a reported procedure in $45 \%$ yield as a light brown solid. The ${ }^{1} \mathrm{H}$ NMR and ${ }^{13} \mathrm{C}$ NMR spectra matched with reported data. ${ }^{2}$

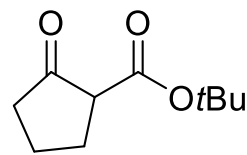

S1
1) $\mathrm{RX}, \mathrm{DMSO}$

$\frac{\mathrm{K}_{3} \mathrm{PO}_{4}, \mathrm{rt}, 12 \mathrm{~h}}{\text { 2) } \mathrm{TFA}, \mathrm{DCM}}$ $\mathrm{rt}, 3 \mathrm{~h}$

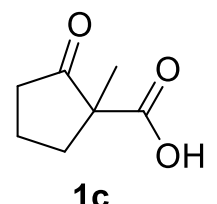

1-methyl-2-oxocyclopentane-1-carboxylic acid (1c). Tert-butyl 2-oxocyclopetane-1carboxylate ${ }^{3,4}(1.84 \mathrm{~g}, 10 \mathrm{mmol})$ was dissolved in DMSO $(10 \mathrm{~mL}) . \mathrm{K}_{3} \mathrm{PO}_{4}(4.2 \mathrm{~g}, 20 \mathrm{mmol})$ and MeI ( $2.8 \mathrm{~g}, 20 \mathrm{mmol})$ were added subsequently. The resulting mixture was allowed to stir at room temperature for $12 \mathrm{~h}$ and then diluted with a mixture of ether and hexanes $(50 \mathrm{~mL} / 50 \mathrm{~mL})$. The organic mixture was washed with brine $(2 \times 50 \mathrm{~mL})$, dried over $\mathrm{Na}_{2} \mathrm{SO}_{4}$ and concentrated under reduced pressure to give the crude product which was directly submitted to next step without further purification.

The crude product obtained from above was dissolved in DCM $(10 \mathrm{~mL})$ and cooled to 0 ${ }^{\circ} \mathrm{C}$ in an ice bath. TFA $(5 \mathrm{~mL})$ was added and the reaction mixture was allowed to stir at room temperature for 3 hours and then concentrated under reduced pressure to give the crude product 
which was further purified by column chromatography ( $20 \%$ to $50 \% \mathrm{Et}_{2} \mathrm{O} /$ hexanes gradient) to give title product in $74 \%$ yield $(1.10 \mathrm{~g})$ over two steps as a colorless oil. $\mathrm{R}_{\mathrm{f}}=0.10(50 \%$ EtOAc/hexanes, $\mathrm{KMnO}_{4}$ stain). ${ }^{1} \mathrm{H}$ NMR $\left(500 \mathrm{MHz}, \mathrm{CDCl}_{3}\right) \delta 2.63-2.51(\mathrm{~m}, 1 \mathrm{H}), 2.49-2.40$ (m, 1H), $2.41-2.29(\mathrm{~m}, 1 \mathrm{H}), 2.16-2.02(\mathrm{~m}, 1 \mathrm{H}), 1.99-1.86(\mathrm{~m}, 2 \mathrm{H}), 1.32(\mathrm{~s}, 3 \mathrm{H}) .{ }^{13} \mathrm{C}$ NMR $\left(125 \mathrm{MHz}, \mathrm{CDCl}_{3}\right) \delta 215.7,178.2,55.8,37.6,35.9,19.5,19.3 . \mathrm{HRMS}(\mathrm{ESI}) \mathrm{m} / \mathrm{z}:[\mathrm{M}+\mathrm{H}]+$ calcd for $\mathrm{C}_{7} \mathrm{H}_{10} \mathrm{O}_{3}$ 143.0703; Found 143.0710.

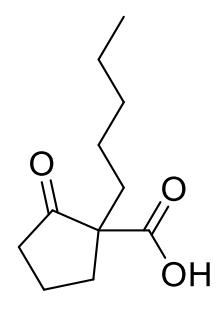

$1 q$

2-oxo-1-pentylcyclopentane-1-carboxylic acid (1q). Tert-butyl 2-oxocyclopetane-1carboxylate ${ }^{3,4}(1.84 \mathrm{~g}, 10 \mathrm{mmol})$ was dissolved in DMSO $(10 \mathrm{~mL}) . \mathrm{K}_{3} \mathrm{PO}_{4}(4.2 \mathrm{~g}, 20 \mathrm{mmol})$ and $\mathrm{n}-$ pentyl iodide $(4.0 \mathrm{~g}, 20 \mathrm{mmol})$ were added subsequently. The resulting mixture was allowed to stir at room temperature for $12 \mathrm{~h}$ and then diluted with a mixture of ether and hexanes $(50 \mathrm{~mL} / 50 \mathrm{~mL})$. The organic mixture was washed with brine $(2 \times 50 \mathrm{~mL})$, dried over $\mathrm{Na}_{2} \mathrm{SO}_{4}$ and concentrated under reduced pressure to give the crude product which was directly submitted to next step without further purification.

The crude product obtained from above was dissolved in DCM $(10 \mathrm{~mL})$ and cooled to 0 ${ }^{\circ} \mathrm{C}$ in an ice bath. TFA $(5 \mathrm{~mL})$ was added and the reaction mixture was allowed to stir at room temperature for 3 hours and then concentrated under reduced pressure to give the crude product which was further purified by column chromatography ( $20 \%$ to $50 \% \mathrm{Et}_{2} \mathrm{O} /$ hexanes gradient) to give title product in $66 \%$ yield $(1.30 \mathrm{~g})$ over two steps as a colorless oil. $\mathrm{R}_{\mathrm{f}}=0.50(50 \%$ 
EtOAc/hexanes, $\mathrm{KMnO}_{4}$ stain). ${ }^{1} \mathrm{H}$ NMR $\left(300 \mathrm{MHz}, \mathrm{CDCl}_{3}\right) \delta 2.56-2.43(\mathrm{~m}, 1 \mathrm{H}), 2.42-2.22$ (m, 1H), $2.08-1.79(\mathrm{~m}, 4 \mathrm{H}), 1.59-1.45(\mathrm{~m}, 1 \mathrm{H}), 1.38-1.13(\mathrm{~m}, 6 \mathrm{H}), 0.84(\mathrm{t}, J=6.6 \mathrm{~Hz}, 3 \mathrm{H})$. ${ }^{13} \mathrm{C}$ NMR $\left(125 \mathrm{MHz}, \mathrm{CDCl}_{3}\right) \delta 215.1,176.6,60.4,37.8,33.9,32.2,31.9,24.3,22.3,19.4,13.9$. HRMS (ESI) m/z: [M+H]+ calcd for $\mathrm{C}_{11} \mathrm{H}_{18} \mathrm{O}_{3}$ 199.1329; Found 199.1338.<smiles>CCCCOC(=O)C1CCCCC1=O</smiles>

S2
1) $R X, D M S O$

$\mathrm{K}_{3} \mathrm{PO}_{4}, \mathrm{rt}, 12 \mathrm{~h}$

2) TFA, DCM $\mathrm{rt}, 3 \mathrm{~h}$<smiles>[R]C1(C(=O)O)CCCCC1=O</smiles><smiles>O=C(O)C1(C(=O)O)CCCCC1=O</smiles>

1d

1-ethyl-2-oxocyclohexane-1-carboxylic acid (1d). Tert-Butyl 2-oxocyclohexane-1carboxylate $^{4}$ (990 mg, 5 mmol, purchased from Sigma-Aldrich) was dissolved in DMSO (5 mL). $\mathrm{K}_{3} \mathrm{PO}_{4}(2.1 \mathrm{~g}, 10 \mathrm{mmol})$ and $\mathrm{EtI}(2.4 \mathrm{~g}, 15 \mathrm{mmol})$ were added subsequently. The resulting mixture was allowed to stir at room temperature for $12 \mathrm{~h}$ and then diluted with a mixture of ether and hexanes $(25 \mathrm{~mL} / 25 \mathrm{~mL})$. The organic mixture was washed with brine $(2 \times 25 \mathrm{~mL})$, dried over $\mathrm{Na}_{2} \mathrm{SO}_{4}$ and concentrated under reduced pressure to give the crude product which was directly submitted to next step without further purification.

The crude product obtained from above was dissolved in DCM $(10 \mathrm{~mL})$ and cooled to 0 ${ }^{\circ} \mathrm{C}$ in an ice bath. TFA $(5 \mathrm{~mL})$ was added and the reaction mixture was allowed to stir at room temperature for 3 hours and then concentrated under reduced pressure to give the crude product which was further purified by column chromatography (20\% to $50 \% \mathrm{Et}_{2} \mathrm{O} /$ hexanes gradient) to give title product in $41 \%$ yield $(349 \mathrm{mg})$ over two steps as a white solid. $\mathrm{R}_{\mathrm{f}}=0.12(50 \%$ EtOAc/hexanes, $\mathrm{KMnO}_{4}$ stain). ${ }^{1} \mathrm{H}$ NMR $\left(500 \mathrm{MHz}, \mathrm{CDCl}_{3}\right) \delta \delta 2.58-2.43(\mathrm{~m}, 2 \mathrm{H}), 2.41-2.33$ 
(m, 1H), $2.04-1.91(\mathrm{~m}, 2 \mathrm{H}), 1.83-1.69(\mathrm{~m}, 4 \mathrm{H}), 1.68-1.60(\mathrm{~m}, 1 \mathrm{H}), 0.89(\mathrm{t}, \mathrm{J}=7.5 \mathrm{~Hz}, 3 \mathrm{H})$.

${ }^{13} \mathrm{C} \mathrm{NMR}\left(125 \mathrm{MHz}, \mathrm{CDCl}_{3}\right) \delta 209.1,177.8,60.9,40.7,35.2,27.7,27.4,22.2,8.8$. HRMS (ESI)

m/z: $[\mathrm{M}+\mathrm{H}]+$ calcd for $\mathrm{C}_{9} \mathrm{H}_{14} \mathrm{O}_{3}$ 171.1016; Found 171.1025 .

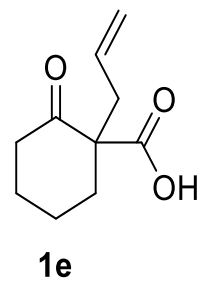

1-allyl-2-oxocyclohexane-1-carboxylic acid (1e). Tert-Butyl 2-oxocyclohexane-1carboxylate $^{4}$ (990 mg, 5 mmol, purchased from Sigma-Aldrich) was dissolved in DMSO (5 mL). $\mathrm{K}_{3} \mathrm{PO}_{4}(2.1 \mathrm{~g}, 10 \mathrm{mmol})$ and allyl bromide $(1.8 \mathrm{~g}, 15 \mathrm{mmol})$ were added subsequently. The resulting mixture was allowed to stir at room temperature for $12 \mathrm{~h}$ and then diluted with a mixture of ether and hexanes $(25 \mathrm{~mL} / 25 \mathrm{~mL})$. The organic mixture was washed with brine $(2 \times 25 \mathrm{~mL})$, dried over $\mathrm{Na}_{2} \mathrm{SO}_{4}$ and concentrated under reduced pressure to give the crude product which was directly submitted to next step without further purification.

The crude product obtained from above was dissolved in DCM $(10 \mathrm{~mL})$ and cooled to 0 ${ }^{\circ} \mathrm{C}$ in an ice bath. TFA $(5 \mathrm{~mL})$ was added and the reaction mixture was allowed to stir at room temperature for 3 hours and then concentrated under reduced pressure to give the crude product which was further purified by column chromatography (20\% to $50 \% \mathrm{Et}_{2} \mathrm{O} /$ hexanes gradient) to give title product in $47 \%$ yield $(428 \mathrm{mg})$ over two steps as a white solid. $\mathrm{R}_{\mathrm{f}}=0.15(50 \%$ EtOAc/hexanes, $\mathrm{KMnO}_{4}$ stain). ${ }^{1} \mathrm{H}$ NMR $\left(500 \mathrm{MHz} \mathrm{CDCl}_{3}\right) \delta 6.27$ (ddt, $J=17.4,10.3,7.4 \mathrm{~Hz}$, 1H), $5.73-5.51(\mathrm{~m}, 2 \mathrm{H}), 3.17(\mathrm{ddt}, J=14.0,7.0,1.2 \mathrm{~Hz}, 1 \mathrm{H}), 3.13-2.86(\mathrm{~m}, 4 \mathrm{H}), 2.52(\mathrm{~m}, 1 \mathrm{H})$, $2.40-2.04(\mathrm{~m}, 4 \mathrm{H}) .{ }^{13} \mathrm{C} \mathrm{NMR}\left(125 \mathrm{MHz}, \mathrm{CDCl}_{3}\right) \delta 207.9,177.4,132.7,118.8,60.7,40.8,39.2$ 35.4, 27.3, 22.2. HRMS (ESI) m/z: $[\mathrm{M}+\mathrm{H}]+$ calcd for $\mathrm{C}_{10} \mathrm{H}_{14} \mathrm{O}_{3}$ 183.1016; Found 183.1022. 


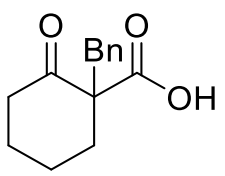

$1 \mathrm{~g}$

1-benzyl-2-oxocyclohexane-1-carboxylic acid (1g). Synthesized via a reported procedure in $57 \%$ yield as a white solid. The ${ }^{1} \mathrm{H}$ NMR and ${ }^{13} \mathrm{C}$ NMR spectra matched with reported data. ${ }^{2}$

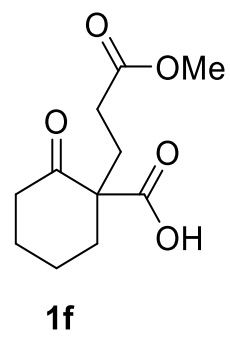

1-(3-methoxy-3-oxopropyl)-2-oxocyclohexane-1-carboxylic acid (1f). Tert-Butyl 2oxocyclohexane-1-carboxylate ${ }^{4}$ (990 mg, $5 \mathrm{mmol}$, purchased from Sigma-Aldrich) was dissolved in mixture of tert-butanol and DMSO $(1 \mathrm{~mL} / 5 \mathrm{~mL}) \cdot t \mathrm{BuOK}(112 \mathrm{mg}, 1.0 \mathrm{mmol})$ and methyl acrylate $(860 \mathrm{mg}, 10 \mathrm{mmol})$ were added subsequently. The resulting mixture was allowed to stir at $60{ }^{\circ} \mathrm{C}$ for $12 \mathrm{~h}$ and then diluted with a mixture of ether and hexanes $(25 \mathrm{~mL} / 25 \mathrm{~mL})$. The organic mixture was washed with brine $(2 \times 25 \mathrm{~mL})$, dried over $\mathrm{Na}_{2} \mathrm{SO}_{4}$ and concentrated under reduced pressure to give the crude product which was directly submitted to next step without further purification.

The crude product obtained from above was dissolved in DCM $(10 \mathrm{~mL})$ and cooled to 0 ${ }^{\circ} \mathrm{C}$ in an ice bath. TFA $(5 \mathrm{~mL})$ was added and the reaction mixture was allowed to stir at room temperature for 3 hours and then concentrated under reduced pressure to give the crude product which was further purified by column chromatography (20\% to $50 \% \mathrm{Et}_{2} \mathrm{O} /$ hexanes gradient) to give title product in $43 \%$ yield $(490 \mathrm{mg})$ over two steps as a white solid. $\mathrm{R}_{\mathrm{f}}=0.10(50 \%$ EtOAc/hexanes, $\mathrm{KMnO}_{4}$ stain). ${ }^{1} \mathrm{H}$ NMR $\left(500 \mathrm{MHz}, \mathrm{CDCl}_{3}\right) \delta 3.56$ (s, 3H), 2.49 (td, $J=12.7$, 
12.1, $5.8 \mathrm{~Hz}, 1 \mathrm{H}), 2.37$ (ddt, $J=14.2,9.0,4.6 \mathrm{~Hz}, 2 \mathrm{H}), 2.25(\mathrm{ddt}, J=16.3,10.8,5.5 \mathrm{~Hz}, 1 \mathrm{H}), 2.11$ $(\mathrm{ddd}, J=15.8,10.5,5.4 \mathrm{~Hz}, 1 \mathrm{H}), 2.07-1.79(\mathrm{~m}, 3 \mathrm{H}), 1.80-1.52(\mathrm{~m}, 3 \mathrm{H}), 1.45(\mathrm{ddd}, J=15.0$ 11.5, 4.3 Hz, 1H). ${ }^{13} \mathrm{C} \mathrm{NMR}\left(125 \mathrm{MHz}, \mathrm{CDCl}_{3}\right) \delta 208.0,176.2,173.8,59.7,51.7,40.7,36.0,29.4$, 29.3, 27.3, 22.2. HRMS (ESI) m/z: [M+H]+ calcd for $\mathrm{C}_{11} \mathrm{H}_{16} \mathrm{O}_{5}$ 229.1017; Found 229.1010.

\section{Synthesis of Allyl Carbonates}

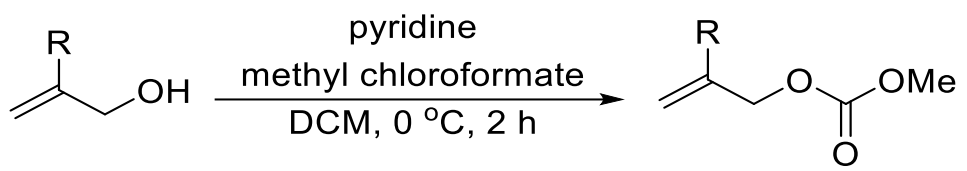

Common procedure I: Methyl chloroformate $(940 \mathrm{mg}, 10 \mathrm{mmol})$ was added to a solution of the corresponding alcohol $(5 \mathrm{mmol})$ and pyridine $(1.20 \mathrm{~g}, 15 \mathrm{mmol})$ in $20 \mathrm{~mL} \mathrm{DCM}$ at $0{ }^{\circ} \mathrm{C}$ via syringe. The mixture was stirred at $0{ }^{\circ} \mathrm{C}$ for 1 hour and then warmed up to room temperature and then concentrated under reduced pressure to give the crude product, which was further purified via column chromatography.

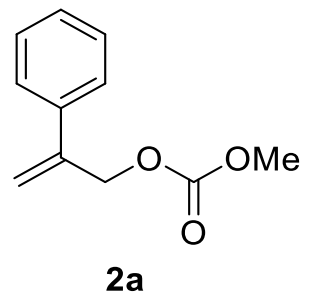

Methyl (2-phenylallyl) carbonate (2a). Synthesized via common procedure I in $85 \%$ yield as a colorless liquid. The ${ }^{1} \mathrm{H}$ NMR and ${ }^{13} \mathrm{C}$ NMR spectra matched with reported data. ${ }^{5}$<smiles>C=C(COC(=O)OC)c1ccc(F)cc1</smiles> 
2-(4-fluorophenyl)allyl methyl carbonate (2b). Synthesized via common procedure I in 95\% yield as a colorless liquid. $\mathrm{R}_{\mathrm{f}}=0.68\left(20 \%\right.$ EtOAc/hexanes, UV-Vis). ${ }^{1} \mathrm{H} \mathrm{NMR}(500 \mathrm{MHz}$, $\left.\mathrm{CDCl}_{3}\right) \delta 7.37(\mathrm{~m}, 2 \mathrm{H}), 7.00(\mathrm{~m}, 2 \mathrm{H}), 5.47(\mathrm{~s}, 1 \mathrm{H}), 5.36(\mathrm{~m}, 1 \mathrm{H}), 4.97(\mathrm{~m}, 2 \mathrm{H}), 3.73(\mathrm{~s}, 3 \mathrm{H}) .{ }^{13} \mathrm{C}$ NMR $\left(125 \mathrm{MHz}, \mathrm{CDCl}_{3}\right) \delta 162.6\left(\mathrm{~d}, J_{F}=246.2 \mathrm{~Hz}\right), 155.5,141.2,133.8\left(\mathrm{~d}, J_{F}=3.8 \mathrm{~Hz}\right), 127.7$ $\left(\mathrm{d}, J_{F}=7.5 \mathrm{~Hz}\right), 115.5\left(\mathrm{~d}, J_{F}=1.3 \mathrm{~Hz}\right), 115.3\left(\mathrm{~d}, J_{F}=21.2 \mathrm{~Hz}\right), 68.9,54.7 .{ }^{19} \mathrm{~F}$ NMR $(300 \mathrm{MHz}$, $\left.\mathrm{CDCl}_{3}\right) \delta$-114.0. HRMS (ESI) m/z: [M+H]+ calcd for $\mathrm{C}_{11} \mathrm{H}_{11} \mathrm{FO}_{3}$ 211.0765; Found 211.0787.

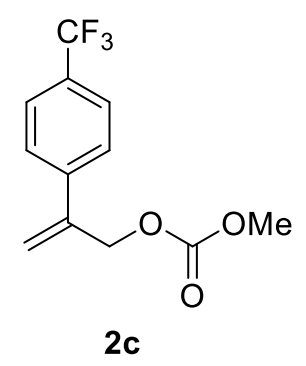

methyl (2-(4-(trifluoromethyl)phenyl)allyl) carbonate (2c). Synthesized via common procedure I in $82 \%$ yield as a colorless liquid. $\mathrm{R}_{\mathrm{f}}=0.69\left(20 \%\right.$ EtOAc/hexanes, UV-Vis). ${ }^{1} \mathrm{H}$ NMR $\left(500 \mathrm{MHz}, \mathrm{CDCl}_{3}\right) \delta 7.58(\mathrm{~m}, 2 \mathrm{H}), 7.52(\mathrm{~m}, 2 \mathrm{H}), 5.62(\mathrm{~s}, 1 \mathrm{H}), 5.50(\mathrm{t}, J=1.3 \mathrm{~Hz}, 1 \mathrm{H}), 5.03(\mathrm{~d}, J$ $=1.3 \mathrm{~Hz}, 2 \mathrm{H}), 3.76(\mathrm{~s}, 3 \mathrm{H}) .{ }^{13} \mathrm{C} \mathrm{NMR}\left(125 \mathrm{MHz}, \mathrm{CDCl}_{3}\right) \delta 155.5,141.3,141.2,130.0\left(\mathrm{q}, J_{F}=\right.$ $32.5 \mathrm{~Hz}), 126.3,125.4\left(\mathrm{q}, J_{F}=3.8 \mathrm{~Hz}\right), 124.1\left(\mathrm{q}, J_{F}=260 \mathrm{~Hz}\right), 117.7,68.6,54.7 .{ }^{19} \mathrm{~F}$ NMR $(300$ $\left.\mathrm{MHz}, \mathrm{CDCl}_{3}\right) \delta$-62.9. HRMS (ESI) $\mathrm{m} / \mathrm{z}$ : $[\mathrm{M}+\mathrm{H}]+$ calcd for $\mathrm{C}_{12} \mathrm{H}_{11} \mathrm{~F}_{3} \mathrm{O}_{3}$ 261.0733; Found 261.0739 .

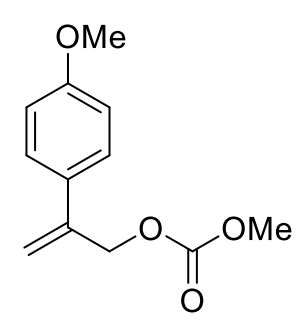

2d

2-(4-methoxyphenyl)allyl methyl carbonate (2d). Synthesized via common procedure I in $93 \%$ yield as a colorless liquid. $\mathrm{R}_{\mathrm{f}}=0.60\left(20 \%\right.$ EtOAc/hexanes, UV-Vis). ${ }^{1} \mathrm{H}$ NMR $(500 \mathrm{MHz}$, 
$\left.\mathrm{CDCl}_{3}\right) \delta 7.37(\mathrm{~m}, 2 \mathrm{H}), 6.86(\mathrm{~m}, 2 \mathrm{H}), 5.48(\mathrm{~d}, J=1.0 \mathrm{~Hz}, 1 \mathrm{H}), 5.30(\mathrm{q}, J=1.2 \mathrm{~Hz}, 1 \mathrm{H}), 5.00(\mathrm{~m}$, 2H), $3.73(\mathrm{~m}, 6 \mathrm{H}) .{ }^{13} \mathrm{C} \mathrm{NMR}\left(125 \mathrm{MHz}, \mathrm{CDCl}_{3}\right) \delta 159.6,155.6,141.5,130.1,127.1,121.8,113.9$, 69.1, 55.0, 54.7. HRMS (ESI) m/z: [M+H]+ calcd for $\mathrm{C}_{12} \mathrm{H}_{14} \mathrm{O}_{4}$ 223.0965; Found 223.0976.

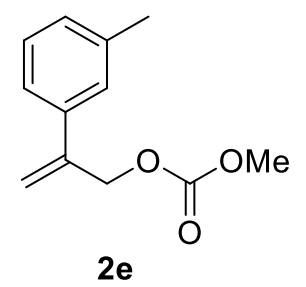

Methyl (2-(m-tolyl)allyl) carbonate (2e). Synthesized via common procedure I from corresponding alcohol in $90 \%$ yield as a colorless liquid. $R_{\mathrm{f}}=0.72(20 \%$ EtOAc/hexanes, UVVis). ${ }^{1} \mathrm{H}$ NMR (400 MHz, $\left.\mathrm{CDCl}_{3}\right) \delta 7.25-7.12(\mathrm{~m}, 4 \mathrm{H}), 5.51(\mathrm{p}, \mathrm{J}=1.6 \mathrm{~Hz}, 1 \mathrm{H}), 5.13(\mathrm{p}, \mathrm{J}=1.2$ $\mathrm{Hz}, 1 \mathrm{H}), 4.80-4.83(\mathrm{~m}, 2 \mathrm{H}), 3.79(\mathrm{~s}, 3 \mathrm{H}), 2.34(\mathrm{~s}, 3 \mathrm{H}) .{ }^{13} \mathrm{C} \mathrm{NMR}\left(125 \mathrm{MHz}, \mathrm{CDCl}_{3}\right) \delta$ 155.7, 142.2, 138.1, 137.8, 128.9, 128.4, 126.8, 123.1, 115.4, 69.1, 54.8, 21.5. HRMS (ESI) m/z: [M+H]+ calcd for $\mathrm{C}_{12} \mathrm{H}_{14} \mathrm{O}_{3}$ 207.1016; Found 207.1021.

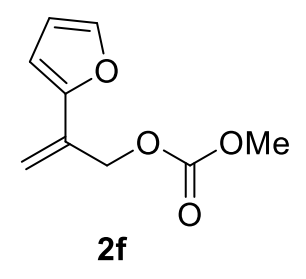

2-(furan-2-yl)allyl methyl carbonate (2f). Synthesized via common procedure I from corresponding alcohol in $90 \%$ yield as a light-yellow liquid. $\mathrm{R}_{\mathrm{f}}=0.55(20 \%$ EtOAc/hexanes, UVVis). ${ }^{1} \mathrm{H}$ NMR (300 MHz, $\left.\mathrm{CDCl}_{3}\right) \delta 7.39(\mathrm{~s}, 1 \mathrm{H}), 6.42-6.34(\mathrm{~m}, 2 \mathrm{H}), 5.73(\mathrm{~s}, 1 \mathrm{H}), 5.31(\mathrm{~s}, 1 \mathrm{H})$, $4.92(\mathrm{~s}, 2 \mathrm{H}), 3.80(\mathrm{~s}, 3 \mathrm{H}) .{ }^{13} \mathrm{C} \mathrm{NMR}\left(101 \mathrm{MHz}, \mathrm{CDCl}_{3}\right) \delta$ 155.6, 151.7, 142.4, 131.9, 113.0, 111.3, 107.1, 67.6, 54.9. HRMS (ESI) m/z: [M+H]+ calcd for $\mathrm{C}_{9} \mathrm{H}_{10} \mathrm{O}_{4}$ 183.0652; Found 183.0655. 


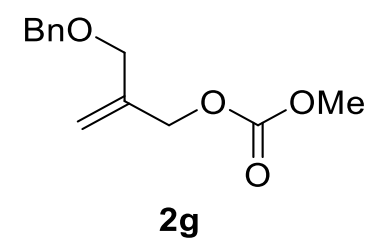

2-((benzyloxy)methyl)allyl methyl carbonate (2g). Synthesized via common procedure I from corresponding alcohol in $90 \%$ yield as a colorless liquid. ${ }^{1} \mathrm{H} \mathrm{NMR}\left(500 \mathrm{MHz}, \mathrm{CDCl}_{3}\right) \delta$ $7.33-7.19(\mathrm{~m}, 5 \mathrm{H}), 5.24-5.21(\mathrm{~m}, 2 \mathrm{H}), 4.64(\mathrm{~s}, 2 \mathrm{H}), 4.45(\mathrm{~s}, 2 \mathrm{H}), 4.00(\mathrm{~s}, 2 \mathrm{H}), 3.72(\mathrm{~s}, 3 \mathrm{H}) .{ }^{13} \mathrm{C}$ NMR $\left(150 \mathrm{MHz}, \mathrm{CDCl}_{3}\right) \delta$ 155.6, 140.3, 138.1, 128.4, 127.7, 127.7, 115.8, 72.2, 70.6, 68.1, 54.8. HRMS (ESI) m/z: [M+H]+ calcd for $\mathrm{C}_{13} \mathrm{H}_{16} \mathrm{O}_{4} 237.1121$; Found 237.1129.

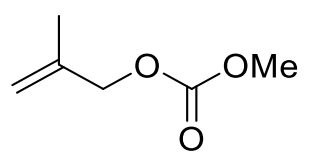

$2 \mathrm{~h}$

Methyl (2-methylallyl) carbonate (2h). Synthesized via common procedure I from corresponding alcohol in $65 \%$ yield as a colorless liquid. The ${ }^{1} \mathrm{H}$ NMR and ${ }^{13} \mathrm{C}$ NMR spectra matched with reported data. ${ }^{5}$

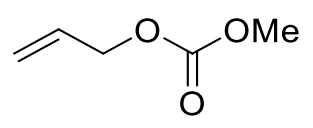

$2 \mathbf{i}$

Allyl methyl carbonate was purchased from Sigma-Aldrich.

\section{Scope Studies of the Enantioselective Intermolecular Decarboxylative Allylation}

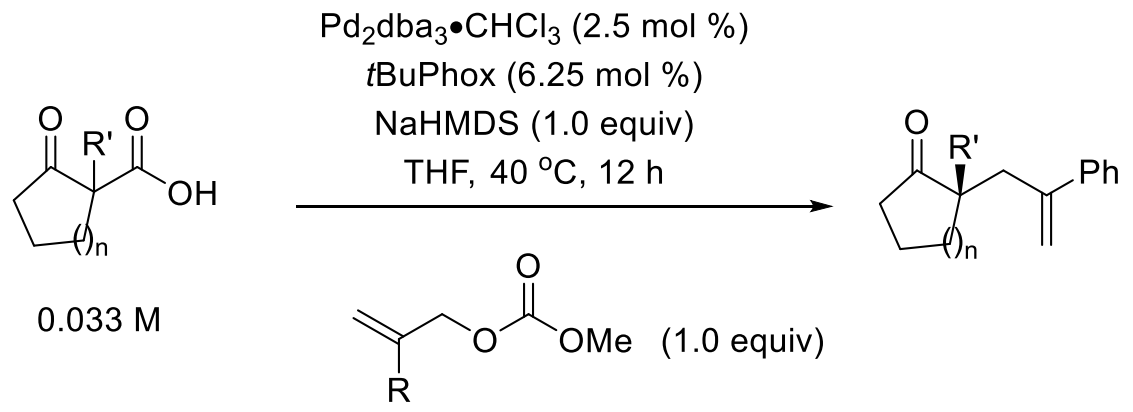


Common procedure II: In a glove box was added $\mathrm{Pd}_{2} \mathrm{dba}_{3}(5.1 \mathrm{mg}, 0.005 \mathrm{mmol}, 2.5 \mathrm{~mol} \%)$ and ligand $(S)-t$-BuPhox $(4.8 \mathrm{mg}, 0.0125 \mathrm{mmol}, 6.25 \mathrm{~mol} \%)$ to a flask containing a magnetic stirrer. In a separate flask containing a magnetic stirrer, NaHMDS (37 mg, $0.2 \mathrm{mmol}, 1$ equiv) was added. Both flasks were capped and removed from the glove box and placed under a nitrogen atmosphere. THF ( $2 \mathrm{~mL}$ ) was added to the palladium/ligand mixture and pre-stirred at room temperature for $0.5 \mathrm{~h}$. To the flask containing NaHMDS was added $2 \mathrm{~mL}$ of THF followed by transfer of carboxylic acid $(0.2 \mathrm{mmol})$ into the NaHMDS solution using a total of $2 \mathrm{~mL}$ THF for the transfer. The palladium/ligand mixture was then transferred via syringe to the flask containing the NaHMDS/carboxylic acid mixture. Lastly, allyl carbonate $(0.2 \mathrm{mmol})$ was added to the reaction mixture neat. The reaction was stirred at $40{ }^{\circ} \mathrm{C}$ for $12 \mathrm{~h}$. The solvent was evaporated, and the crude material was purified via flash column chromatography on silica gel to give desired product.

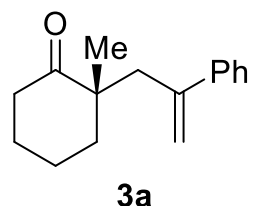

(S)-2-methyl-2-(2-phenylallyl)cyclohexan-1-one (3a). Synthesized via common procedure II employing acid 1a and allyl carbonate 2a in $86 \%$ yield $(39.2 \mathrm{mg})$ as a colorless liquid. $\mathrm{R}_{\mathrm{f}}=0.75$ (20\% EtOAc/hexanes, UV-Vis). ${ }^{1} \mathrm{H}$ NMR $\left(500 \mathrm{MHz}, \mathrm{CDCl}_{3}\right) \delta 7.43-7.21(\mathrm{~m}, 5 \mathrm{H})$, $5.27(\mathrm{~d}, J=1.7 \mathrm{~Hz}, 1 \mathrm{H}), 5.07(\mathrm{dd}, J=1.7,1.0 \mathrm{~Hz}, 1 \mathrm{H}), 2.96(\mathrm{dd}, J=13.8,1.0 \mathrm{~Hz}, 1 \mathrm{H}), 2.76(\mathrm{~d}, J$ $=13.8 \mathrm{~Hz}, 1 \mathrm{H}), 2.36(\mathrm{~m}, 2 \mathrm{H}), 1.91-1.59(\mathrm{~m}, 5 \mathrm{H}), 1.51(\mathrm{ddt}, \mathrm{J}=12.9,9.3,2.7 \mathrm{~Hz}, 1 \mathrm{H}), 0.96(\mathrm{~s}$, $3 \mathrm{H}) .{ }^{13} \mathrm{C} \mathrm{NMR}\left(125 \mathrm{MHz}, \mathrm{CDCl}_{3}\right) \delta 215.3,145.8,143.2,128.2,127.3,126.6,117.4,49.1,42.2$, 39.1, 38.8, 27.3, 23.1, 21.1. $[\alpha]^{20} \mathrm{D}=-18.4\left(\mathrm{c}=1.0, \mathrm{CHCl}_{3}\right)$. Enantiomeric excess was determined by HPLC with a Chiralcel OJ-H column (99:1, hexanes:isopropanol, $1.0 \mathrm{~mL} / \mathrm{min}, 254 \mathrm{~nm})$; minor $\operatorname{tr}=15.8$ min; major $\operatorname{tr}=18.8 \mathrm{~min} ; 92 \%$ ee. HRMS $(\mathrm{ESI}) \mathrm{m} / \mathrm{z}:[\mathrm{M}+\mathrm{H}]+$ calcd for $\mathrm{C}_{16} \mathrm{H}_{20} \mathrm{O}$ 229.1587; Found 229.1596. 

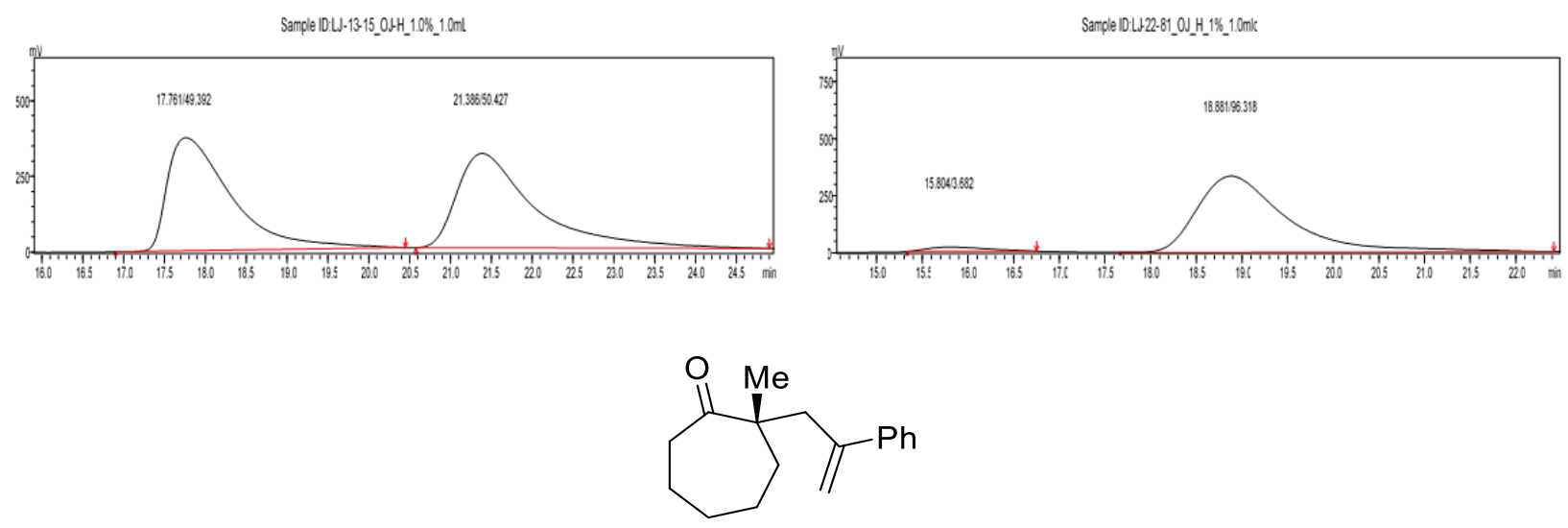

3b

(S)-2-methyl-2-(2-phenylallyl)cycloheptan-1-one (3b). Synthesized via common procedure II employing acid $\mathbf{1 b}$ and allyl carbonate 2a in $80 \%$ yield $(38.8 \mathrm{mg}$ ) as a colorless liquid. $\mathrm{R}_{\mathrm{f}}=0.74\left(20 \%\right.$ EtOAc/hexanes, UV-Vis). ${ }^{1} \mathrm{H}$ NMR (500 MHz, $\left.\mathrm{CDCl}_{3}\right) \delta 7.32(\mathrm{~m}, 5 \mathrm{H}), 5.29(\mathrm{~m}$, $1 \mathrm{H}), 5.05(\mathrm{~d}, J=1.3 \mathrm{~Hz}, 1 \mathrm{H}), 2.76(\mathrm{~m}, 2 \mathrm{H}), 2.51(\mathrm{~m}, 1 \mathrm{H}), 2.20(\mathrm{dd}, \mathrm{J}=14.9,5.8 \mathrm{~Hz}, 1 \mathrm{H}), 1.75-$ $1.36(\mathrm{~m}, 8 \mathrm{H}), 0.96(\mathrm{~s}, 3 \mathrm{H}) .{ }^{13} \mathrm{C} \mathrm{NMR}\left(125 \mathrm{MHz}, \mathrm{CDCl}_{3}\right) \delta 217.5,145.7,142.5,128.2,127.4,126.6$ 117.4, 51.4, 44.3, 40.7, 36.8, 30.6, 26.6, 24.3, 23.2. $[\alpha]^{22} \mathrm{D}=-22.4\left(\mathrm{c}=1.0, \mathrm{CHCl}_{3}\right)$. Enantiomeric excess was determined by HPLC with a Chiralcel OJ-H column (99:1, hexanes:isopropanol, 1.0 $\mathrm{mL} / \mathrm{min}, 254 \mathrm{~nm})$; $\operatorname{minor} \operatorname{tr}=13.6 \mathrm{~min}$; major $\operatorname{tr}=18.6 \mathrm{~min} ; 91 \%$ ee. HRMS $(\mathrm{ESI}) \mathrm{m} / \mathrm{z}:[\mathrm{M}+\mathrm{H}]+$ calcd for $\mathrm{C}_{17} \mathrm{H}_{22} \mathrm{O} 243.1743$; Found 243.1748.
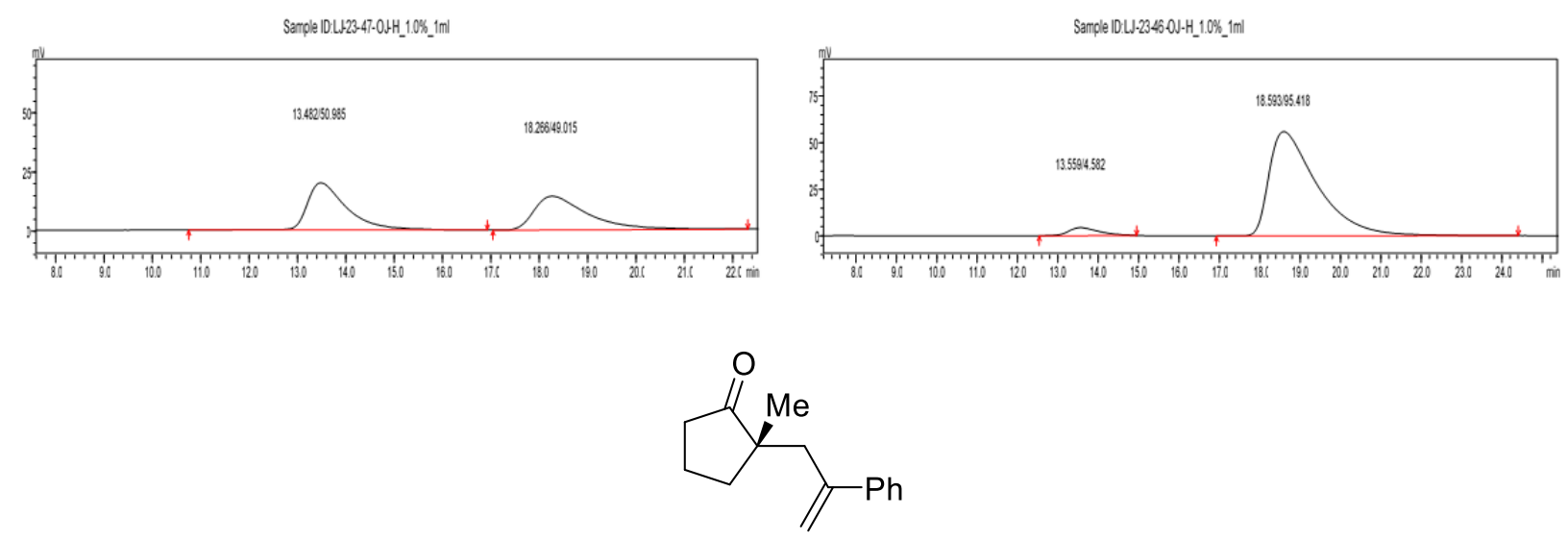

$3 c$ 
(S)-2-methyl-2-(2-phenylallyl)cyclopentan-1-one (3c). Condition A: $\mathrm{Pd}_{2} \mathrm{dbaa}_{3} \cdot \mathrm{CHCl}_{3}$ (5.1 mg, $0.005 \mathrm{mmol}),(S)-t$ Bu-Phox (4.8 mg, $0.0125 \mathrm{mmol})$ and NaHMDS (37 mg, $0.2 \mathrm{mmol})$ were weighed out in an oven-dried round bottom flask in a glove box. The flask was sealed with a septum, taken out and connected with an argon balloon via a needle. THF $(2.0 \mathrm{~mL})$ was added and the resulting solution was allowed to stir at r.t. for $30 \mathrm{~min}$. Carboxylic acid 1c $(28.5 \mathrm{mg}, 0.2 \mathrm{mmol}$ in $2 \mathrm{~mL}$ THF) and allyl carbonate $\mathbf{2 a}$ ( $40 \mathrm{mg}, 0.2 \mathrm{mmol}$ in $2 \mathrm{~mL}$ THF) were added to the pre-stirred catalyst solution via syringe. After stirring under argon at $40{ }^{\circ} \mathrm{C}$. for $12 \mathrm{~h}$, the reaction mixture was adsorbed onto silica and purified by column chromatography to give desired product in $75 \%$ yield $(31.5 \mathrm{mg})$ as a colorless oil. Enantiomeric excess was determined by HPLC with a Chiralpak AsHH column (99.7:0.3, hexanes:isopropanol, $1.0 \mathrm{~mL} / \mathrm{min}, 254 \mathrm{~nm})$; minor tr $=14.9$ min; major $\operatorname{tr}=$ $31.7 \mathrm{~min} ; 80 \%$ ee.
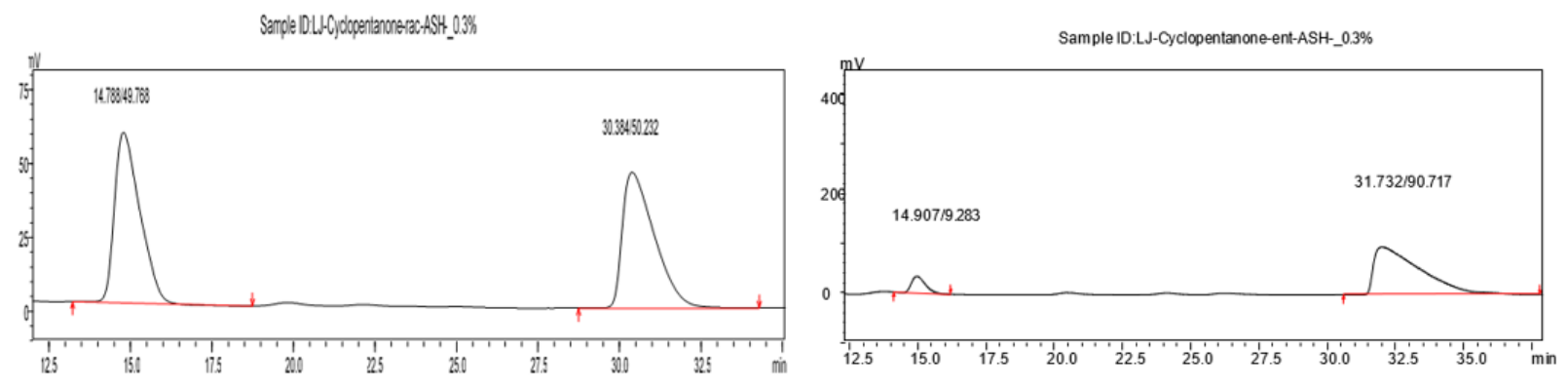

Condition B: $\mathrm{Pd}_{2} \mathrm{dba}_{3} \cdot \mathrm{CHCl}_{3}(5.1 \mathrm{mg}, 0.005 \mathrm{mmol}),(S)-t \mathrm{Bu}-\left(\mathrm{CF}_{3}\right)_{3} \mathrm{Phox}(7.8 \mathrm{mg}, 0.0125$ mmol) and NaHMDS (37 mg, $0.2 \mathrm{mmol}$ ) were weighed out in an oven-dried round bottom flask in a glove box. The flask was sealed with a septum, taken out and connected with an argon balloon via a needle. THF $(2.0 \mathrm{~mL})$ was added, and the resulting solution was allowed to stir at r.t. for 30 min. Carboxylic acid 1c (28.5 mg, $0.2 \mathrm{mmol}$ in $2 \mathrm{~mL}$ THF) and allyl carbonate $2 \mathbf{a}(40 \mathrm{mg}, 0.2$ $\mathrm{mmol}$ in $2 \mathrm{~mL}$ THF) were added to the pre-stirred catalyst solution via syringe. After stirring under 
argon at $40{ }^{\circ} \mathrm{C}$. for $12 \mathrm{~h}$, the reaction mixture was adsorbed onto silica and purified by column chromatography to give desired product in $72 \%$ yield $(30.2 \mathrm{mg})$ as a colorless oil. $\mathrm{R}_{\mathrm{f}}=0.70(20 \%$ EtOAc/hexanes, UV-Vis). ${ }^{1} \mathrm{H}$ NMR $\left(500 \mathrm{MHz}, \mathrm{CDCl}_{3}\right) \delta 7.43-7.27(\mathrm{~m}, 5 \mathrm{H}), 5.30(\mathrm{~d}, \mathrm{~J}=1.7 \mathrm{~Hz}$, 1H), $5.17-5.01(\mathrm{~m}, 1 \mathrm{H}), 2.79-2.73(\mathrm{~m}, 1 \mathrm{H}), 2.65(\mathrm{~d}, J=13.8 \mathrm{~Hz}, 1 \mathrm{H}), 2.30-2.19(\mathrm{~m}, 1 \mathrm{H}), 2.08$ $-1.93(\mathrm{~m}, 1 \mathrm{H}), 1.90-1.65(\mathrm{~m}, 3 \mathrm{H}), 1.54-1.41(\mathrm{~m}, 1 \mathrm{H}), 0.94(\mathrm{~s}, 3 \mathrm{H}) .{ }^{13} \mathrm{C}$ NMR $(125 \mathrm{MHz}$ $\left.\mathrm{CDCl}_{3}\right) \delta 223.1,146.0,142.3,128.2,127.4,126.7,117.1,48.9,41.7,37.4,35.2,22.5,18.6 .[\alpha]^{22} \mathrm{D}$ $=-60.1\left(\mathrm{c}=1.0, \mathrm{CHCl}_{3}\right)$. Enantiomeric excess was determined by HPLC with a Chiralpak AsH-H column (99.7:0.3, hexanes:isopropanol, $1.0 \mathrm{~mL} / \mathrm{min}, 254 \mathrm{~nm})$; minor $\operatorname{tr}=14.8$ min; major $\operatorname{tr}=29.3$ min; 90\% ee. HRMS (ESI) m/z: [M+H]+ calcd for $\mathrm{C}_{15} \mathrm{H}_{18} \mathrm{O} 215.1430$; Found 215.1420.
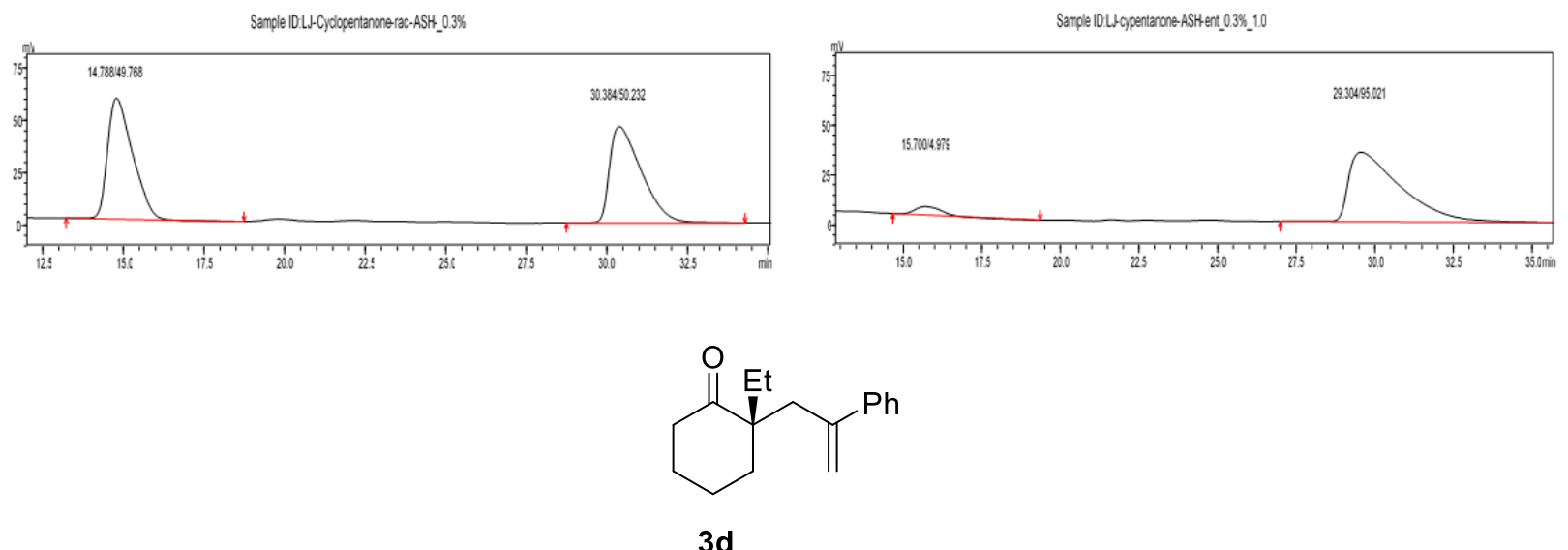

(S)-2-ethyl-2-(2-phenylallyl)cyclohexan-1-one (3d). Synthesized via common procedure II employing acid $\mathbf{1 d}$ and allyl carbonate $\mathbf{2 a}$ in $81 \%$ yield $(39.2 \mathrm{mg})$ as a colorless liquid. $\mathbf{R}_{\mathrm{f}}=0.76$ (20\% EtOAc/hexanes, UV-Vis). ${ }^{1} \mathrm{H}$ NMR (500 MHz, $\left.\mathrm{CDCl}_{3}\right) \delta 7.45-7.16(\mathrm{~m}, 5 \mathrm{H}), 5.25(\mathrm{~d}, \mathrm{~J}=$ $1.8 \mathrm{~Hz}, 1 \mathrm{H}), 5.07(\mathrm{dd}, \mathrm{J}=1.9,1.0 \mathrm{~Hz}, 1 \mathrm{H}), 2.95(\mathrm{~d}, J=14.2 \mathrm{~Hz}, 1 \mathrm{H}), 2.72(\mathrm{~d}, J=14.1 \mathrm{~Hz}, 1 \mathrm{H})$, $2.39-2.18(\mathrm{~m}, 2 \mathrm{H}), 1.84(\mathrm{ddt}, J=15.9,7.5,5.4 \mathrm{~Hz}, 1 \mathrm{H}), 1.71-1.39(\mathrm{~m}, 7 \mathrm{H}), 0.68(\mathrm{t}, J=7.5 \mathrm{~Hz}$ 3H). ${ }^{13} \mathrm{C} \mathrm{NMR}\left(125 \mathrm{MHz}, \mathrm{CDCl}_{3}\right) \delta 215.0,146.2,143.6,128.1,127.1,126.6,117.6,52.5,39.4$, 38.6, 36.9, 27.0, 26.6, 20.9, 8.1. $[\alpha]^{22} \mathrm{D}=+1.8\left(\mathrm{c}=1.0, \mathrm{CHCl}_{3}\right)$. Enantiomeric excess was determined by SFC with a ChiralpakAD-H column (90:10, $\mathrm{CO}_{2}$ :isopropanol, $2.0 \mathrm{~mL} / \mathrm{min}, 40^{\circ} \mathrm{C}$. 
$254 \mathrm{~nm})$; minor $\operatorname{tr}=3.0 \mathrm{~min}$; major $\operatorname{tr}=3.25 \mathrm{~min} ; 96 \%$ ee. $\mathrm{HRMS}(\mathrm{ESI}) \mathrm{m} / \mathrm{z}:\left[\mathrm{M}+\mathrm{NH}_{4}\right]+$ calcd for $\mathrm{C}_{17} \mathrm{H}_{26} \mathrm{NO} 260.2009 ;$ Found 260.2012.
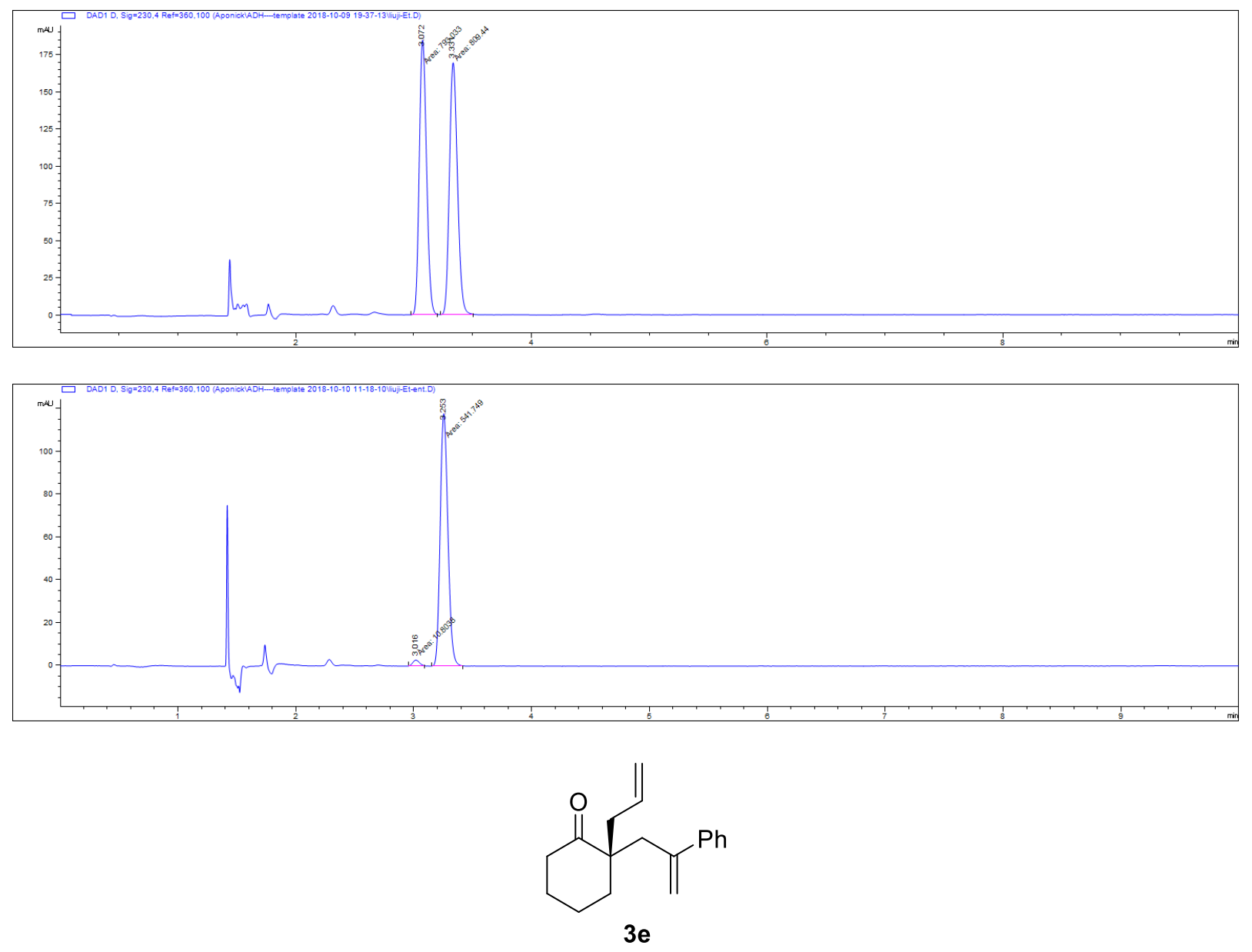

(R)-2-allyl-2-(2-phenylallyl)cyclohexan-1-one (3e). Synthesized via common procedure II employing acid $\mathbf{1 e}$ and allyl carbonate $\mathbf{2 a}$ in $70 \%$ yield $(35 \mathrm{mg})$ as a colorless liquid. $\mathrm{R}_{\mathrm{f}}=0.77$ (20\% EtOAc/hexanes, UV-Vis). ${ }^{1} \mathrm{H}$ NMR (400 MHz, $\left.\mathrm{CDCl}_{3}\right) \delta 7.29-7.18(\mathrm{~m}, 5 \mathrm{H}), 5.59-5.49$ $(\mathrm{m}, 1 \mathrm{H}), 5.20(\mathrm{~d}, \mathrm{~J}=1.7 \mathrm{~Hz}, 1 \mathrm{H}), 5.04-5.02(\mathrm{~m}, 1 \mathrm{H}), 4.97-4.93(\mathrm{~m}, 1.0 \mathrm{~Hz}, 1 \mathrm{H}), 4.87(\mathrm{dq}, \mathrm{J}=$ 17.0, 1.6 Hz, 1H), $2.89(\mathrm{dd}, \mathrm{J}=14.1,0.9 \mathrm{~Hz}, 1 \mathrm{H}), 2.70(\mathrm{dd}, \mathrm{J}=14.1,0.9 \mathrm{~Hz}, 1 \mathrm{H}), 2.27-2.16(\mathrm{~m}$, $4 \mathrm{H}), 1.74-1.66(\mathrm{~m}, \mathrm{~J}=9.7,2 \mathrm{H}), 1.65-1.58(\mathrm{~m}, 2 \mathrm{H}), 1.55-1.50(\mathrm{~m}, 2 \mathrm{H}) .{ }^{13} \mathrm{C} \mathrm{NMR}(100 \mathrm{MHz}$, $\left.\mathrm{CDCl}_{3}\right) \delta 214.2,145.9,143.4,134.0,128.3,127.4,126.8,118.1,117.9,52.4,39.9,39.7,39.2,36.6$ 27.0, 21.0. $[\alpha]^{21.3} \mathrm{D}=-5.05\left(\mathrm{c}=1.0, \mathrm{CH}_{2} \mathrm{Cl}_{2}\right)$. Enantiomeric excess was determined by HPLC with 
a Chiralpak IC-3 column (99.5:0.5, hexanes:isopropanol, $1.0 \mathrm{~mL} / \mathrm{min}, 254 \mathrm{~nm})$; minor $\mathrm{t}_{\mathrm{r}}=10.1$ min; major $\mathrm{t}_{\mathrm{r}}=10.7 \mathrm{~min}$; $95 \%$ ee. HRMS $(\mathrm{ESI}) \mathrm{m} / \mathrm{z}$ : $[\mathrm{M}+\mathrm{H}]+$ calcd for $\mathrm{C}_{18} \mathrm{H}_{22} \mathrm{O} 255.1743$; Found 255.1751 .
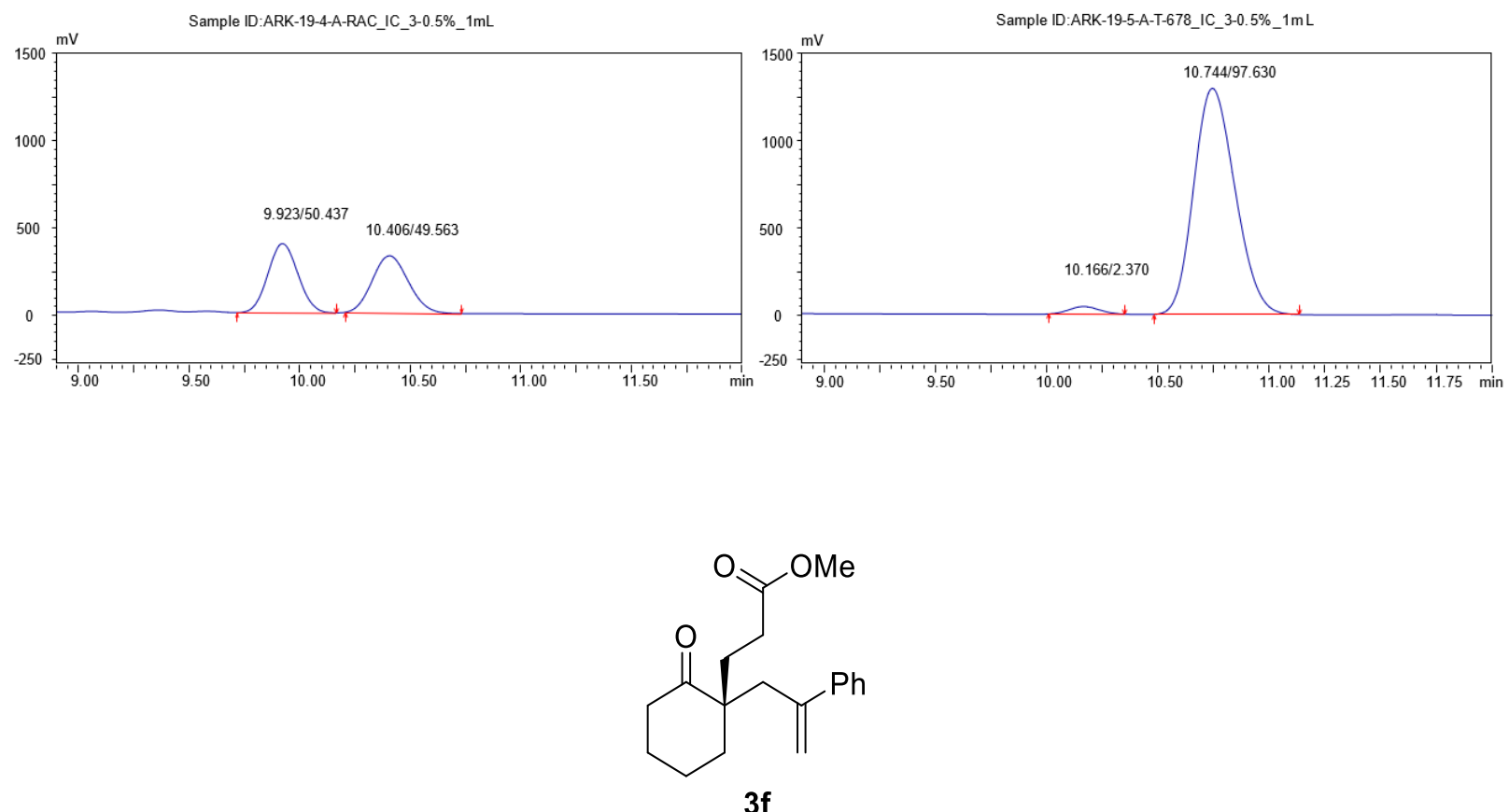

Methyl (R)-3-(2-oxo-1-(2-phenylallyl)cyclohexyl)propanoate (3f). Synthesized via common procedure II employing acid $\mathbf{1 f}$ and allyl carbonate $\mathbf{2 a}$ in $81 \%$ yield $(48.0 \mathrm{mg})$ as a colorless liquid. $\mathrm{R}_{\mathrm{f}}=0.36\left(20 \%\right.$ EtOAc/hexanes, UV-Vis). ${ }^{1} \mathrm{H}$ NMR $\left(500 \mathrm{MHz}, \mathrm{CDCl}_{3}\right) \delta 7.36-$ $7.27(\mathrm{~m}, 5 \mathrm{H}), 5.26(\mathrm{~d}, J=1.6 \mathrm{~Hz}, 1 \mathrm{H}), 5.08(\mathrm{dd}, J=1.7,0.9 \mathrm{~Hz}, 1 \mathrm{H}), 3.61(\mathrm{~s}, 3 \mathrm{H}), 2.95(\mathrm{dd}, J=$ 14.2, $0.9 \mathrm{~Hz}, 1 \mathrm{H}), 2.72(\mathrm{~d}, J=14.3 \mathrm{~Hz}, 1 \mathrm{H}), 2.33(\mathrm{~m}, 1 \mathrm{H}), 2.26-2.18(\mathrm{~m}, 1 \mathrm{H}), 2.02-1.94(\mathrm{~m}$, 2H), $1.78-1.55(\mathrm{~m}, 7 \mathrm{H}), 1.32-1.16(\mathrm{~m}, 1 \mathrm{H}) .{ }^{13} \mathrm{C} \mathrm{NMR}\left(125 \mathrm{MHz}, \mathrm{CDCl}_{3}\right) \delta 214.2,173.7,145.6$, $143.2,128.2,127.3,126.6,118.0,51.6,51.6,39.3,39.3,36.9,29.1,28.7,26.9,20.8 .[\alpha]^{20}{ }_{D}=+7.2$ $\left(\mathrm{c}=1.0, \mathrm{CHCl}_{3}\right)$. Enantiomeric excess was determined by HPLC with a Chiralpak As-H column (99.7:0.3, hexanes:isopropanol, $1.0 \mathrm{~mL} / \mathrm{min}, 254 \mathrm{~nm})$; minor $\operatorname{tr}=17.5 \mathrm{~min}$; major $\mathrm{tr}=22.6 \mathrm{~min}$; 80\% ee. HRMS (ESI) m/z: [M+H]+ calcd for $\mathrm{C}_{19} \mathrm{H}_{24} \mathrm{O}_{3} 301.2064$; Found 301.2068. 

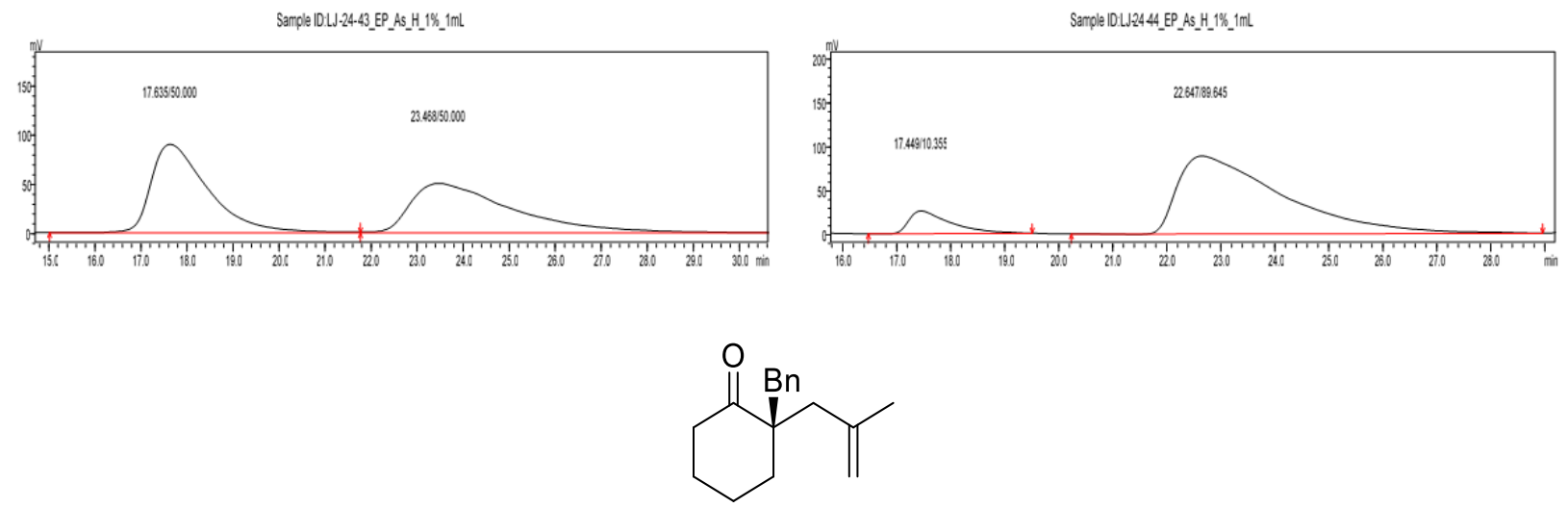

$3 \mathrm{~g}$

(S)-2-benzyl-2-(2-methylallyl)cyclohexan-1-one (3g). Synthesized via common procedure II employing acid $\mathbf{1 g}$ and allyl carbonate $\mathbf{2 h}$ in $65 \%$ yield $(31.5 \mathrm{mg}$ ) as a colorless liquid. $\mathrm{R}_{\mathrm{f}}=0.70$ (20\% EtOAc/hexanes, UV-Vis). ${ }^{1} \mathrm{H}$ NMR $\left(500 \mathrm{MHz}, \mathrm{CDCl}_{3}\right) \delta 7.33-7.02(\mathrm{~m}, 5 \mathrm{H})$, $4.89(\mathrm{~d}, J=1.5 \mathrm{~Hz}, 1 \mathrm{H}), 4.72(\mathrm{dd}, J=2.3,1.2 \mathrm{~Hz}, 1 \mathrm{H}), 3.07(\mathrm{~d}, J=13.7 \mathrm{~Hz}, 1 \mathrm{H}), 2.84(\mathrm{~d}, J=13.7$ $\mathrm{Hz}, 1 \mathrm{H}), 2.56(\mathrm{ddd}, J=14.8,9.2,5.5 \mathrm{~Hz}, 1 \mathrm{H}), 2.46-2.35(\mathrm{~m}, 3 \mathrm{H}), 1.94-1.58(\mathrm{~m}, 9 \mathrm{H}) .{ }^{13} \mathrm{C} \mathrm{NMR}$ $\left(125 \mathrm{MHz}, \mathrm{CDCl}_{3}\right) \delta 214.7,142.2,137.9,130.9,127.9,126.2,114.7,52.5,43.3,41.8,39.7,35.7$, 26.7, 24.6, 20.9. $[\alpha]^{22}=+2.00\left(\mathrm{c}=1.0, \mathrm{CHCl}_{3}\right)$. Enantiomeric excess was determined by HPLC with a ChiralpakAs-H column (99.7:0.3, hexanes:isopropanol, $1.0 \mathrm{~mL} / \mathrm{min}, 254 \mathrm{~nm})$; $\operatorname{minor} \operatorname{tr}=$ $9.7 \mathrm{~min}$; major $\operatorname{tr}=7.9 \mathrm{~min} ; 87 \%$ ee. $\mathrm{HRMS}(\mathrm{ESI}) \mathrm{m} / \mathrm{z}$ : $[\mathrm{M}+\mathrm{H}]+$ calcd for $\mathrm{C}_{17} \mathrm{H}_{22} \mathrm{O} 243.1743$; Found 243.1751.
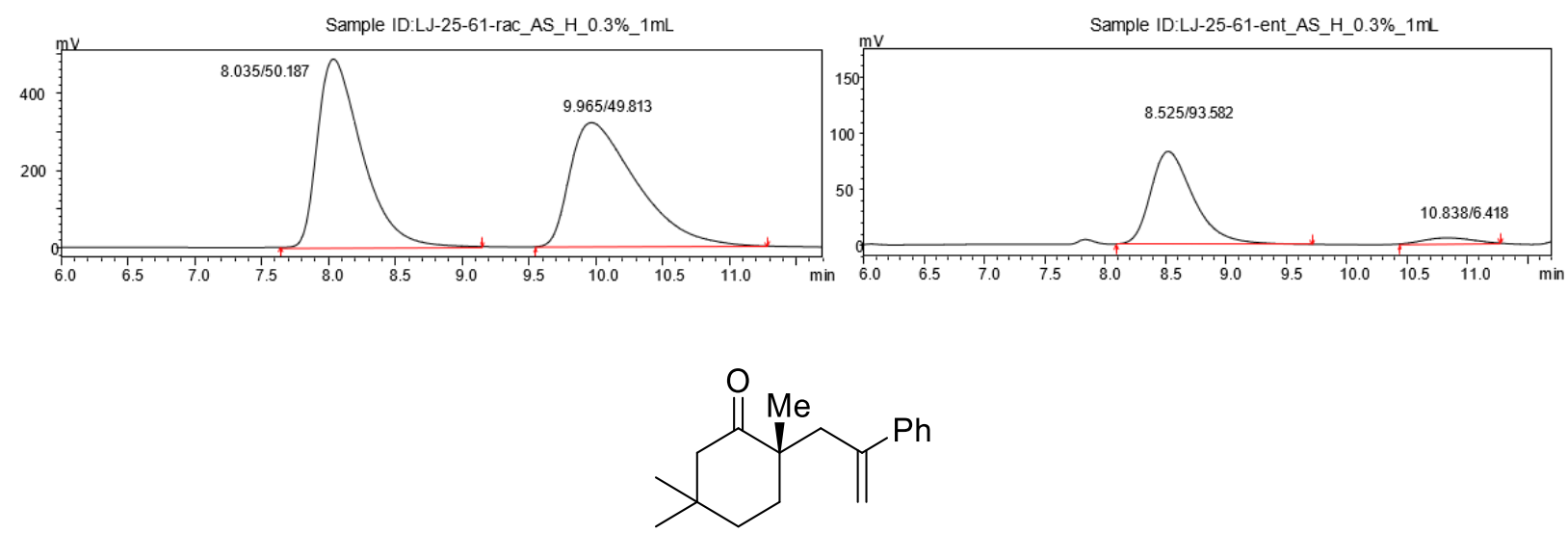
(S)-2,5,5-trimethyl-2-(2-phenylallyl)cyclohexan-1-one (3h). Synthesized via common procedure II employing acid $\mathbf{1} \mathbf{h}$ and allyl carbonate $\mathbf{2 a}$ in $85 \%$ yield $(43.5 \mathrm{mg})$ as a colorless liquid. $\mathrm{R}_{\mathrm{f}}=0.77\left(20 \%\right.$ EtOAc/hexanes, UV-Vis). ${ }^{1} \mathrm{H} \mathrm{NMR}\left(500 \mathrm{MHz}, \mathrm{CDCl}_{3}\right) \delta 7.40-7.19(\mathrm{~m}, 5 \mathrm{H})$, $5.27(\mathrm{~d}, \mathrm{~J}=1.7 \mathrm{~Hz}, 1 \mathrm{H}), 5.08(\mathrm{dd}, \mathrm{J}=2.0,1.0 \mathrm{~Hz}, 1 \mathrm{H}), 3.06-2.82(\mathrm{~m}, 1 \mathrm{H}), 2.74(\mathrm{~d}, \mathrm{~J}=13.8 \mathrm{~Hz}$, 1H), $2.23(\mathrm{~d}, \mathrm{~J}=13.8 \mathrm{~Hz}, 1 \mathrm{H}), 2.18-2.09(\mathrm{~m}, 1 \mathrm{H}), 1.75-1.41(\mathrm{~m}, 4 \mathrm{H}), 0.94(\mathrm{~m}, 6 \mathrm{H}), 0.91(\mathrm{~s}$, 3H). ${ }^{13} \mathrm{C}$ NMR (125 MHz, $\left.\mathrm{CDCl}_{3}\right) \delta 215.1,145.8,143.2,128.2,127.3,126.6,117.5,51.7,47.8$, 42.0, 36.3, 34.7, 34.4, 29.0, 28.3, 22.9. $[\alpha]^{22} \mathrm{D}=-25.8\left(\mathrm{c}=1.0, \mathrm{CHCl}_{3}\right)$. Enantiomeric excess was determined by HPLC with a Chiralpak AsH-H column (98:2, hexanes:isopropanol, $0.3 \mathrm{~mL} / \mathrm{min}$, $254 \mathrm{~nm})$; minor tr $=17.2 \mathrm{~min}$; major tr $=18.1 \mathrm{~min} ; 92 \%$ ee. HRMS $(\mathrm{ESI}) \mathrm{m} / \mathrm{z}:[\mathrm{M}+\mathrm{H}]+$ calcd for $\mathrm{C}_{18} \mathrm{H}_{24} \mathrm{O}$ 257.1900; Found 257.1908.
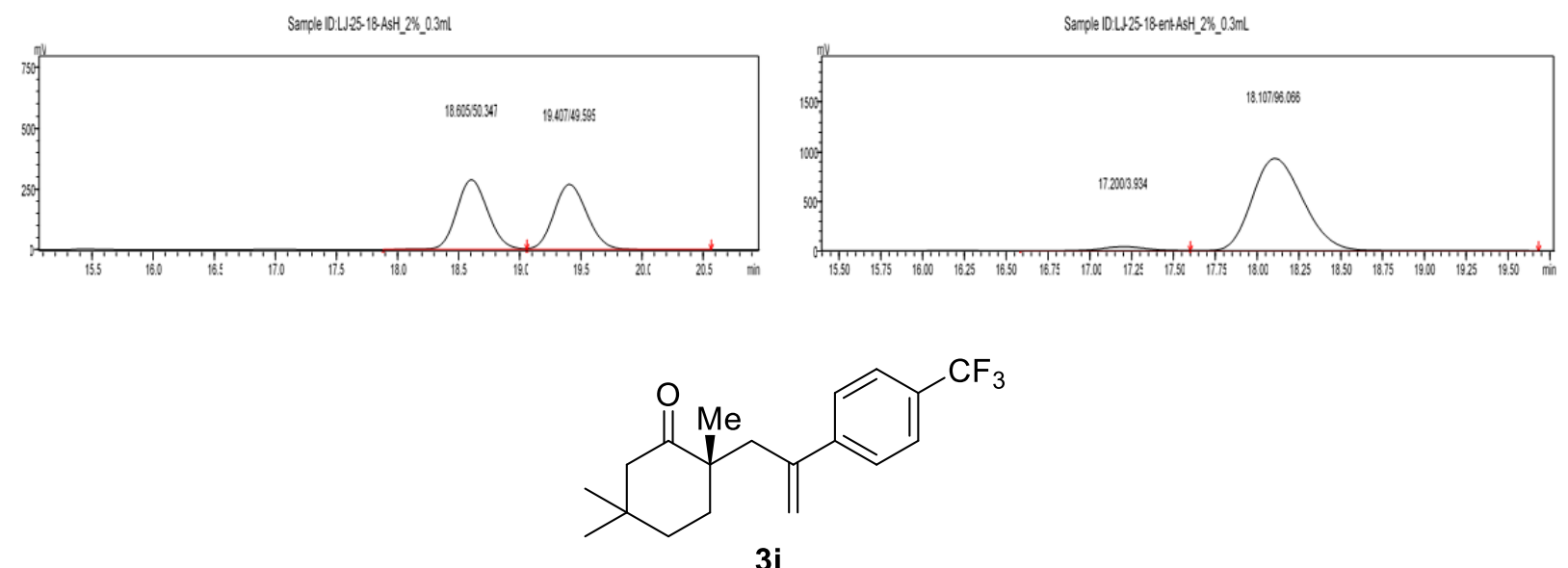

(S)-2,5,5-trimethyl-2-(2-(4-(trifluoromethyl)phenyl)allyl)cyclohexan-1-one

(3i).

Synthesized via common procedure II employing acid $\mathbf{1 h}$ and allyl carbonate $\mathbf{2} \mathbf{c}$ in $80 \%$ yield (51.7 $\mathrm{mg})$ as a colorless liquid. $\mathrm{R}_{\mathrm{f}}=0.73\left(20 \%\right.$ EtOAc/hexanes, UV-Vis). ${ }^{1} \mathrm{H} \mathrm{NMR}\left(500 \mathrm{MHz}, \mathrm{CDCl}_{3}\right)$ $\delta 7.57(\mathrm{~d}, J=8.1 \mathrm{~Hz}, 2 \mathrm{H}), 7.45(\mathrm{~d}, J=8.1 \mathrm{~Hz}, 2 \mathrm{H}), 5.33(\mathrm{~d}, J=1.4 \mathrm{~Hz}, 1 \mathrm{H}), 5.18(\mathrm{~d}, J=1.6 \mathrm{~Hz}$, 1H), $2.92(\mathrm{~d}, J=13.9 \mathrm{~Hz}, 1 \mathrm{H}), 2.75(\mathrm{~d}, J=13.9 \mathrm{~Hz}, 1 \mathrm{H}), 2.19(\mathrm{~s}, 2 \mathrm{H}), 1.73-1.43(\mathrm{~m}, 4 \mathrm{H}), 0.97$ $-0.87(\mathrm{~m}, 9 \mathrm{H}) .{ }^{13} \mathrm{C} \mathrm{NMR}\left(125 \mathrm{MHz}, \mathrm{CDCl}_{3}\right) \delta 214.8,146.9,144.8,129.3\left(\mathrm{q}, J_{F}=32.5 \mathrm{~Hz}\right), 126.8$, $125.2\left(\mathrm{q}, J_{F}=3.8 \mathrm{~Hz}\right), 123.0,119.4,51.7,47.8,41.9,36.3,34.8,34.3,28.63,28.59,23.0 .{ }^{19} \mathrm{~F}$ NMR 
$\left(300 \mathrm{MHz}, \mathrm{CDCl}_{3}\right) \delta-62.44 .[\alpha]^{22}{ }_{\mathrm{D}}=-17.9\left(\mathrm{c}=1.0, \mathrm{CHCl}_{3}\right)$. Enantiomeric excess was determined by HPLC with a Chiralpak AsH-H column (98:2, hexanes:isopropanol, $1 \mathrm{~mL} / \mathrm{min}, 254 \mathrm{~nm}$ ); minor $\operatorname{tr}=4.6 \mathrm{~min} ;$ major tr $=5.1 \mathrm{~min} ; 94 \%$ ee. HRMS (ESI) m/z: $[\mathrm{M}+\mathrm{H}]+$ calcd for $\mathrm{C}_{19} \mathrm{H}_{23} \mathrm{~F}_{3} \mathrm{O}$ 325.1744; Found 325.1757.
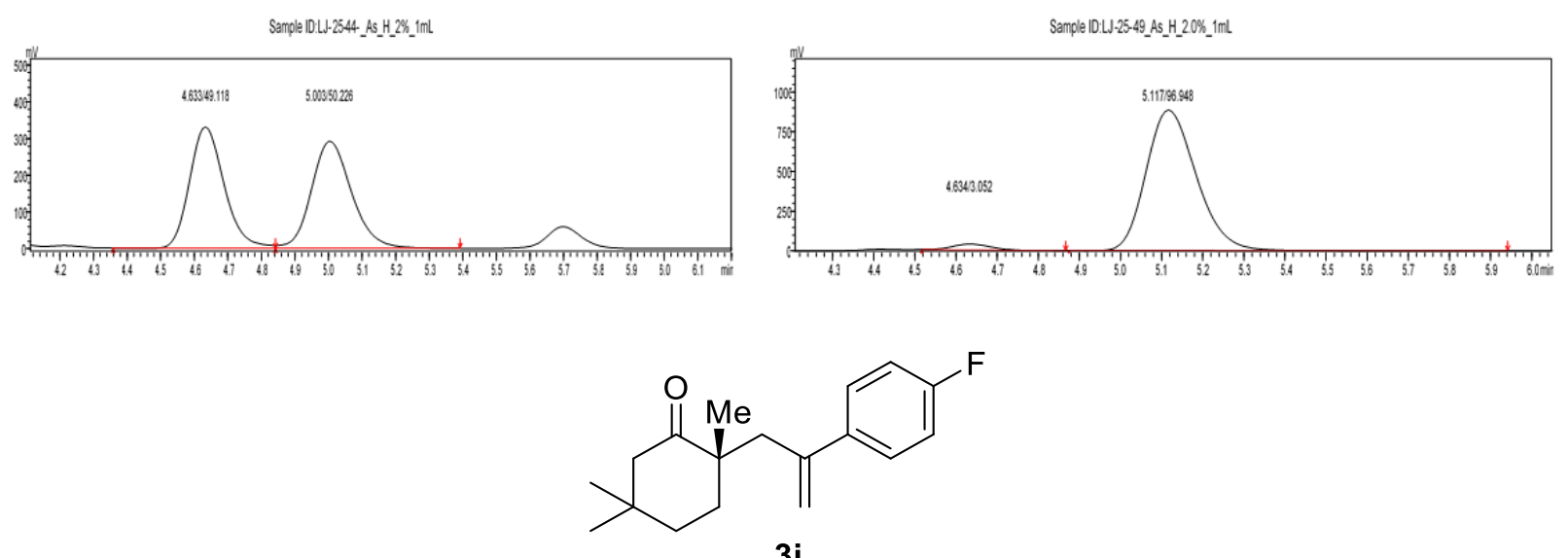

3j

(S)-2-(2-(4-fluorophenyl)allyl)-2,5,5-trimethylcyclohexan-1-one (3j). Synthesized via common procedure II employing acid $\mathbf{1 h}$ and allyl carbonate $\mathbf{2 b}$ in $83 \%$ yield $(45.6 \mathrm{mg}$ ) as a colorless liquid. $\mathrm{R}_{\mathrm{f}}=0.73$ (20\% EtOAc/hexanes, UV-Vis). ${ }^{1} \mathrm{H}$ NMR $\left(500 \mathrm{MHz}, \mathrm{CDCl}_{3}\right) \delta 7.44-$ $7.20(\mathrm{~m}, 2 \mathrm{H}), 7.10-6.91(\mathrm{~m}, 2 \mathrm{H}), 5.22(\mathrm{~d}, J=1.6 \mathrm{~Hz}, 1 \mathrm{H}), 5.06(\mathrm{~d}, J=1.6 \mathrm{~Hz}, 1 \mathrm{H}), 2.97-2.78$ (m, 1H), $2.70(\mathrm{~d}, J=13.8 \mathrm{~Hz}, 1 \mathrm{H}), 2.31-1.99(\mathrm{~m}, 2 \mathrm{H}), 1.77-1.44(\mathrm{~m}, 4 \mathrm{H}), 0.94(\mathrm{~m}, 6 \mathrm{H}), 0.91$ $(\mathrm{s}, 3 \mathrm{H}) \cdot{ }^{13} \mathrm{C} \mathrm{NMR}\left(125 \mathrm{MHz}, \mathrm{CDCl}_{3}\right) \delta 215.0,162.3\left(\mathrm{~d}, J_{\mathrm{F}}=240 \mathrm{~Hz}\right), 144.8,139.3\left(\mathrm{~d}, J_{\mathrm{F}}=2.5 \mathrm{~Hz}\right)$, $128.1\left(\mathrm{~d}, J_{\mathrm{F}}=7.5 \mathrm{~Hz}\right), 117.6,115.0\left(\mathrm{~d}, J_{\mathrm{F}}=21.0 \mathrm{~Hz}\right), 51.7,47.8,42.3,36.3,34.8,34.4,29.0,28.4$, 23.0. ${ }^{19} \mathrm{~F} \mathrm{NMR}\left(300 \mathrm{MHz}, \mathrm{CDCl}_{3}\right) \delta$-115.4. $[\alpha]^{20} \mathrm{D}=-24.4\left(\mathrm{c}=1.0, \mathrm{CHCl}_{3}\right)$. Enantiomeric excess was determined by HPLC with a Chiralpak As-H column (98:2, hexanes:isopropanol, $0.2 \mathrm{~mL} / \mathrm{min}$, $254 \mathrm{~nm}$ ); minor tr $=27.2 \mathrm{~min}$; major $\operatorname{tr}=29.3 \mathrm{~min} ; 92 \%$ ee. HRMS $(\mathrm{ESI}) \mathrm{m} / \mathrm{z}:[\mathrm{M}+\mathrm{H}]+$ calcd for $\mathrm{C}_{18} \mathrm{H}_{23} \mathrm{OF} 275.1806$; Found 275.1811. 

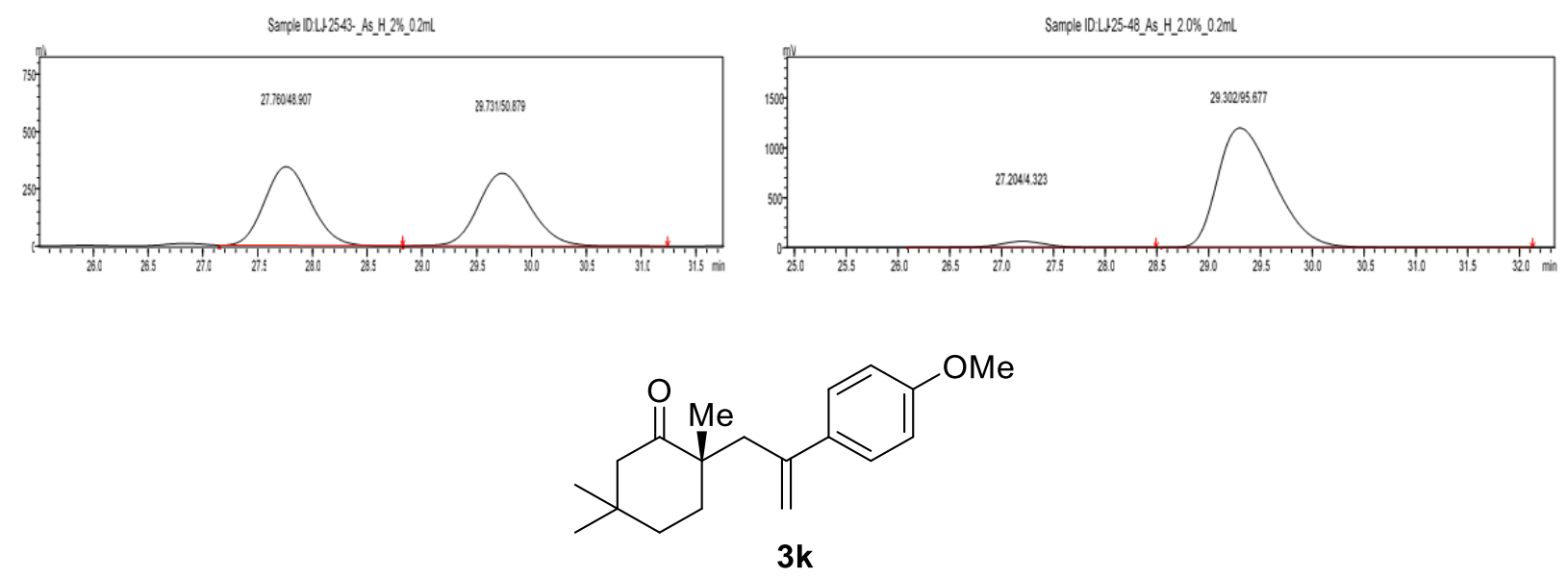

(S)-2-(2-(4-methoxyphenyl)allyl)-2,5,5-trimethylcyclohexan-1-one (3k). Synthesized via common procedure II employing acid $\mathbf{1 h}$ and allyl carbonate $\mathbf{2 d}$ in $81 \%$ yield (46.3 $\mathrm{mg}$ ) as a colorless liquid. $\mathrm{R}_{\mathrm{f}}=0.65\left(20 \%\right.$ EtOAc/hexanes, UV-Vis). ${ }^{1} \mathrm{H} \mathrm{NMR}\left(500 \mathrm{MHz}, \mathrm{CDCl}_{3}\right) \delta 7.37-$ $7.10(\mathrm{~m}, 2 \mathrm{H}), 6.98-6.70(\mathrm{~m}, 2 \mathrm{H}), 5.20(\mathrm{t}, J=2.1 \mathrm{~Hz}, 1 \mathrm{H}), 4.99(\mathrm{t}, J=2.2 \mathrm{~Hz}, 1 \mathrm{H}), 3.81(\mathrm{~s}, 3 \mathrm{H})$, $2.98-2.81(\mathrm{~m}, 1 \mathrm{H}), 2.70(\mathrm{dd}, J=13.9,2.6 \mathrm{~Hz}, 1 \mathrm{H}), 2.23(\mathrm{dd}, J=13.8,2.6 \mathrm{~Hz}, 1 \mathrm{H}), 2.15(\mathrm{dt}, J=$ 13.9, $1.7 \mathrm{~Hz}, 1 \mathrm{H}), 1.72-1.37(\mathrm{~m}, 6 \mathrm{H}), 0.94(\mathrm{~m}, 4 \mathrm{H}), 0.90(\mathrm{~s}, 3 \mathrm{H}) .{ }^{13} \mathrm{C} \mathrm{NMR}\left(125 \mathrm{MHz}, \mathrm{CDCl}_{3}\right)$ $\delta 215.2,158.9,145.1,135.7,127.6,116.2,113.5,55.2,51.7,47.8,42.0,36.3,34.7,34.4,29.1$, 28.3, 23.0. $[\alpha]^{22} \mathrm{D}=-24.08\left(\mathrm{c}=1.0, \mathrm{CHCl}_{3}\right)$. Enantiomeric excess was determined by HPLC with a Chiralpak As-H column (98:2, hexanes:isopropanol, $1 \mathrm{~mL} / \mathrm{min}, 254 \mathrm{~nm})$; minor tr = $7.0 \mathrm{~min}$; major $\operatorname{tr}=7.7 \mathrm{~min} ; 92 \%$ ee. HRMS $(\mathrm{ESI}) \mathrm{m} / \mathrm{z}$ : $[\mathrm{M}+\mathrm{H}]+$ calcd for $\mathrm{C}_{19} \mathrm{H}_{26} \mathrm{O}_{2}$ 287.2006; Found 287.2016.
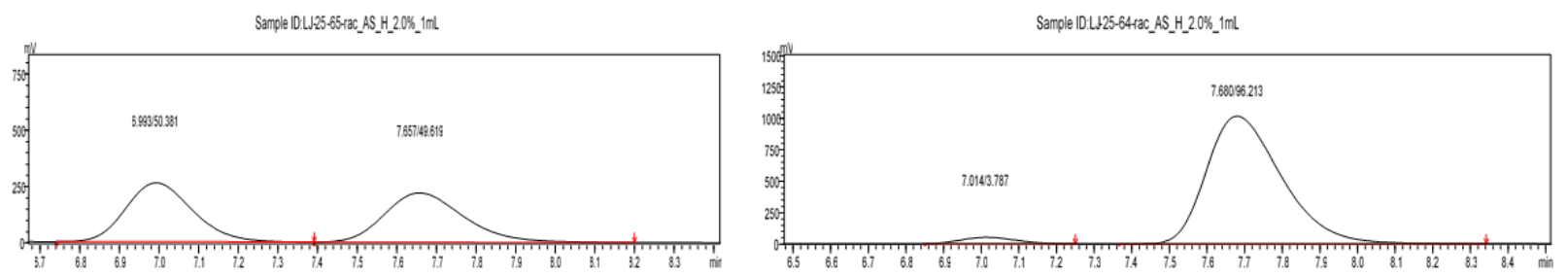


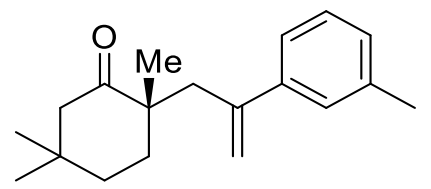

3I

(S)-2,5,5-trimethyl-2-(2-(m-tolyl)allyl)cyclohexan-1-one (3l). Synthesized via common procedure II employing acid $\mathbf{1 h}$ and allyl carbonate $\mathbf{2 e}$ in $83 \%$ yield (48.0 $\mathrm{mg}$ ) as a colorless liquid. $\mathrm{R}_{\mathrm{f}}=0.75$ (20\% EtOAc/hexanes, UV-Vis). ${ }^{1} \mathrm{H} \mathrm{NMR}(600 \mathrm{MHz}, \mathrm{CDCl} 3) \delta 7.26-7.07$ (m, 4H), $5.26(\mathrm{~d}, J=1.8 \mathrm{~Hz}, 1 \mathrm{H}), 5.06(\mathrm{~d}, J=1.6 \mathrm{~Hz}, 1 \mathrm{H}), 2.90(\mathrm{dd}, J=13.8,0.9 \mathrm{~Hz}, 1 \mathrm{H}), 2.73(\mathrm{~d}, J=13.8$ $\mathrm{Hz}, 1 \mathrm{H}), 2.37(\mathrm{~s}, 3 \mathrm{H}), 2.28-2.21(\mathrm{~m}, 1 \mathrm{H}), 2.21-2.12(\mathrm{~m}, 1 \mathrm{H}), 1.70-1.56(\mathrm{~m}, 2 \mathrm{H}), 1.55-1.44$ $(\mathrm{m}, 2 \mathrm{H}), 0.96(\mathrm{~s}, 3 \mathrm{H}), 0.94(\mathrm{~s}, 3 \mathrm{H}), 0.92(\mathrm{~s}, 3 \mathrm{H}) .{ }^{13} \mathrm{C}$ NMR (150 MHz, $\left.\mathrm{CDCl}_{3}\right) \delta$ 215.3, 145.9, 143.2, 137.7, 128.1, 128.0, 127.3, 123.6, 117.2, 51.7, 47.8, 42.0, 36.3, 34.7, 34.4, 29.1, 28.3, 22.9, 21.5. $[\alpha]^{20} \mathrm{D}=-22.6\left(\mathrm{c}=1.0, \mathrm{CHCl}_{3}\right)$. Enantiomeric excess was determined by HPLC with a Chiralpak As-H column (99.5:0.5, hexanes:isopropanol, $1 \mathrm{~mL} / \mathrm{min}, 254 \mathrm{~nm}$ ); minor tr = $10.2 \mathrm{~min}$; major $\operatorname{tr}=11.3 \mathrm{~min} ; 92 \%$ ee. HRMS (ESI) m/z: $[\mathrm{M}+\mathrm{H}]+$ calcd for $\mathrm{C}_{19} \mathrm{H}_{26} \mathrm{O}$ 271.2056; Found 271.2067 .
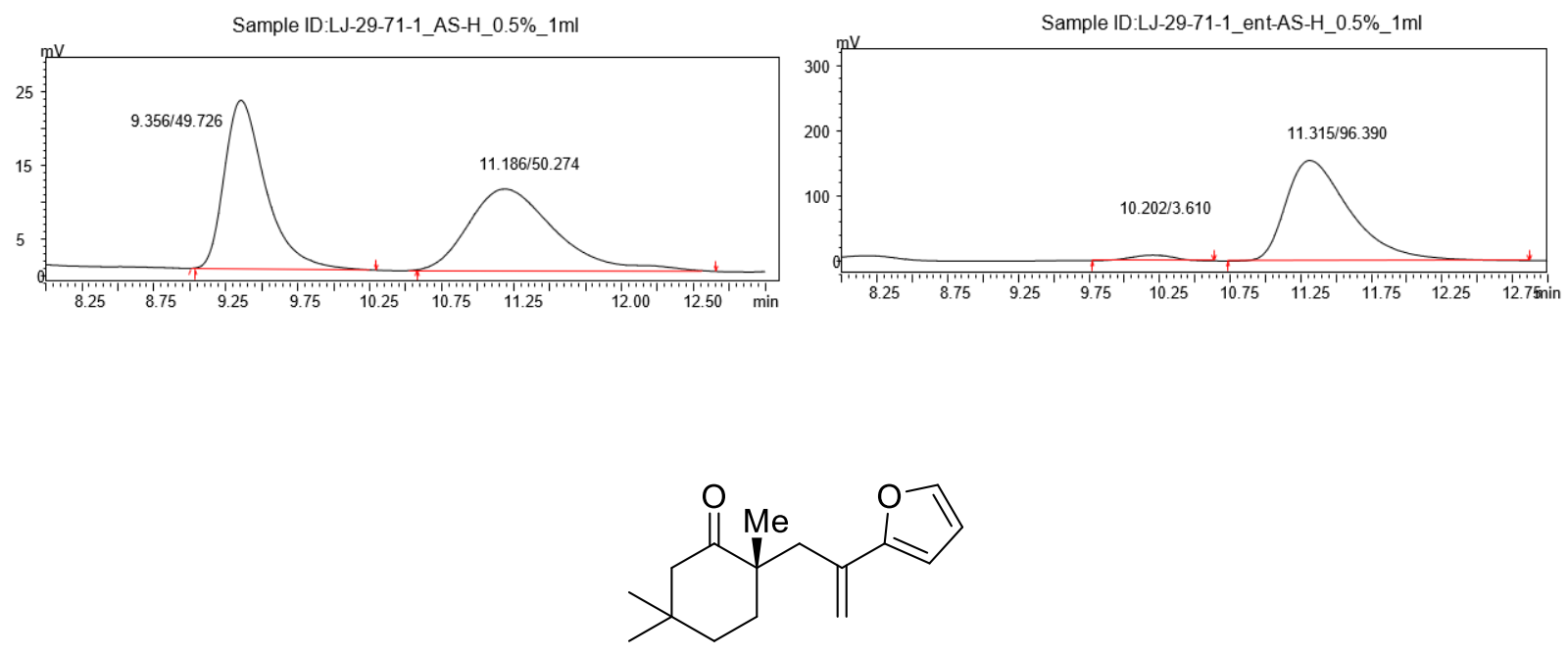

$3 \mathrm{~m}$

(S)-2-(2-(furan-2-yl)allyl)-2,5,5-trimethylcyclohexan-1-one $\quad(3 \mathrm{~m}) . \quad$ Synthesized via common procedure II employing acid $\mathbf{1 h}$ and allyl carbonate $\mathbf{2 f}$ in $61 \%$ yield $(30.0 \mathrm{mg}$ ) as a 
colorless liquid. $\mathrm{R}_{\mathrm{f}}=0.51\left(20 \%\right.$ EtOAc/hexanes, UV-Vis). ${ }^{1} \mathrm{H}$ NMR $\left(600 \mathrm{MHz}, \mathrm{CDCl}_{3}\right) \delta 7.34(\mathrm{~d}$, $J=1.7 \mathrm{~Hz}, 1 \mathrm{H}), 6.45-6.20(\mathrm{~m}, 2 \mathrm{H}), 5.63(\mathrm{~d}, J=1.3 \mathrm{~Hz}, 1 \mathrm{H}), 4.96(\mathrm{~d}, J=1.3 \mathrm{~Hz}, 1 \mathrm{H}), 2.75(\mathrm{dd}$, $J=14.1,0.9 \mathrm{~Hz}, 1 \mathrm{H}), 2.63(\mathrm{~d}, J=14.1 \mathrm{~Hz}, 1 \mathrm{H}), 2.38-2.22(\mathrm{~m}, 2 \mathrm{H}), 1.84-1.73(\mathrm{~m}, 1 \mathrm{H}), 1.69-$ $1.54(\mathrm{~m}, 3 \mathrm{H}), 1.06$ (s, 3H), 0.97 (s, 3H), 0.96 (s, 3H). ${ }^{13} \mathrm{C} \mathrm{NMR}\left(150 \mathrm{MHz}, \mathrm{CDCl}_{3}\right) \delta$ 215.2, 155.5, $141.7,134.0,113.8,111.2,106.5,51.7,47.6,39.3,36.4,34.5,34.4,28.7,28.7,22.8 .[\alpha]^{20} \mathrm{D}=-10.3$ $\left(\mathrm{c}=1.0, \mathrm{CHCl}_{3}\right)$. Enantiomeric excess was determined by HPLC with a Chiralcel OD-H column (99.7:0.3, hexanes:isopropanol, $1 \mathrm{~mL} / \mathrm{min}, 254 \mathrm{~nm})$; $\operatorname{minor} \operatorname{tr}=18.2 \mathrm{~min} ;$ major $\operatorname{tr}=19.7 \mathrm{~min}$; 94\% ee. HRMS (ESI) m/z: [M+H]+ calcd for $\mathrm{C}_{16} \mathrm{H}_{22} \mathrm{O}_{2}$ 247.1693; Found 247.1701.
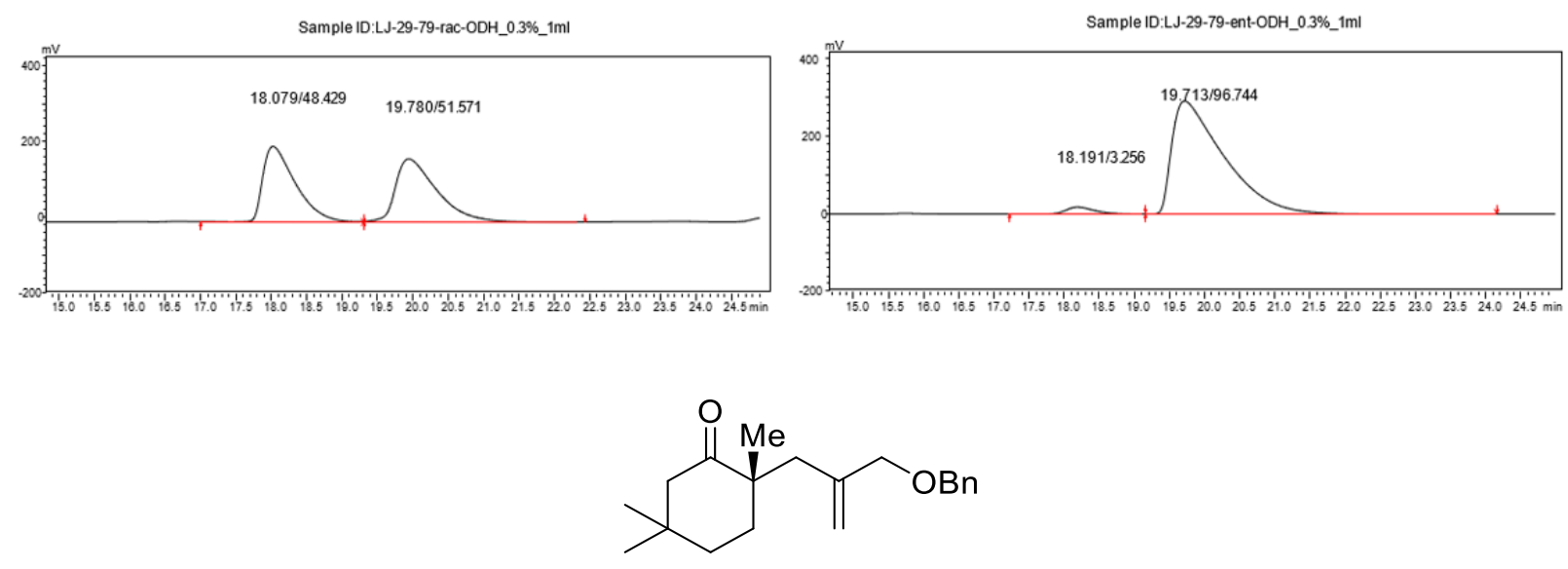

$3 n$

(S)-2-(2-((benzyloxy)methyl)allyl)-2,5,5-trimethylcyclohexan-1-one. was synthesized via common procedure II employing acid $\mathbf{1 h}$ and allyl carbonate $\mathbf{2} \mathbf{i}$ in $90 \%$ yield $(54.1 \mathrm{mg}$ ) as a colorless liquid. $\mathrm{R}_{\mathrm{f}}=0.60$ (20\% EtOAc/hexanes, UV-Vis). ${ }^{1} \mathrm{H} \mathrm{NMR}\left(500 \mathrm{MHz}, \mathrm{CDCl}_{3}\right) \delta 7.43-$ $7.27(\mathrm{~m}, 5 \mathrm{H}), 5.19(\mathrm{q}, J=1.5 \mathrm{~Hz}, 1 \mathrm{H}), 4.94(\mathrm{~d}, J=1.8 \mathrm{~Hz}, 1 \mathrm{H}), 4.54-4.36(\mathrm{~m}, 2 \mathrm{H}), 3.94-3.73$ (m, 2H), $2.69(\mathrm{~d}, J=14.1 \mathrm{~Hz}, 1 \mathrm{H}), 2.47(\mathrm{~d}, J=13.6 \mathrm{~Hz}, 1 \mathrm{H}), 2.21(\mathrm{~d}, J=14.2 \mathrm{~Hz}, 1 \mathrm{H}), 2.13(\mathrm{dt}$, $J=13.6,1.7 \mathrm{~Hz}, 1 \mathrm{H}), 1.90-1.63(\mathrm{~m}, 3 \mathrm{H}), 1.54-1.39(\mathrm{~m}, 1 \mathrm{H}), 1.07(\mathrm{~d}, J=1.4 \mathrm{~Hz}, 3 \mathrm{H}), 0.99(\mathrm{~d}$, $J=1.3 \mathrm{~Hz}, 3 \mathrm{H}), 0.92(\mathrm{~d}, J=1.3 \mathrm{~Hz}, 3 \mathrm{H}) .{ }^{13} \mathrm{C} \mathrm{NMR}\left(125 \mathrm{MHz}, \mathrm{CDCl}_{3}\right) \delta 215.7,142.5,138.2$ $128.4,127.7,127.6,116.4,73.7,72.0,51.7,47.5,40.5,36.8,36.3,34.4,30.0,27.3,22.7 .[\alpha]^{20}{ }_{D}=$ 
$-17.1\left(\mathrm{c}=1.0, \mathrm{CHCl}_{3}\right)$. Enantiomeric excess was determined by HPLC with a Chiralcel OD-H column (99:1, hexanes:isopropanol, $1 \mathrm{~mL} / \mathrm{min}, 254 \mathrm{~nm})$; $\operatorname{minor} \operatorname{tr}=7.9 \mathrm{~min}$; major $\operatorname{tr}=8.5 \mathrm{~min}$; 92\% ee. HRMS (ESI) m/z: [M+H]+ calcd for $\mathrm{C}_{20} \mathrm{H}_{28} \mathrm{O}_{2}$ 301.2162; Found 301.2166.
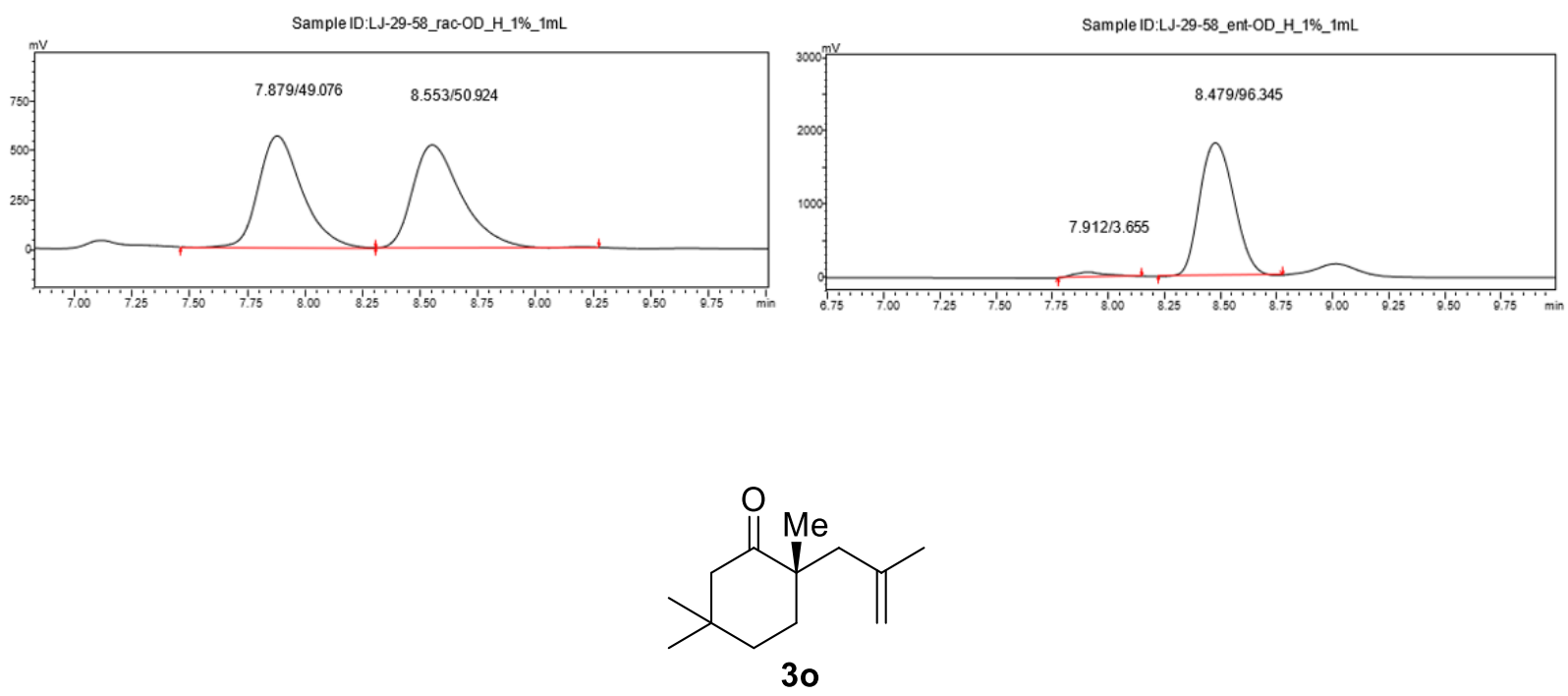

(S)-2,5,5-trimethyl-2-(2-methylallyl)cyclohexan-1-one one was synthesized via common procedure II employing acid $\mathbf{1 h}$ and allyl carbonate $\mathbf{2 h}$ in $83 \%$ yield $(32.0 \mathrm{mg})$ as a colorless liquid. $\mathrm{R}_{\mathrm{f}}=0.70\left(20 \%\right.$ EtOAc/hexanes, UV-Vis). ${ }^{1} \mathrm{H}$ NMR $\left(300 \mathrm{MHz}, \mathrm{CDCl}_{3}\right) \delta 4.82(\mathrm{p}$ $J=1.6 \mathrm{~Hz}, 1 \mathrm{H}), 4.72-4.62(\mathrm{~m}, 1 \mathrm{H}), 2.51-2.31(\mathrm{~m}, 2 \mathrm{H}), 2.20-2.04(\mathrm{~m}, 2 \mathrm{H}), 1.78-1.65(\mathrm{~m}$, 6H), $1.47(\mathrm{dt}, J=9.1,3.1 \mathrm{~Hz}, 1 \mathrm{H}), 1.05(\mathrm{~s}, 3 \mathrm{H}), 0.99(\mathrm{~s}, 3 \mathrm{H}), 0.90(\mathrm{~s}, 3 \mathrm{H}) .{ }^{13} \mathrm{C}$ NMR $(125 \mathrm{MHz}$, $\left.\mathrm{CDCl}_{3}\right) \delta 215.5,142.1,114.8,51.8,47.5,45.3,36.7,35.9,34.3,30.0,27.3,24.3,22.8 .[\alpha]^{22} \mathrm{D}=-$ $19.5(\mathrm{c}=1.0, \mathrm{DCM})$. Enantiomeric excess was determined based on the O-Bn oxime of the ketone 3o by HPLC with a Chiralcel OD-H column (99.7:0.3, hexanes:isopropanol, $1 \mathrm{~mL} / \mathrm{min}, 254 \mathrm{~nm}$ ); minor $\operatorname{tr}=15.1 \mathrm{~min} ;$ major $\operatorname{tr}=21.0 \mathrm{~min} ; 91 \%$ ee. HRMS (ESI) m/z: $[\mathrm{M}+\mathrm{H}]+$ calcd for $\mathrm{C}_{13} \mathrm{H}_{22} \mathrm{O}$ 195.1743; Found 195.1746. 

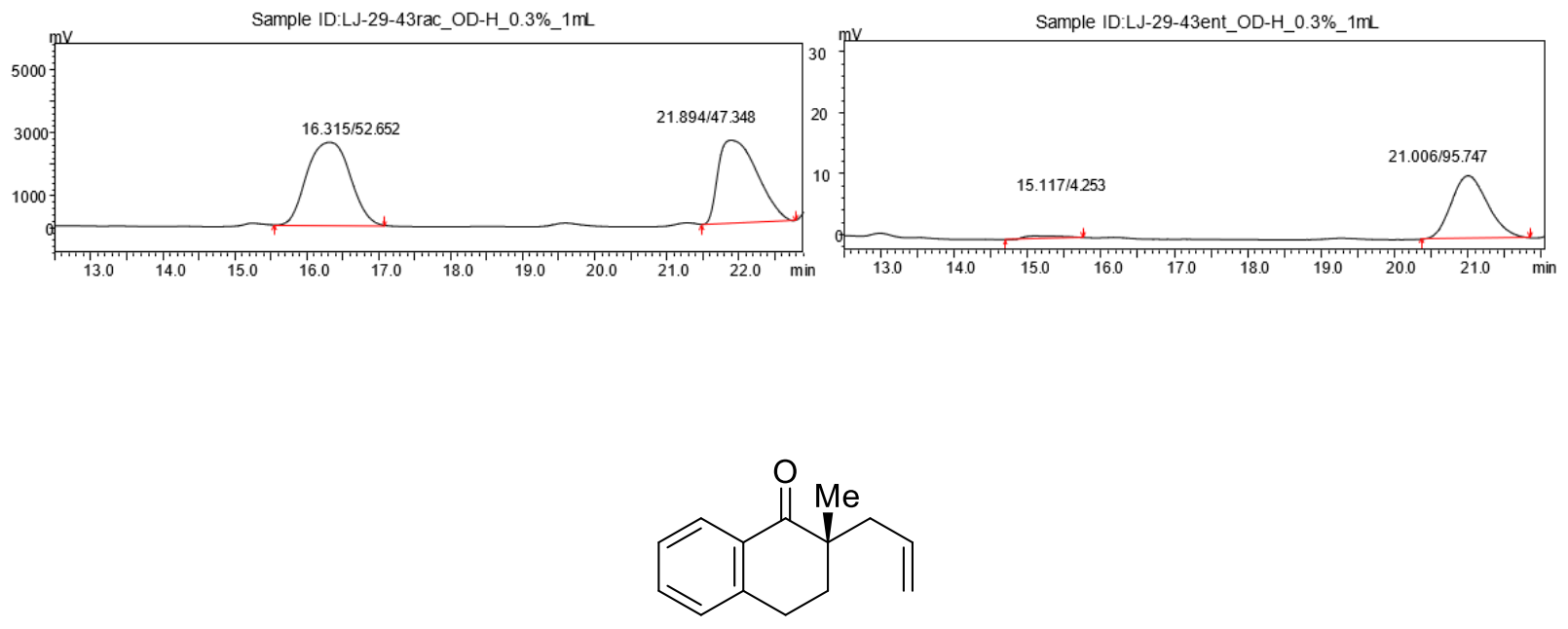

$3 p$

(S)-2-allyl-2-methyl-3,4-dihydronaphthalen-1(2H)-one (3p) was synthesized via common procedure II employing acid $\mathbf{1 p}$ and allyl carbonate $\mathbf{2 i}$ in $65 \%$ yield (26 $\mathrm{mg}$ ) as a colorless liquid. $\mathrm{R}_{\mathrm{f}}=0.75(20 \%$ EtOAc/hexanes, UV-Vis $) .[\alpha]^{22} \mathrm{D}=-11.8\left(\mathrm{c}=1.0, \mathrm{CHCl}_{3}\right)$. Enantiomeric excess was determined by HPLC with a Chiralcel OB-H column (97:3, hexanes:isopropanol, 1 $\mathrm{mL} / \mathrm{min}, 254 \mathrm{~nm}$ ); minor $\mathrm{tr}=4.6 \mathrm{~min}$; major $\mathrm{tr}=5.1 \mathrm{~min} ; 89 \%$ ee. The ${ }^{1} \mathrm{H} \mathrm{NMR}$ and ${ }^{13} \mathrm{C} \mathrm{NMR}$ matched with reported data. ${ }^{6}$
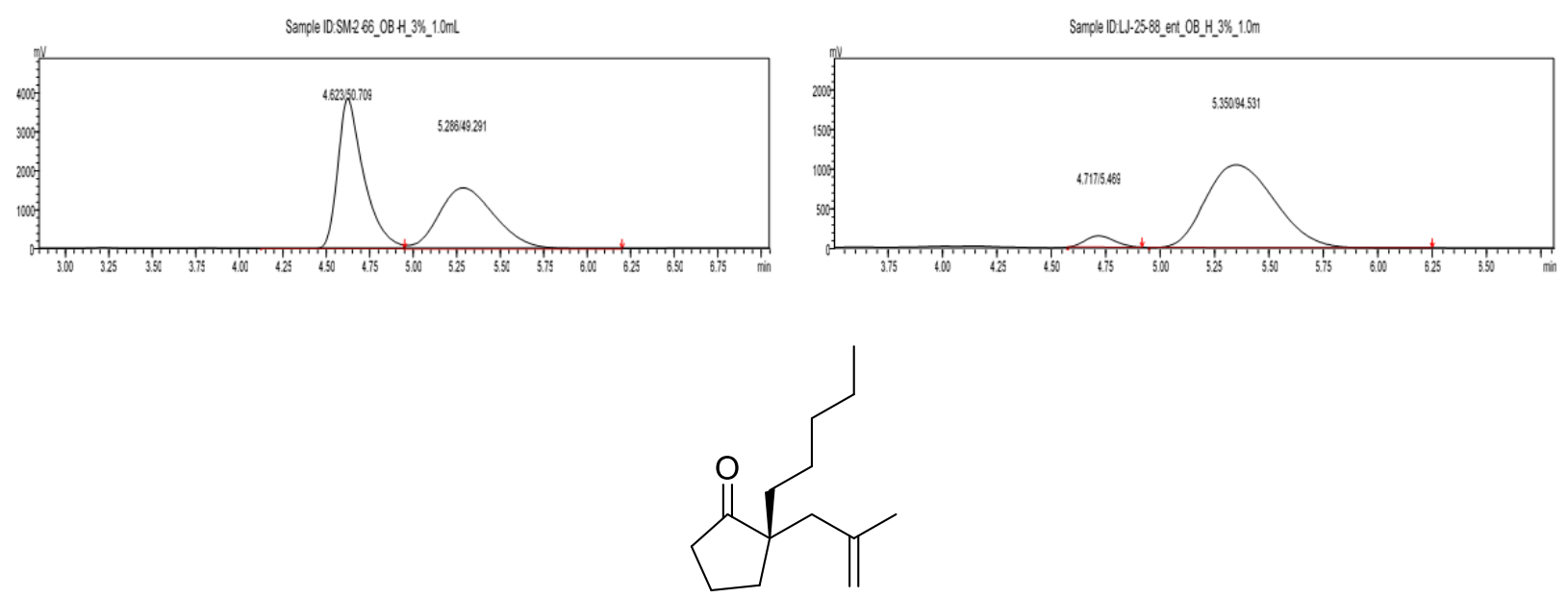

$3 \mathbf{q}$

(S)-2-(2-methylallyl)-2-pentylcyclopentan-1-one. Condition $\mathrm{A}: \mathrm{Pd}_{2} \mathrm{dba}_{3} \cdot \mathrm{CHCl}_{3}(5.1 \mathrm{mg}$, $0.005 \mathrm{mmol}$ ), (S)-tBu-Phox (4.8 $\mathrm{mg}, 0.0125 \mathrm{mmol}$ ) and NaHMDS (37 mg, $0.2 \mathrm{mmol}$ ) were weighed out in an oven-dried round bottom flask in a glove box. The flask was sealed with a 
septum, taken out and connected with an argon balloon via a needle. THF (2.0 mL) was added and the resulting solution was allowed to stir at r.t. for $30 \mathrm{~min}$. Carboxylic acid 1q $(39.0 \mathrm{mg}, 0.2 \mathrm{mmol}$ in $2 \mathrm{~mL}$ THF) and allyl carbonate $\mathbf{2 h}(26 \mathrm{mg}, 0.2 \mathrm{mmol}$ in $2 \mathrm{~mL}$ THF) were added to the prestirred catalyst solution via syringe. After stirring under argon at $40{ }^{\circ} \mathrm{C}$. for $12 \mathrm{~h}$, the reaction mixture was adsorbed onto silica and purified by column chromatography to give desired product in $67 \%$ yield $(27.8 \mathrm{mg})$ as a colorless oil. Enantiomeric excess was determined based on the O-Bn oxime of the ketone 3q by HPLC with a Chiralpak ODH-H column (hexanes, $0.3 \mathrm{~mL} / \mathrm{min}, 254$ $\mathrm{nm}$ ); $\operatorname{minor} \operatorname{tr}=19.7 \mathrm{~min}$; major $\operatorname{tr}=22.2 \mathrm{~min} ; 83 \%$ ee.
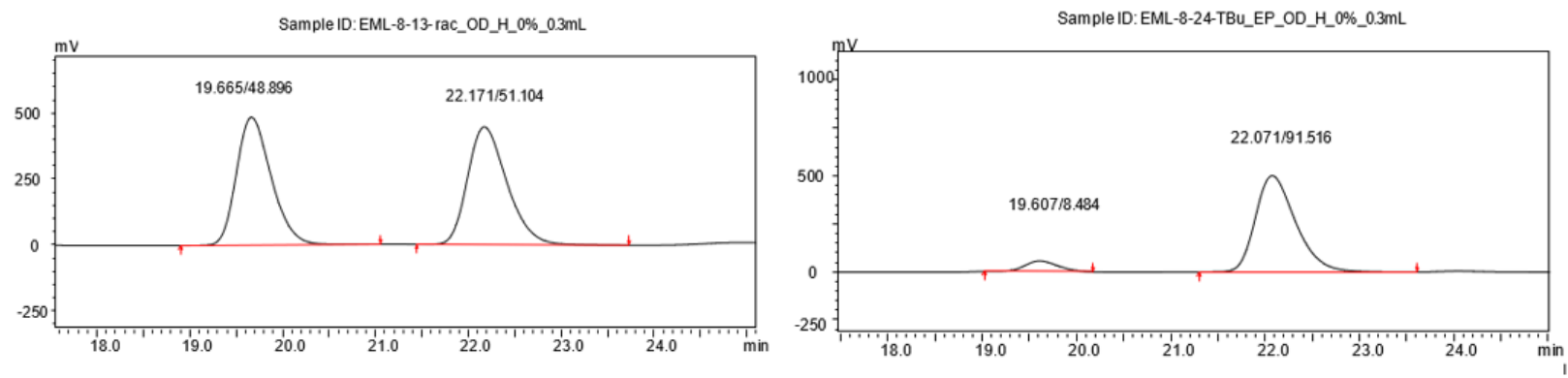

Condition B: $\mathrm{Pd}_{2} \mathrm{dba}_{3} \cdot \mathrm{CHCl}_{3}(5.1 \mathrm{mg}, 0.005 \mathrm{mmol}),(S)-t \mathrm{Bu}-\left(\mathrm{CF}_{3}\right)_{3} \mathrm{Phox}(7.8 \mathrm{mg}, 0.0125$ $\mathrm{mmol}$ ) and NaHMDS (37 mg, $0.2 \mathrm{mmol}$ ) were weighed out in an oven-dried round bottom flask in a glove box. The flask was sealed with a septum, taken out and connected with an argon balloon via a needle. THF $(2.0 \mathrm{~mL})$ was added and the resulting solution was allowed to stir at r.t. for 30 min. Carboxylic acid 1q (39 mg, $0.2 \mathrm{mmol}$ in $2 \mathrm{~mL} \mathrm{THF})$ and allyl carbonate $\mathbf{2 h}(26 \mathrm{mg}, 0.2 \mathrm{mmol}$ in $2 \mathrm{~mL}$ THF) were added to the pre-stirred catalyst solution via syringe. After stirring under argon at $40{ }^{\circ} \mathrm{C}$. for $12 \mathrm{~h}$, the reaction mixture was adsorbed onto silica and purified by column chromatography to give desired product in $61 \%$ yield $(25.3 \mathrm{mg})$ as a colorless oil. $\mathrm{R}_{\mathrm{f}}=0.70(20 \%$ EtOAc/hexanes, UV-Vis). ${ }^{1} \mathrm{H}$ NMR $\left(500 \mathrm{MHz}, \mathrm{CDCl}_{3}\right) \delta 7.43-7.27(\mathrm{~m}, 5 \mathrm{H}), 5.30(\mathrm{~d}, \mathrm{~J}=1.7 \mathrm{~Hz}$, 1H), $5.17-5.01(\mathrm{~m}, 1 \mathrm{H}), 2.79-2.73(\mathrm{~m}, 1 \mathrm{H}), 2.65(\mathrm{~d}, J=13.8 \mathrm{~Hz}, 1 \mathrm{H}), 2.30-2.19(\mathrm{~m}, 1 \mathrm{H}), 2.08$ $-1.93(\mathrm{~m}, 1 \mathrm{H}), 1.90-1.65(\mathrm{~m}, 3 \mathrm{H}), 1.54-1.41(\mathrm{~m}, 1 \mathrm{H}), 0.94(\mathrm{~s}, 3 \mathrm{H}) .{ }^{13} \mathrm{C} \mathrm{NMR}(125 \mathrm{MHz}$, $\left.\mathrm{CDCl}_{3}\right) \delta 223.1,146.0,142.3,128.2,127.4,126.7,117.1,48.9,41.7,37.4,35.2,22.5,18.6 .[\alpha]^{20}$ 
$\mathrm{D}=-19.5(\mathrm{c}=1.0, \mathrm{DCM})$. Enantiomeric excess was determined based on the O-Bn oxime of the ketone 3q HPLC with a Chiralpak ODH-H column (hexanes, $0.3 \mathrm{~mL} / \mathrm{min}, 254 \mathrm{~nm}$ ); minor $\mathrm{tr}=$ $19.7 \mathrm{~min}$; major $\operatorname{tr}=22.2 \mathrm{~min}$; 85\% ee. $\mathrm{HRMS}(\mathrm{ESI}) \mathrm{m} / \mathrm{z}:[\mathrm{M}+\mathrm{H}]+$ calcd for $\mathrm{C}_{15} \mathrm{H}_{18} \mathrm{O} 215.1430$; Found 215.1420.
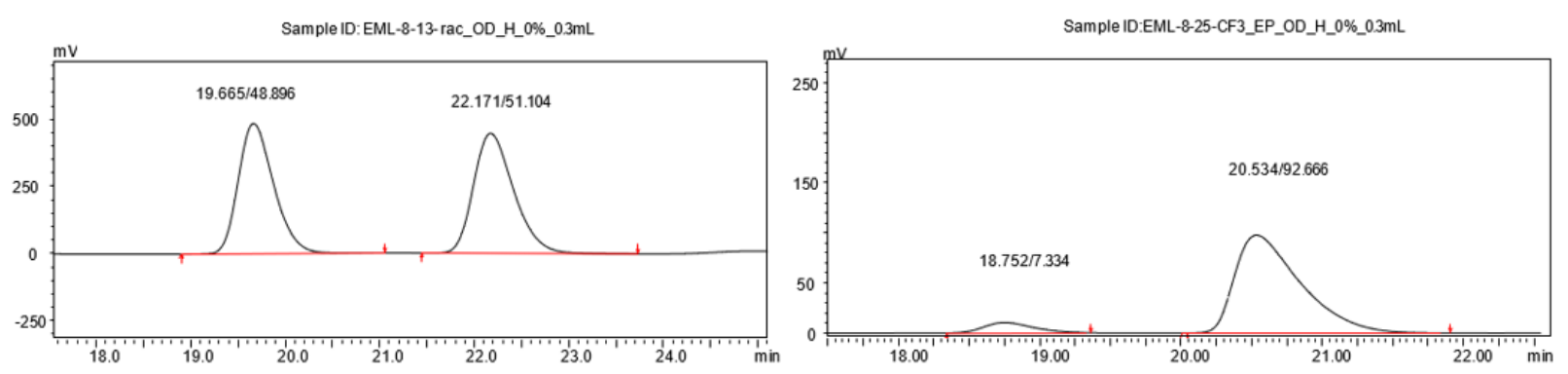

\section{Synthesis of Adalinine}

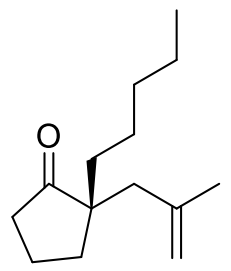

$3 q$

(S)-2-(2-methylallyl)-2-pentylcyclopentan-1-one (3q). $\mathrm{Pd}_{2} \mathrm{dba}_{3} \cdot \mathrm{CHCl}_{3}(64 \mathrm{mg}, 0.0625$ mmol), (S)-tBu-( $\left(\mathrm{CF}_{3}\right)_{3}$ Phox (98 mg, $\left.0.156 \mathrm{mmol}\right)$ and NaHMDS (462 mg, $2.5 \mathrm{mmol}$ ) were weighed out in an oven-dried round bottom flask in a glove box. The flask was sealed with a septum, taken out and connected with an argon balloon via a needle. THF $(25.0 \mathrm{~mL})$ was added and the resulting solution was allowed to stir at r.t. for $30 \mathrm{~min}$. Carboxylic acid $\mathbf{1 f}$ (489 mg, 2.5 mmol in $25 \mathrm{~mL}$ THF) and allyl carbonate $2 \mathbf{h}$ ( $325 \mathrm{mg}, 2.5 \mathrm{mmol}$ in $25 \mathrm{~mL}$ THF) were added to the pre-stirred catalyst solution via syringe. After stirring under argon at $40{ }^{\circ} \mathrm{C}$. for $12 \mathrm{~h}$, the reaction mixture was adsorbed onto silica and purified by column chromatography to give desired product in 59\% yield $(307 \mathrm{mg}$ ) as a colorless oil. Enantiomeric excess was determined based on the O-Bn oxime of the ketone 3q HPLC with a Chiralpak ODH-H column (hexanes, $0.3 \mathrm{~mL} / \mathrm{min}$, $254 \mathrm{~nm}$ ); minor tr $=19.7 \mathrm{~min}$; major $\operatorname{tr}=22.2 \mathrm{~min} ; 86 \%$ ee. 

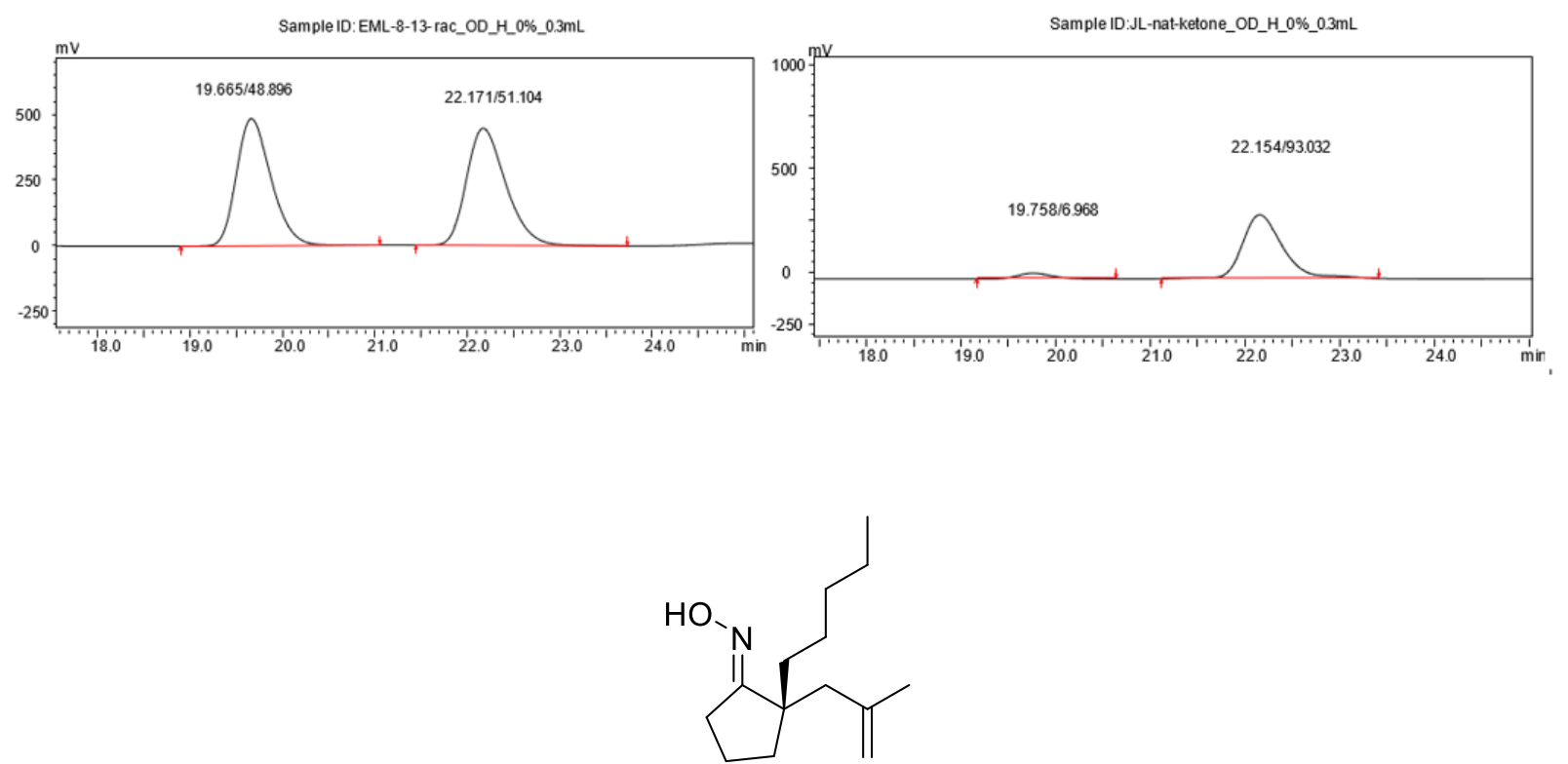

$3 q 1$

(E)-2-(2-methylallyl)-2-pentylcyclopentan-1-one oxime (3q1). To a solution of ketone 3q (150 mg, $0.72 \mathrm{mmol})$ in ethanol (7.2 mL, 200 proof) was added sodium acetate (236.3 mg, 2.88 $\mathrm{mmol}$ ) and hydroxylamine hydrochloride $(150.1 \mathrm{mg}, 2.16 \mathrm{mmol})$, respectively. After the reaction was refluxed at $85^{\circ} \mathrm{C}$ for $16 \mathrm{~h}$, the reaction solvent was stripped under reduced pressure. The crude mixture was diluted with ethyl acetate, dried over anhydrous sodium sulfate, filtered, and evaporated under reduced pressure. The crude material was purified via flash column chromatography on silica gel (1-3\% EtOAc/hexanes) to afford pure oxime 3q1 (136.6 mg, 0.61 $\mathrm{mmol})$ in $85 \%$ yield as a colorless oil. $\mathrm{R}_{\mathrm{f}}=0.39(10 \%$ EtOAc/hexanes, KMnO4 stain). [ $\alpha] 22 \mathrm{D}=$ $7.8\left(\mathrm{c}=1.00, \mathrm{CHCl}_{3}\right) .1 \mathrm{H} \mathrm{NMR}(600 \mathrm{MHz}, \mathrm{CDCl} 3): \delta 8.20(\mathrm{~s}, 1 \mathrm{H}), 4.83(\mathrm{~s}, 1 \mathrm{H}), 4.69(\mathrm{~s}, 1 \mathrm{H})$, $2.61-2.40(\mathrm{~m}, 2 \mathrm{H}), 2.26(\mathrm{~s}, 2 \mathrm{H}), 1.87-1.56(\mathrm{~m}, 7 \mathrm{H}), 1.55-1.38(\mathrm{~m}, 2 \mathrm{H}), 1.39-1.11(\mathrm{~m}, 6 \mathrm{H})$, $0.87(\mathrm{t}, J=7.2 \mathrm{~Hz}, 3 \mathrm{H}) .{ }^{13} \mathrm{C} \mathrm{NMR}\left(150 \mathrm{MHz}, \mathrm{CDCl}_{3}\right): \delta 223.3,142.7,114.9,51.9,43.4,38.3$, $36.5,32.7,32.5,24.4,23.9,22.6,18.8,14.1$. HRMS (ESI) calcd for $\mathrm{C}_{14} \mathrm{H}_{25} \mathrm{NO}(\mathrm{M}+\mathrm{H})^{+} 224.2009$, found 224.2020 .

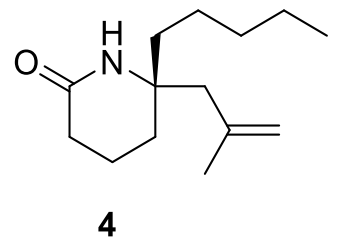

6-(2-methylallyl)-6-pentylpiperidin-2-one (4). To a solution of (E)-2-(2-methylallyl)-2pentylcyclopentan-1-one oxime $(99.1 \mathrm{mg}, 0.444 \mathrm{mmol})$ in pyridine $(7.7 \mathrm{~mL})$ was added DMAP 
(1.1 mg, $2 \mathrm{~mol} \%)$ and $p$-toluenesulfonyl chloride $(211.5 \mathrm{mg}, 1.11 \mathrm{mmol})$. The mixture was stirred at room temperature for $0.5 \mathrm{~h}$, then heated to $80^{\circ} \mathrm{C}$ for $3.5 \mathrm{~h}$. The solvent was then stripped under reduced pressure and the crude material was diluted with dichloromethane and washed with $1 \mathrm{M}$ $\mathrm{HCl}$ followed by a wash with saturated aqueous $\mathrm{NaHCO}_{3}$. The organic layer was then dried over sodium sulfate, filtered, and stripped of solvent under reduced pressure. The crude material was purified via flash column chromatography on silica gel $(0.5-1 \% \mathrm{MeOH} / \mathrm{DCM})$ to afford pure lactam $4(68.6 \mathrm{mg}, 0.307 \mathrm{mmol})$ in $69 \%$ yield as a clear amber oil. $\mathrm{R}_{\mathrm{f}}=0.52(10 \% \mathrm{MeOH} / \mathrm{DCM}$, $\mathrm{KMnO}_{4}$ stain). $[\alpha]^{22}{ }_{\mathrm{D}}=-21.1\left(\mathrm{c}=1.00, \mathrm{CHCl}_{3}\right) .{ }^{1} \mathrm{H} \mathrm{NMR}\left(400 \mathrm{MHz}, \mathrm{CDCl}_{3}\right): \delta 5.85(\mathrm{~s}, 1 \mathrm{H}), 4.94$ (s, 1H), 4.73 (s, 1H), 2.27 (t, $J=6.6 \mathrm{~Hz}, 2 \mathrm{H}), 2.19(\mathrm{dd}, J=18.1,13.5 \mathrm{~Hz}, 2 \mathrm{H}), 1.86-1.72(\mathrm{~m}$, $5 \mathrm{H}), 1.69-1.57(\mathrm{~m}, 2 \mathrm{H}), 1.56-1.36(\mathrm{~m}, 2 \mathrm{H}), 1.36-1.13(\mathrm{~m}, 6 \mathrm{H}), 0.87(\mathrm{t}, J=7.2 \mathrm{~Hz}, 3 \mathrm{H}) .{ }^{13} \mathrm{C}$ $\operatorname{NMR}\left(100 \mathrm{MHz}, \mathrm{CDCl}_{3}\right): \delta 172.0,141.0,116.6,57.1,47.7,41.0,32.2,31.2,31.1,25.2,23.4,22.7$, 17.4, 14.1. HRMS (ESI) calcd for $\mathrm{C}_{14} \mathrm{H}_{25} \mathrm{NONa}(\mathrm{M}+\mathrm{Na})^{+} 246.1828$, found 246.1865 .

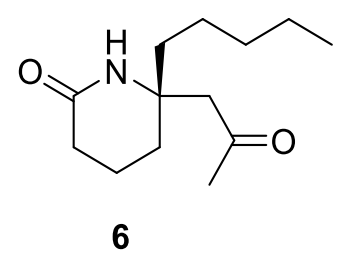

(+)-Adalinine (6). To a vial containing lactam $4(19 \mathrm{mg}, 0.085 \mathrm{mmol})$ and a magnetic stir bar was added 1,4-dioxane $(2.1 \mathrm{~mL})$ and deionized water $(2.1 \mathrm{~mL})$. N-methylmorpholine $\mathrm{N}$-oxide $(87.2 \mathrm{mg}, 0.74 \mathrm{mmol})$ and sodium periodate $(127.4 \mathrm{mg}, 0.60 \mathrm{mmol})$ were added, respectively, followed by osmium tetroxide $(28.4 \mu \mathrm{L}, 1 \% \mathrm{in} \mathrm{wt})$. The reaction was stirred at room temperature for $18 \mathrm{~h}$, then diluted with DCM followed by addition of a saturated aqueous solution of sodium thiosulfate. The organic layer was separated, and the aqueous layer was extracted 3 times using DCM. The combined organic layers were dried over sodium sulfate, filtered, and stripped of solvent under reduced pressure. The crude material was purified via flash column chromatography on silica gel (50\% EtOAc/hexanes eluted impurity, 50\% $\mathrm{MeOH} / \mathrm{DCM}$ flush eluted product) to afford (+)-adalinine $(18.6 \mathrm{mg}, 0.083 \mathrm{mmol})$ in $97 \%$ yield as an oil. $\mathrm{R}_{\mathrm{f}}=0.48(10 \% \mathrm{MeOH} / \mathrm{DCM}$, DNPH stain). $[\alpha]^{22}{ }_{\mathrm{D}}=+18.9(\mathrm{c}=0.13, \mathrm{DCM}) .{ }^{1} \mathrm{H}$ NMR $\left(400 \mathrm{MHz}, \mathrm{CDCl}_{3}\right) \delta 6.66(\mathrm{~s}, 1 \mathrm{H}), 2.69$ $(\mathrm{d}, J=17.7 \mathrm{~Hz}, 1 \mathrm{H}), 2.62(\mathrm{~d}, J=17.8 \mathrm{~Hz}, 1 \mathrm{H}), 2.37-2.16(\mathrm{~m}, 2 \mathrm{H}), 2.12(\mathrm{~s}, 3 \mathrm{H}), 1.88-1.45(\mathrm{~m}$, $6 \mathrm{H}), 1.36-1.04(\mathrm{~m}, 6 \mathrm{H}), 0.85(\mathrm{t}, J=6.8 \mathrm{~Hz}, 3 \mathrm{H})$; HRMS (ESI) calcd for $\mathrm{C}_{13} \mathrm{H}_{23} \mathrm{NO}_{2}(\mathrm{M}+\mathrm{H})+$ 226.1802 , found 226.1801 . 


\section{6. ${ }^{31}$ P-NMR Studies}

\subsection{Detection of 3-34 by ${ }^{31} \mathrm{P}-\mathrm{NMR}$}

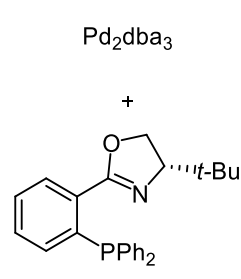

L1

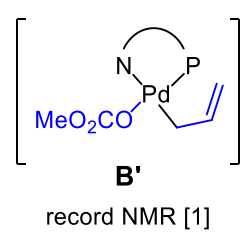

$1 \mathrm{a}$ $\underset{\text { THF }-\mathrm{d}_{8}, 25^{\circ} \mathrm{C}}{\stackrel{\text { NaHMDS }}{\longrightarrow}}$

Figure S1. Reaction scheme for detection of C'.

Under an argon atmosphere in a glovebox was added $\mathrm{Pd}_{2} \mathrm{dba}_{3}(3.0 \mathrm{mg}, 3.3 \mu \mathrm{mol}, 5.25 \mathrm{~mol} \%)$ and (S)-t-Bu-PHOX, L2 (3.3 mg, $8.5 \mu \mathrm{mol}, 13.5 \mathrm{~mol} \%)$ to an oven-dried flask containing a magnetic stirrer. The flask was capped with a rubber septum. To a second flask was added NaHMDS (12.1 $\mathrm{mg}, 62.8 \mu \mathrm{mol}, 1$ equiv). This flask was capped with a rubber septum followed by removal of both vessels from the glove box. Both flasks were then placed under a nitrogen atmosphere. Dry, degassed, THF- $\mathrm{d}_{8}(0.5 \mathrm{~mL})$ was added to the flask containing the catalyst and ligand. This mixture was stirred for $0.5 \mathrm{~h}$ at room temperature followed by the neat addition of allyl methyl carbonate (6.3 uL, $55.1 \mu \mathrm{mol}, 1$ equiv). The mixture was stirred for 1 minute and then transferred to an ovendried J-Young tube under a nitrogen environment via syringe through a screw-cap with a pierceable septum top. The first ${ }^{31} \mathrm{P}$ NMR was acquired at this point and revealed two signals (31.74 ppm and -5.95). The J-Young tube was then placed back under a nitrogen atmosphere. To the flask containing NaHMDS was added $0.2 \mathrm{~mL}$ THF- $\mathrm{d}_{8}$. To a vial containing carboxylic acid 1a (9.8 mg, $62.8 \mu \mathrm{mol}, 1$ equiv) under a nitrogen atmosphere was added $0.3 \mathrm{~mL}$ THF-d8 followed by the transfer of acid 1a to flask containing NaHMDS solution. The NaHMDS/1a mixture was swirled vigorously followed by transfer to the J-Young tube. The contents of the J-Young tube 
were shaken and the second ${ }^{31} \mathrm{P}$ NMR was acquired at this point, revealing signals at $30.5 \mathrm{ppm}$, $27.1 \mathrm{ppm}$, and $-5.95 \mathrm{ppm}$ corresponding to $\mathbf{C}^{\prime}$, the phosphine oxide of ligand $\mathbf{L} \mathbf{2}$, and free ligand L2, respectively, which matched previously reported data. ${ }^{7}$
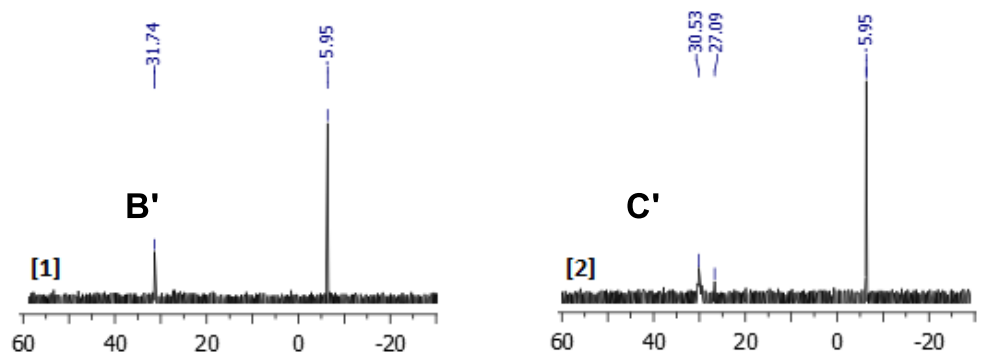

\section{References}

(1) Giedt, R. J.; Sprachman, M. M.; Yang, K. S.; Weissleder, R. Imaging Cellular Distribution of Bcl Inhibitors Using Small Molecule Drug Conjugates. Bioconjugate Chem. 2014, 25, 11, 20812085.

(2) Shibatomi, K.; Kitahara, K.; Sasaki, N; Kawasaki, Y.; Fujisawa, I.; Iwasa, S. Enantioselective decarboxylative chlorination of $\beta$-ketocarboxylic acids. Nat. Commun. 2017, 8, 15600.

(3) Bunnage, M E.; Davies, S. G.; Parkin, R. M.; Roberts, B. M.; Smith, A. D.; Witheyb, J. M. Kinetic resolution of tert-butyl (RS)-3-alkylcyclopentene-1-carboxylates for the synthesis of homochiral 3-alkyl-cispentacin and 3-alkyl-transpentacin derivatives. Org. Biomol. Chem. 2004, 2 , 3337-3354.

(4) Palomo, C.; Oiarbide, M.; García' J. M.; Bañuelos, P.; Odriozola, J. M.; Linden, R. A. Catalytic Michael Reactions of Ketoesters with a Camphor-Derived Acrylate Equivalent: Stereoselective Access to All-Carbon Quaternary Centers. Org. Lett. 2008, 10, 13, 2637-2640.

(5) Chen, H-F.; Jia, X.; Yu, Y-G.; Qian, Q.; Gong, H-G. Nickel-Catalyzed Reductive Allylation of Tertiary Alkyl Halides with Allylic Carbonates. Angew. Chem., Int. Ed. 2017, 56, 13103-13106.

(6) Behenna, D. C.; Stoltz, B. M. The Enantioselective Tsuji Allylation. J. Am. Chem. Soc. 2004, $126,15044-15045$.

(7) Sherden, N. H.; Behenna, D. C.; Virgil, S. C.; Stoltz, B. M. Unusual Allylpalladium Carboxylate Complexes: Identification of the Resting State of Catalytic Enantioselective Decarboxylative Allylic Alkylation Reactions of Ketones. Angew. Chem. Int. Ed. 2009, 48, 6840. 


\section{NMR}

' $\mathrm{HNMR}(500 \mathrm{MHz}, \mathrm{CDCl} 3)$

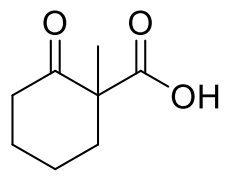

$1 a$

$1 \int$

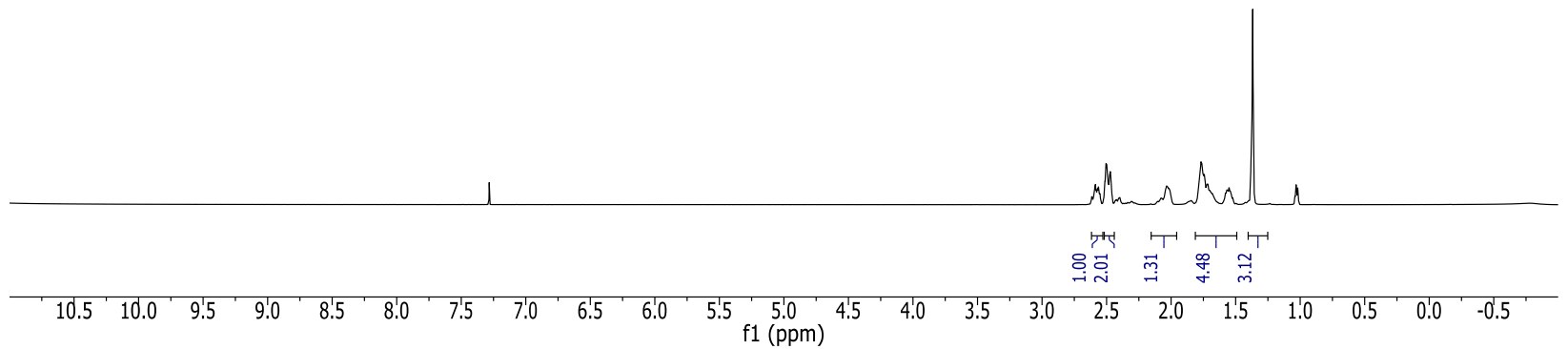

13C NMR (125 MHz, CDCl3)

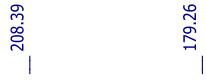

怨

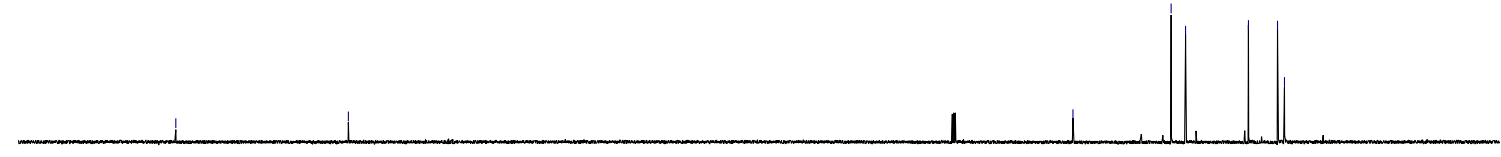

\begin{tabular}{lllllllllllllllllllllllllllllll}
\hline 230 & 220 & 210 & 200 & 190 & 180 & 170 & 160 & 150 & 140 & 130 & 120 & 110 & 100 & 90 & 80 & 70 & 60 & 50 & 40 & 30 & 20 & 10 & 0 & -10
\end{tabular} 


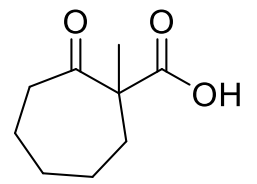

$1 b$

${ }^{1} \mathrm{H} \mathrm{NMR}(500 \mathrm{MHz}, \mathrm{CDCl} 3)$
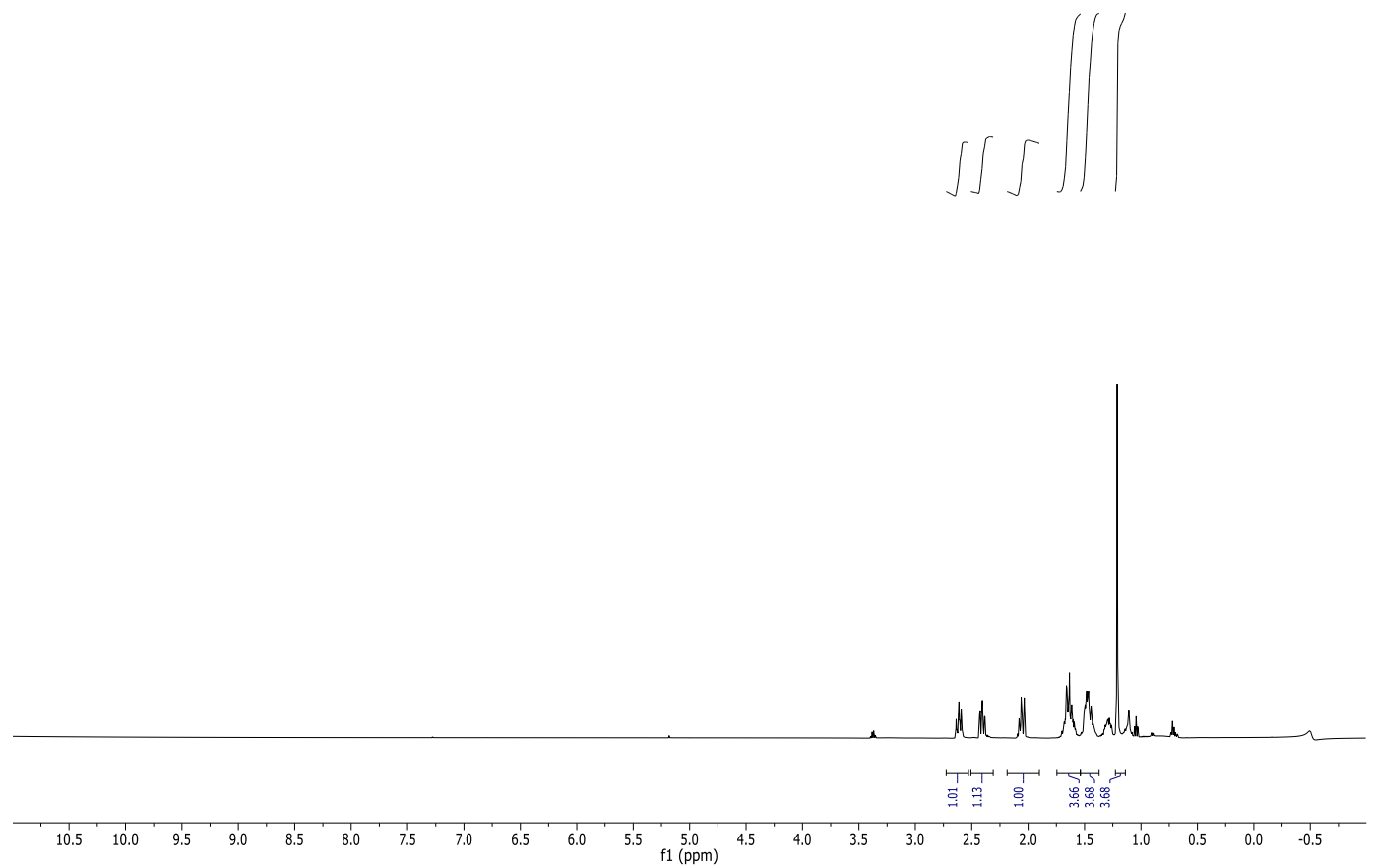

13C NMR (125 MHz, CDCl3

弪
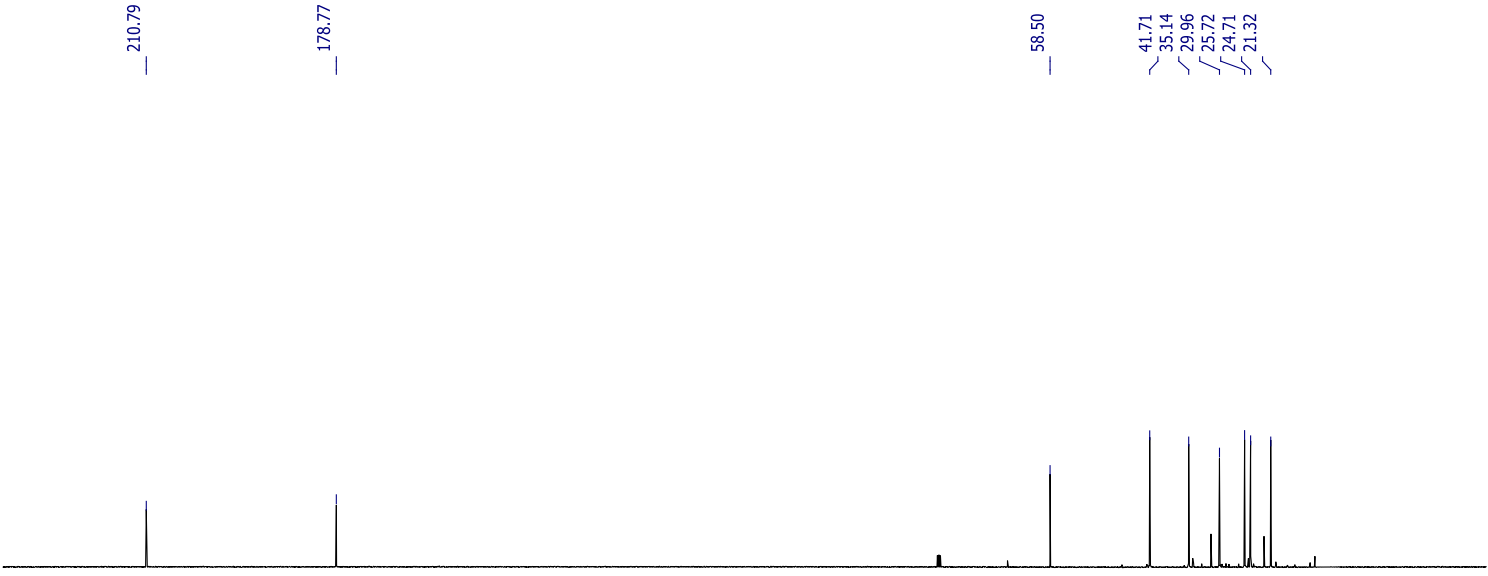

\begin{tabular}{llllllllllllllllllllllllllllllll}
\hline 230 & 220 & 210 & 200 & 190 & 180 & 170 & 160 & 150 & 140 & 130 & 120 & 110 & 100 & 90 & 80 & 70 & 60 & 50 & 40 & 30 & 20 & 10 & 0 & -10
\end{tabular} 
'H NMR (500 MHz,CDCl3)<smiles>CC1(C)CCC(C(=O)O)(C(=O)O)C(=O)C1</smiles>

$1 \mathrm{~h}$
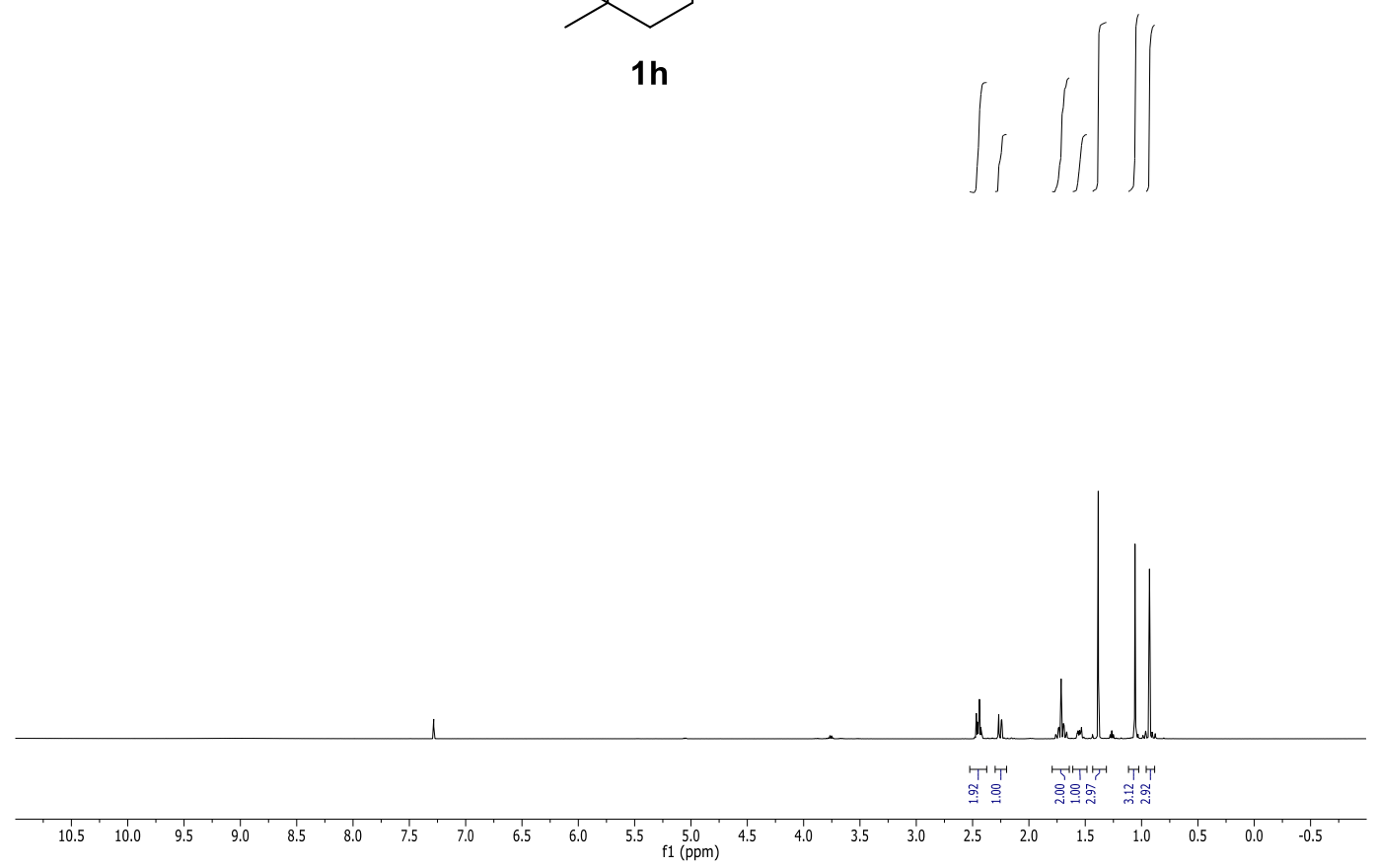

${ }^{13} \mathrm{C} \mathrm{NMR}\left(125 \mathrm{MHz}_{1} \mathrm{CDCl}_{3}\right)$

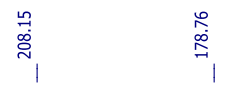

कo

11 lit।

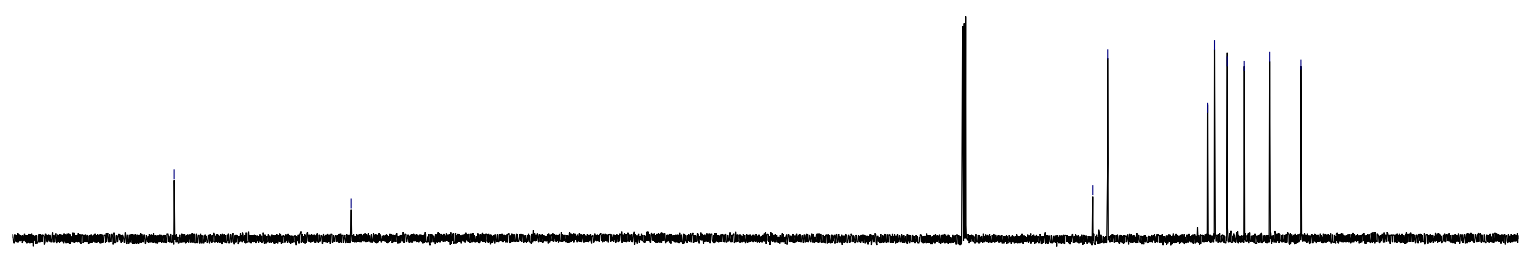

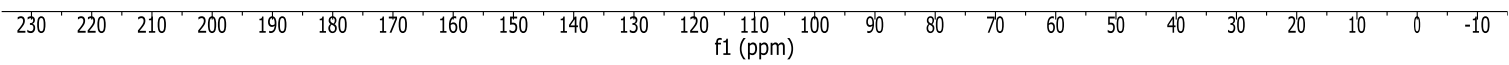




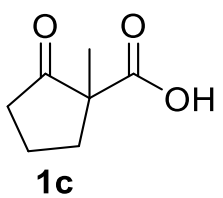

H NMR (500 MHz, Chloroform- d)

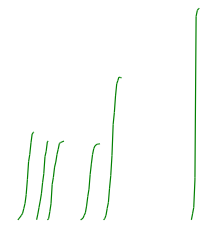

Whith

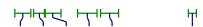

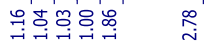

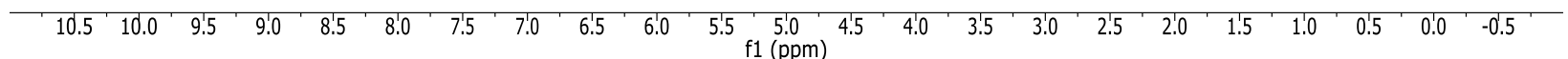

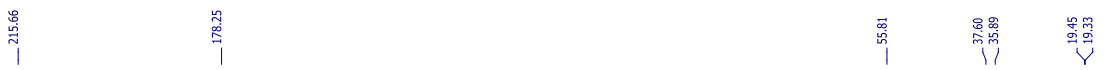

$\left.{ }^{13} \mathrm{C} \mathrm{NMR} \mathrm{(125} \mathrm{MHz}, \mathrm{CDCl}_{3}\right)$

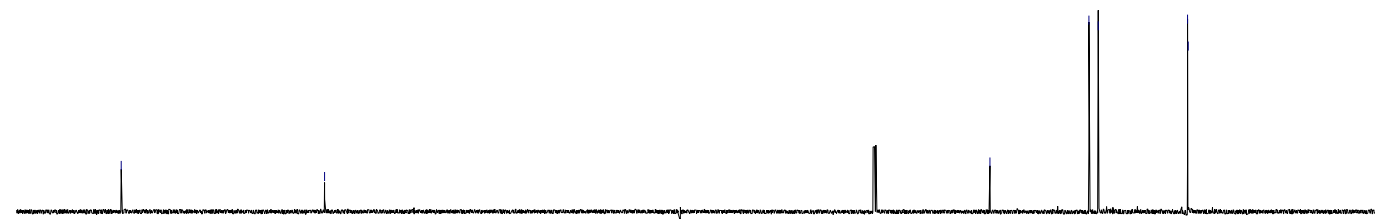

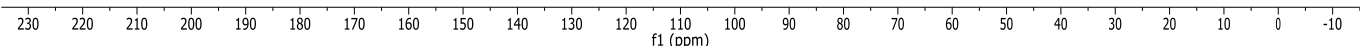




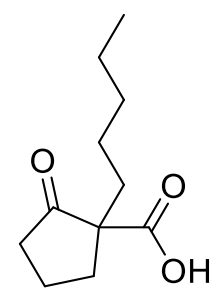

$1 q$

' H NMR (300 MHz, Chloroform- $d$ )

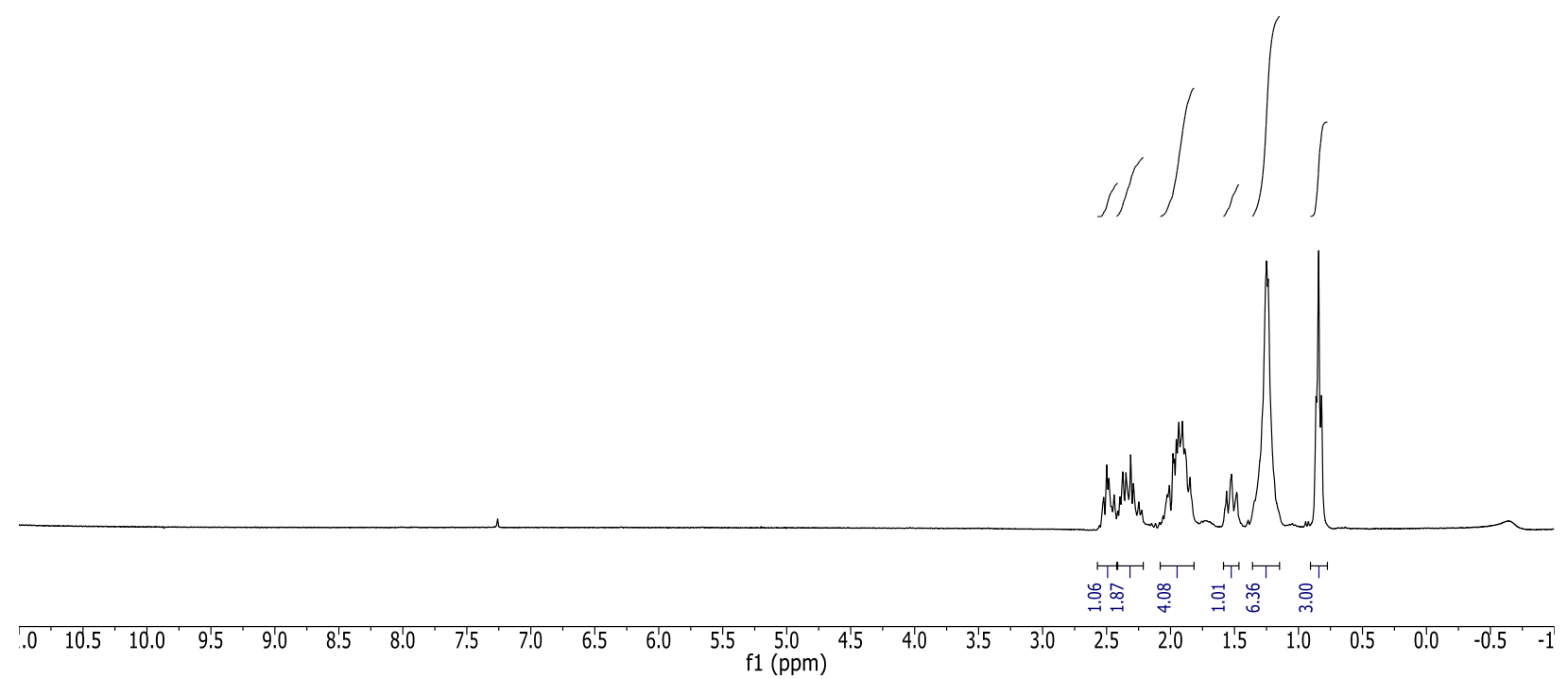

"C NMR (125MHz, CDCl,

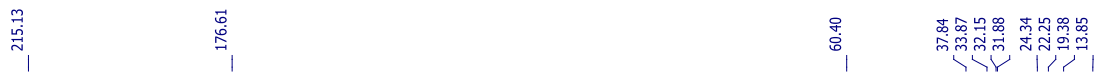

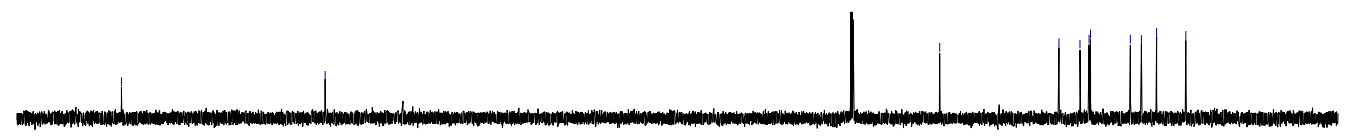

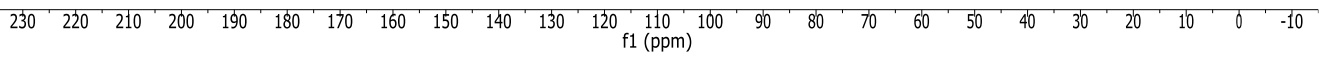




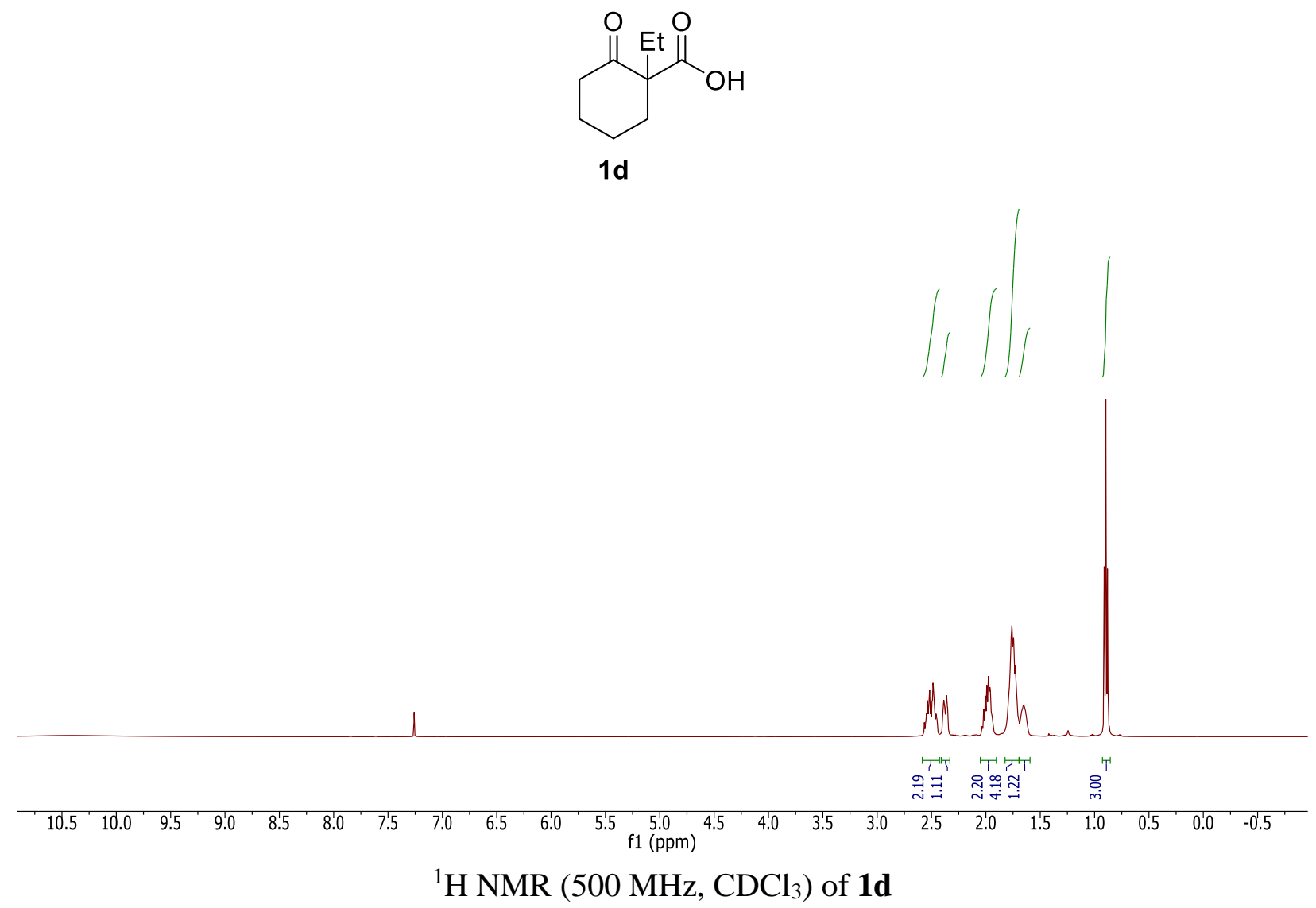

${ }^{13} \mathrm{CNMR}\left(125 \mathrm{MHz}, \mathrm{CDCl}_{3}\right.$
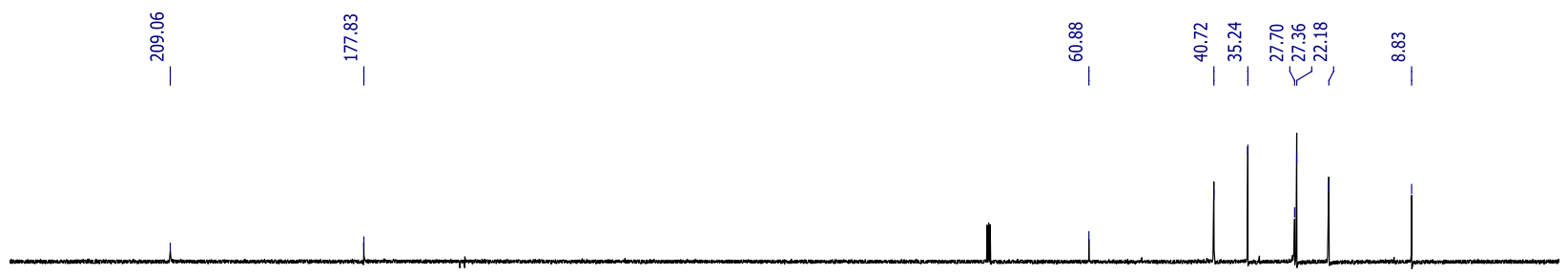

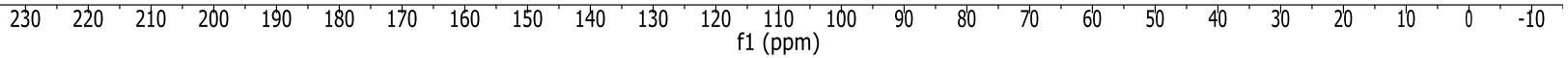




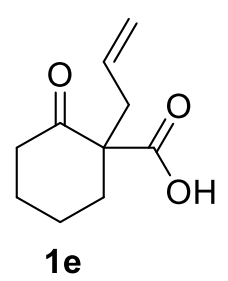

' $\mathrm{H}$ NMR (500 MHz, CDCl3)
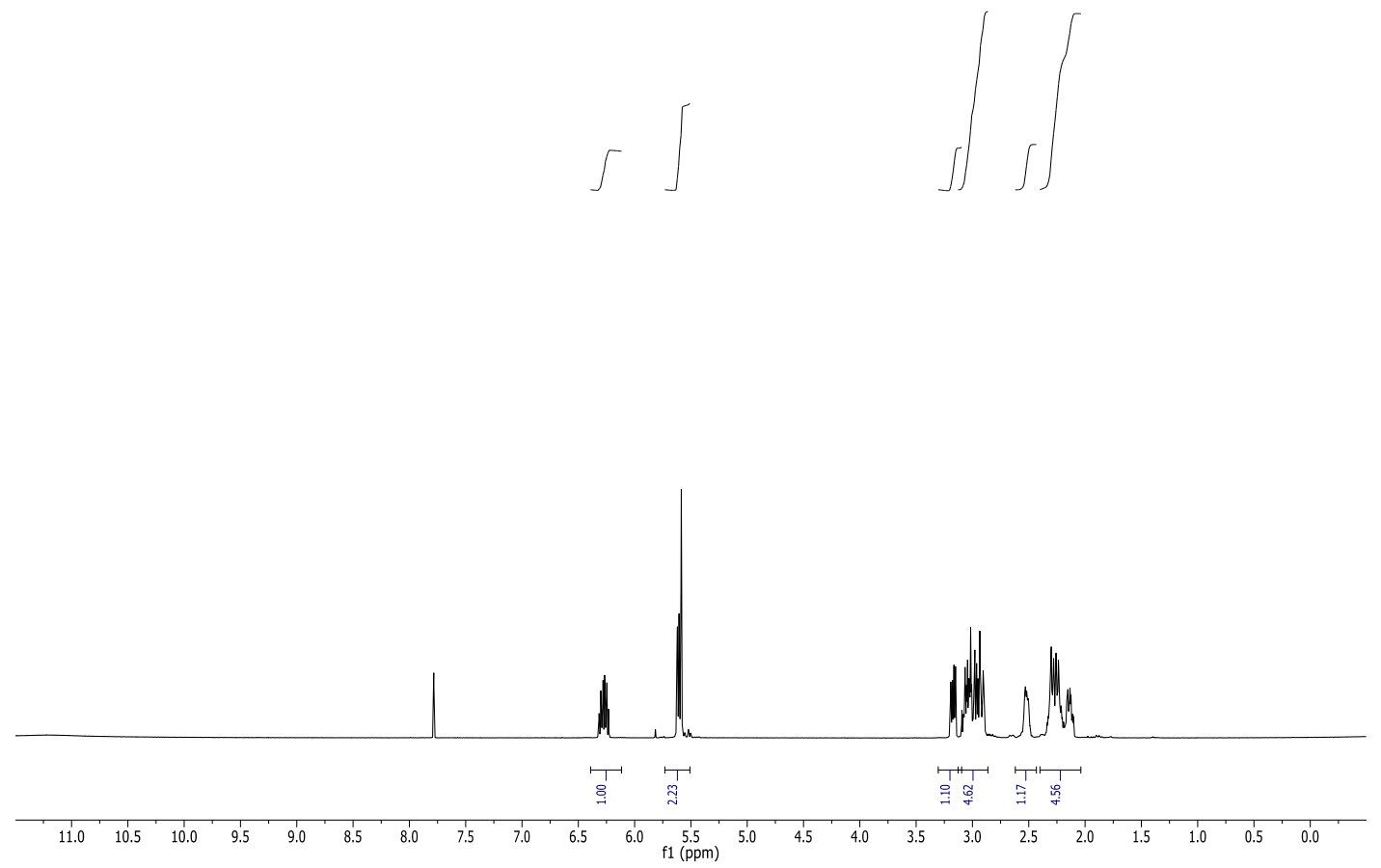

" $\mathrm{C} \mathrm{NMR}(125 \mathrm{MHz}, \mathrm{CDCl}$

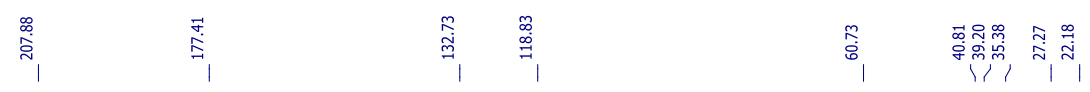

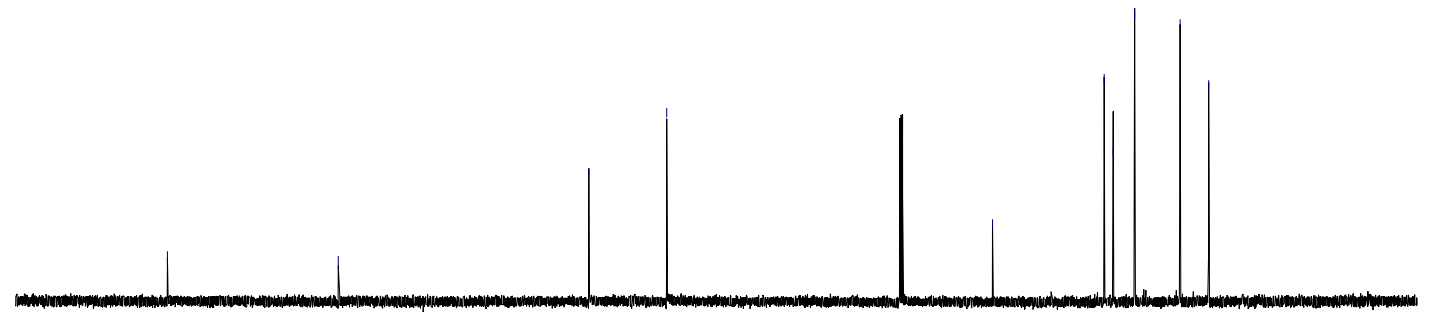

\begin{tabular}{l|l|l|llllllllllllllllllllllllll}
230 & 220 & 210 & 200 & 190 & 180 & 170 & 160 & 150 & 140 & 130 & 120 & $\begin{array}{c}110 \\
\mathrm{f}(\mathrm{ppm})\end{array}$ & 100 & 90 & 80 & 70 & 60 & 50 & 40 & 30 & 20 & 10 & 0 & -10
\end{tabular} 


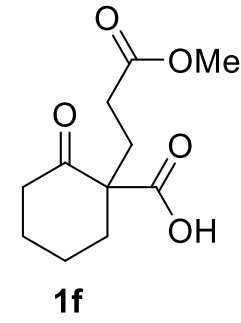

' $\mathrm{H} \mathrm{NMR}(500 \mathrm{MHz}, \mathrm{CDCl} 3)$
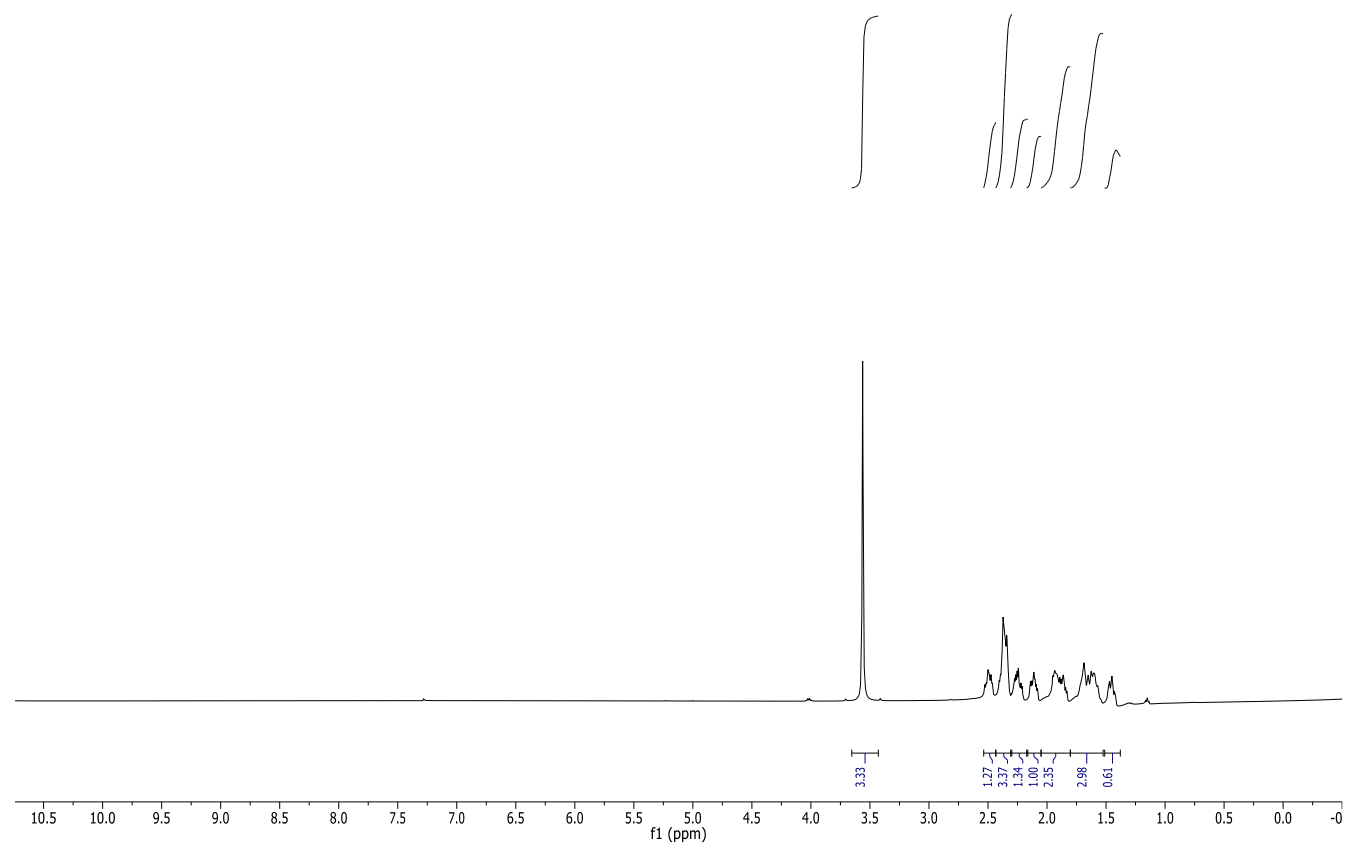

${ }^{13} \mathrm{CNMR}(125 \mathrm{MHz}, \mathrm{CDCl}$

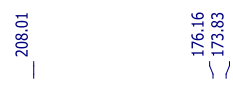
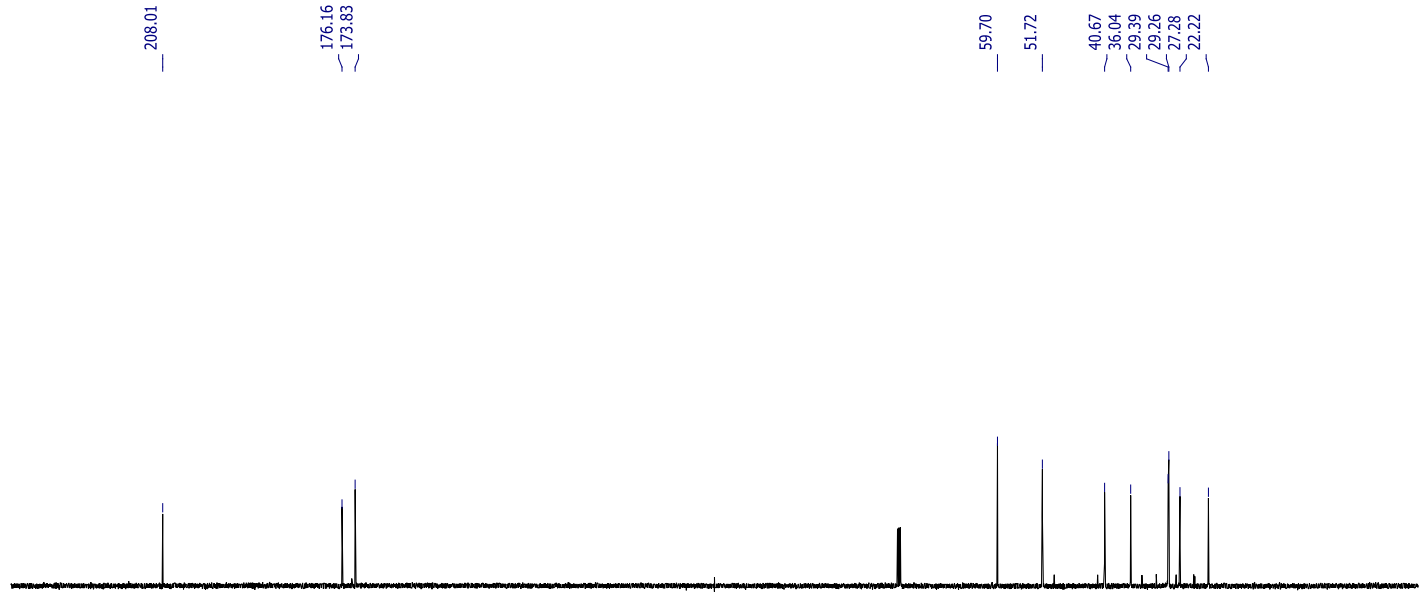

\begin{tabular}{llllllllllllllllllllllllllllll}
\hline 230 & 220 & 210 & 200 & 190 & 180 & 170 & 160 & 150 & 140 & 130 & 120 & 110 & 100 & 90 & 80 & 70 & 60 & 50 & 40 & 30 & 20 & 10 & 0 & -10
\end{tabular} 


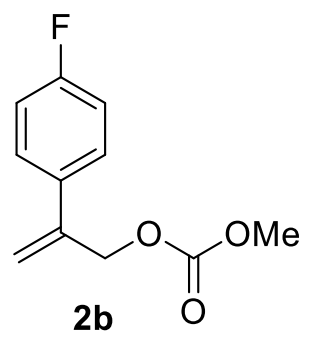

' H NMR (500 MHz, CDCl3)
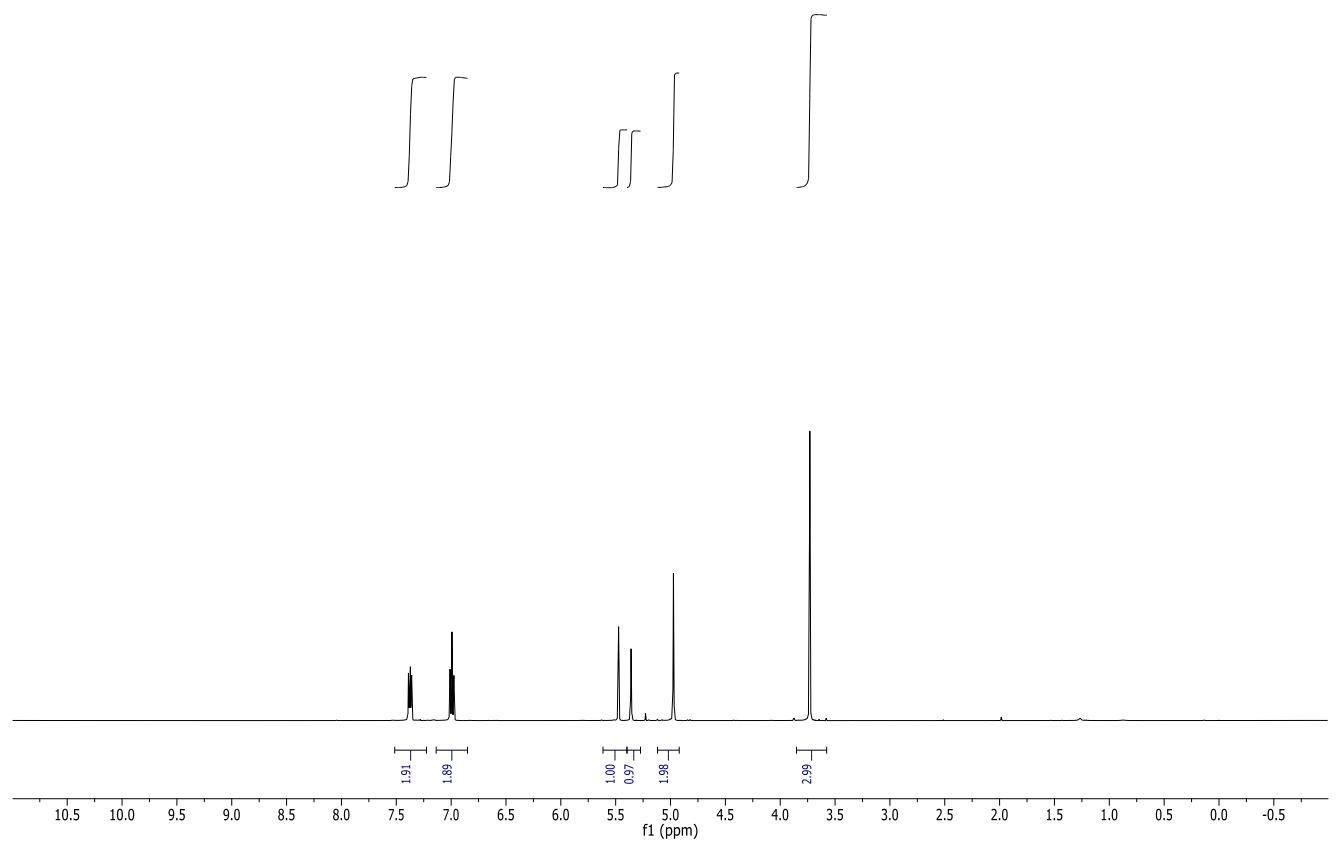

${ }^{15} \mathrm{C} \mathrm{NMR}\left(125 \mathrm{MHz}, \mathrm{CDCl}_{3}\right)$
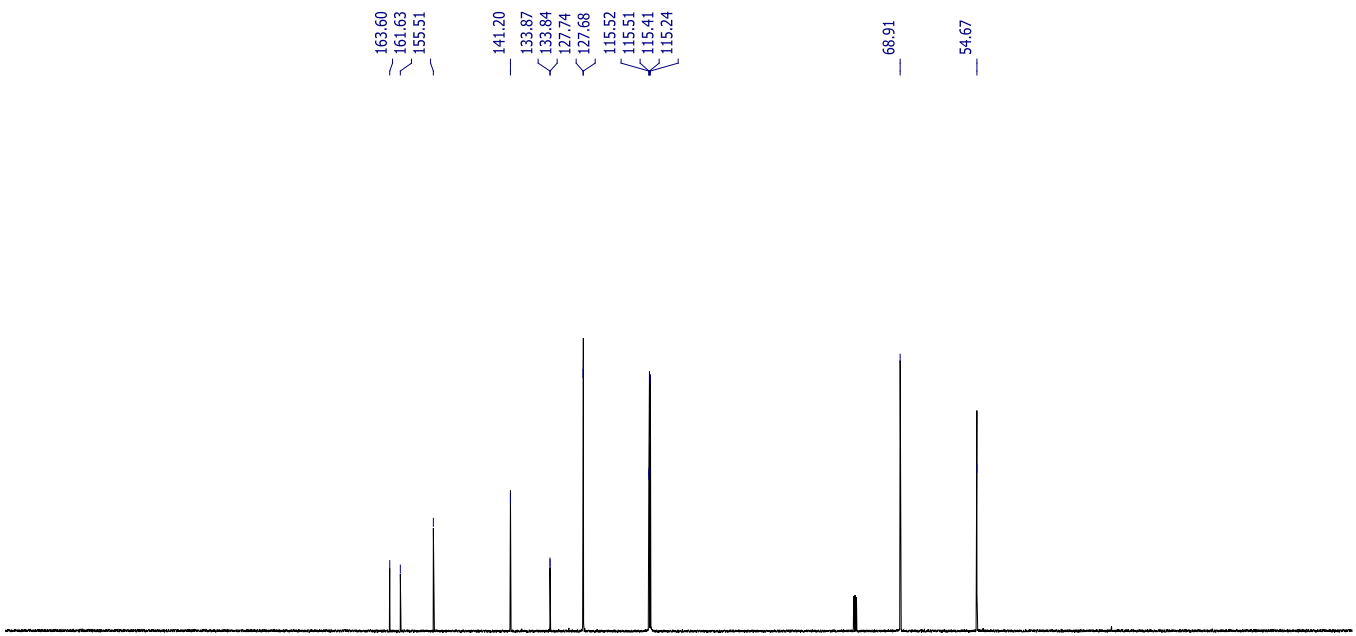

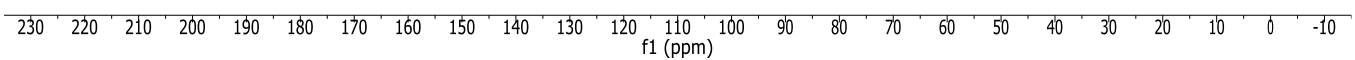




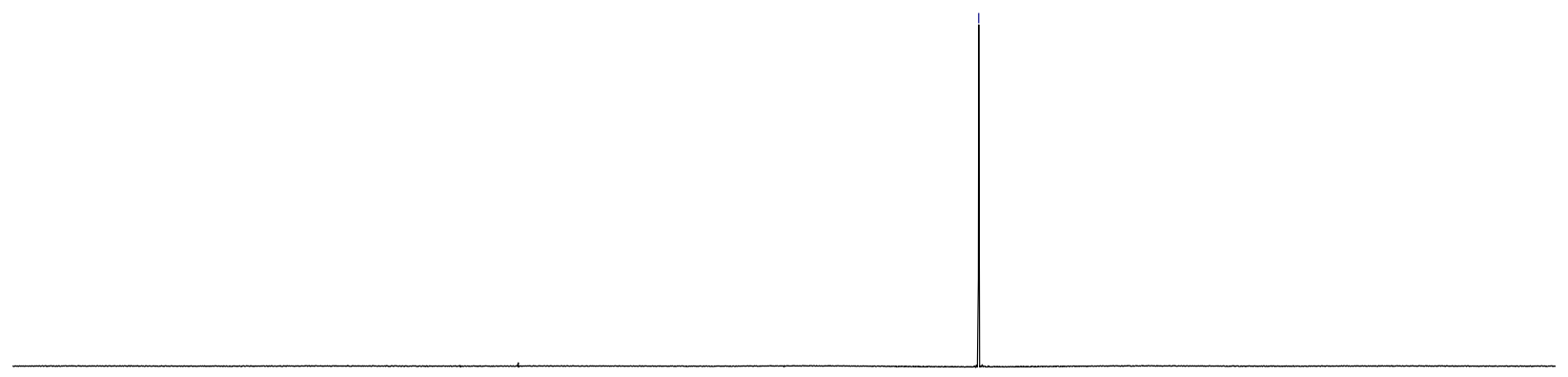

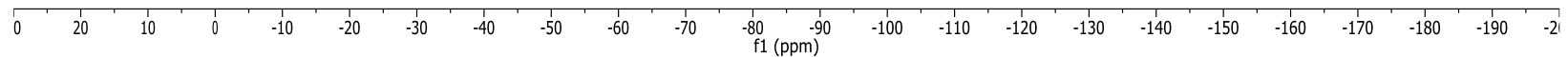




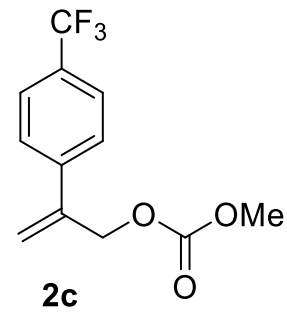

' H NMR (500 MHz, CDC13)
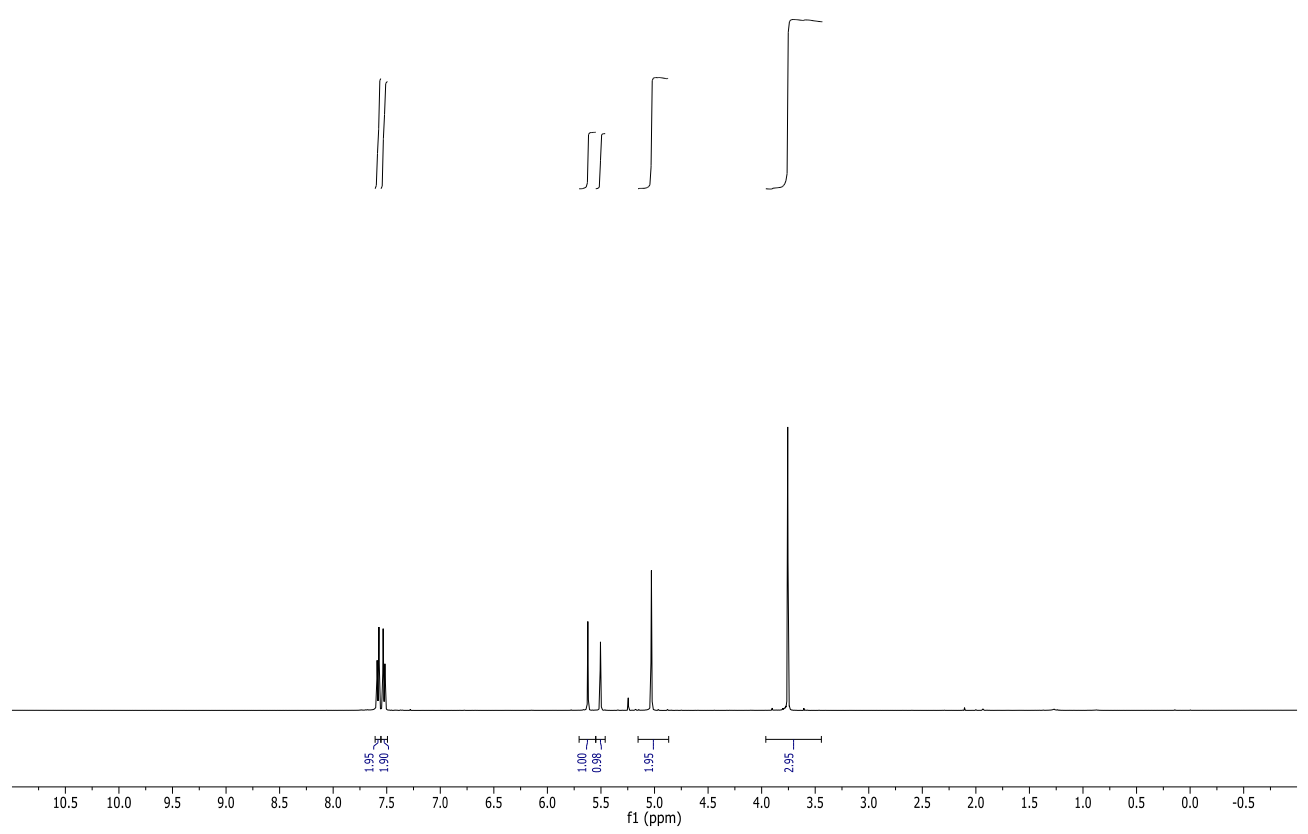

${ }^{3} \mathrm{CNMR}(125 \mathrm{MHz}, \mathrm{CDCl}$
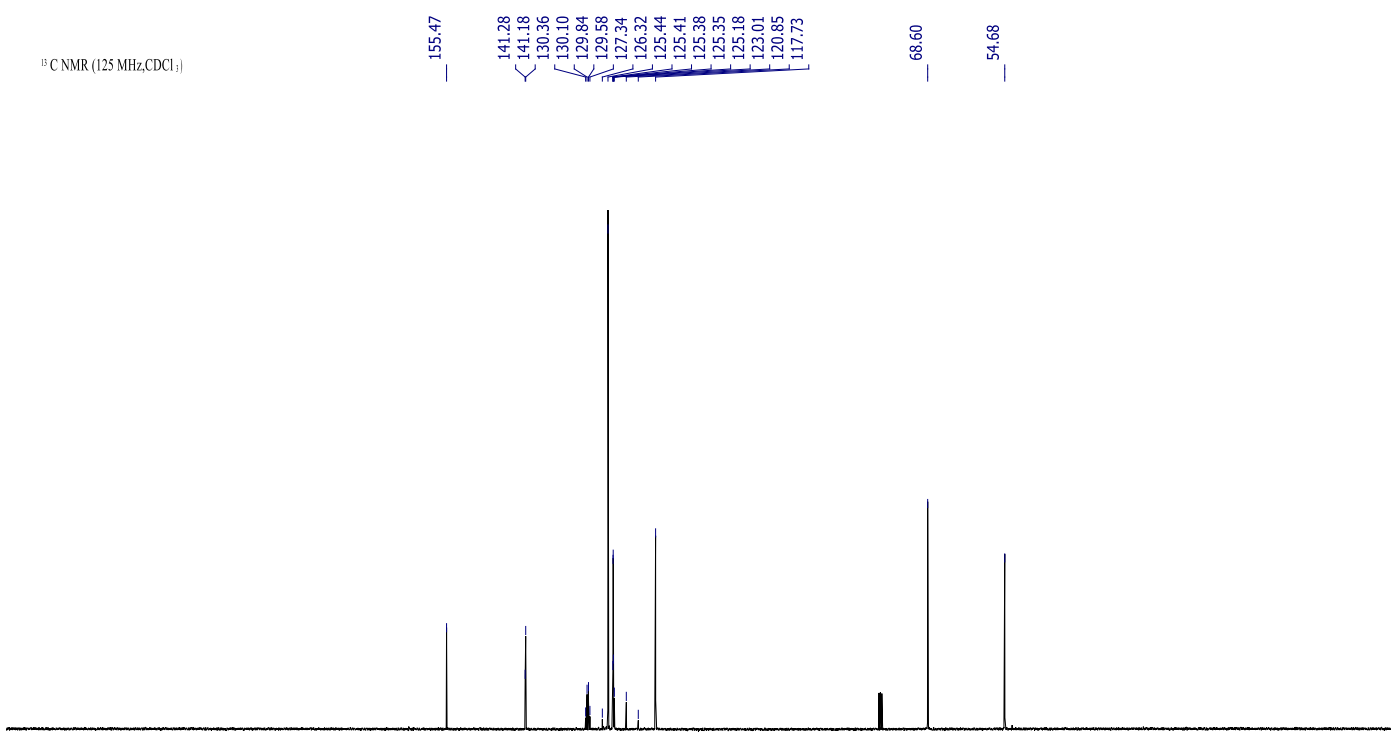

\begin{tabular}{lllllllllllllllllllllllllll}
\hline 230 & 220 & 210 & 200 & 190 & 180 & 170 & 160 & 150 & 140 & 130 & 120 & 110 & 100 & 90 & 80 & 70 & 60 & 50 & 40 & 30 & 20 & 10 & 0 & -10
\end{tabular} 
${ }^{19} \mathrm{~F} \mathrm{NMR}\left(300 \mathrm{MHz}, \mathrm{CDCl}_{3}\right)$

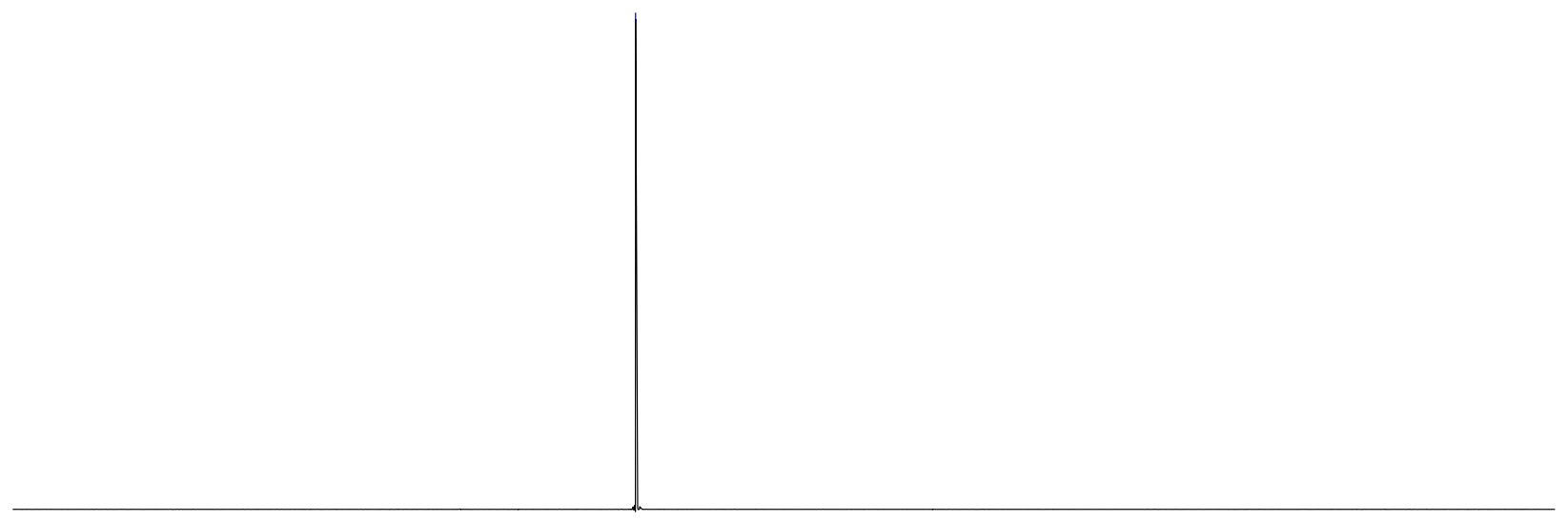

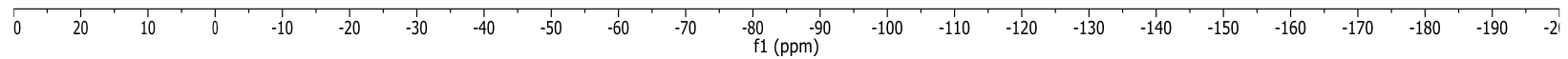




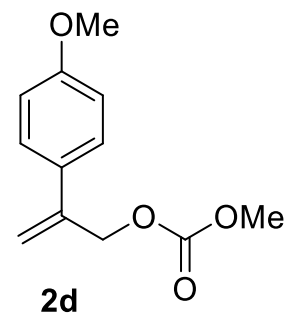

' H NMR (500 MHz, CDCl3)
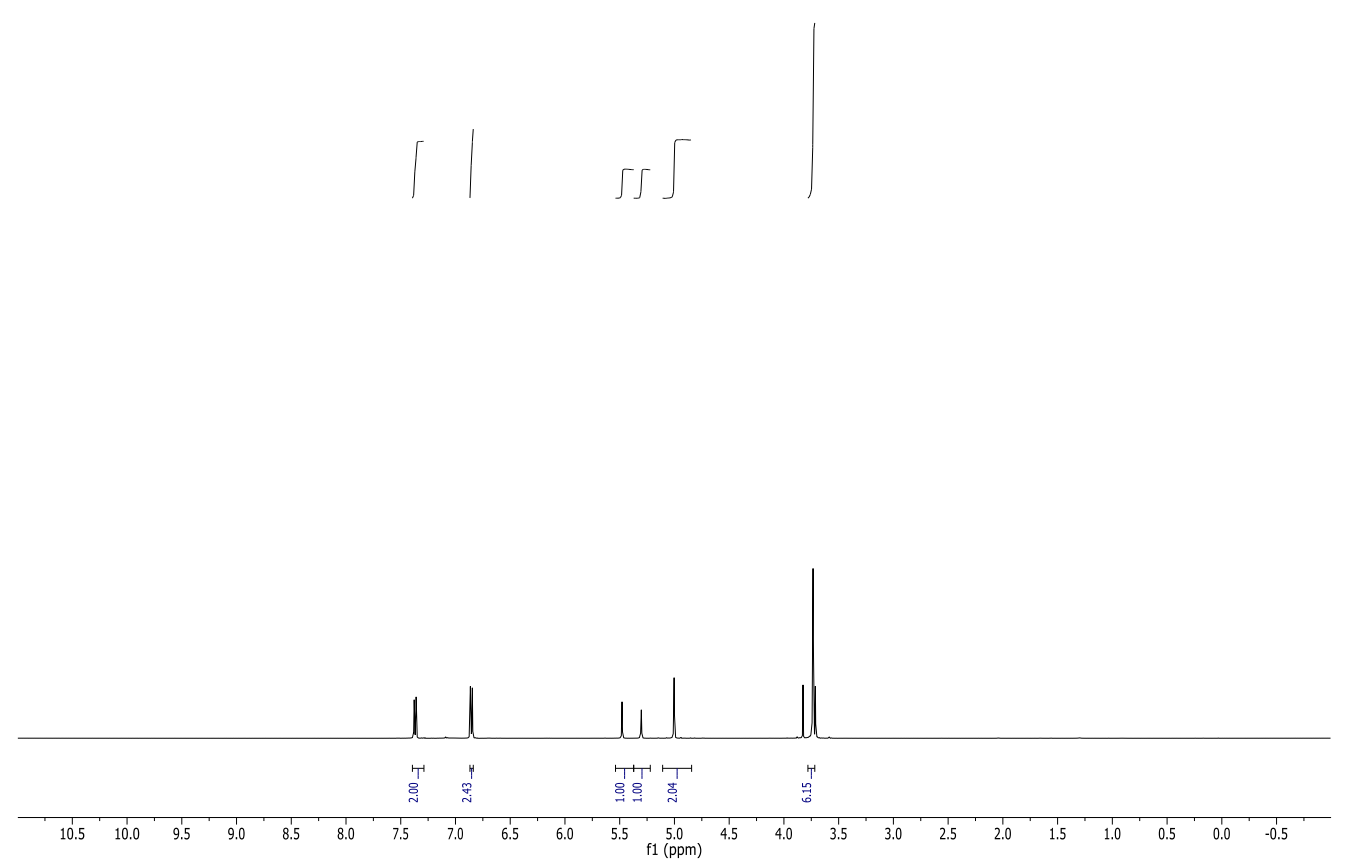

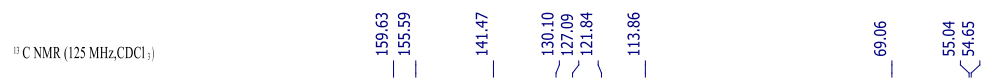

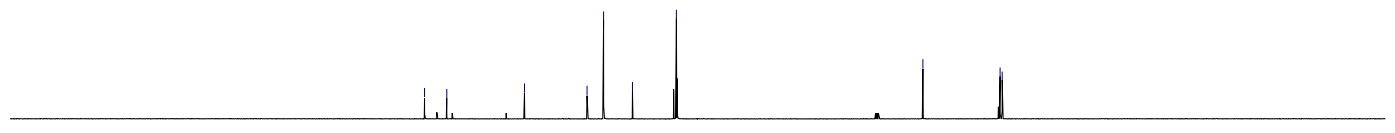

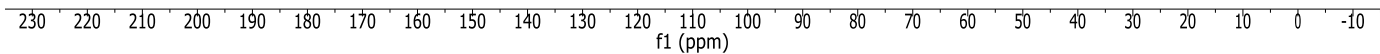




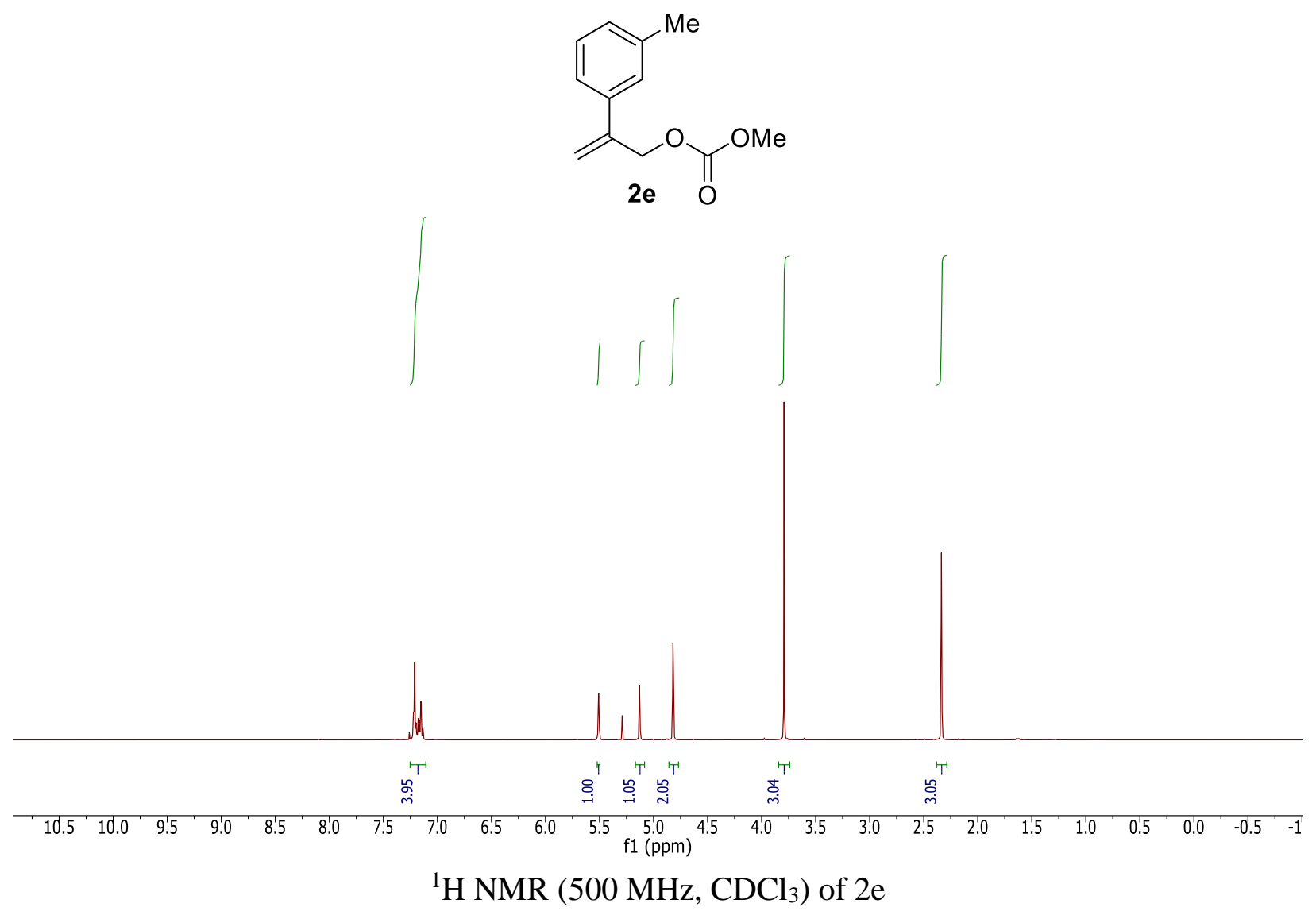

"C CNMR (125MHz, CDCl $) \quad$ |

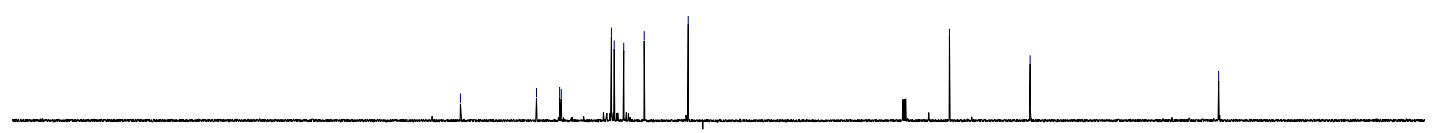

\begin{tabular}{lllllllllllllllllllllllll}
\hline 230 & 220 & 210 & 200 & 190 & 180 & 170 & 160 & 150 & 140 & 130 & 120 & 110 & 100 & 90 & 80 & 70 & 60 & 50 & 40 & 30 & 20 & 10 & 0 & -10
\end{tabular} 

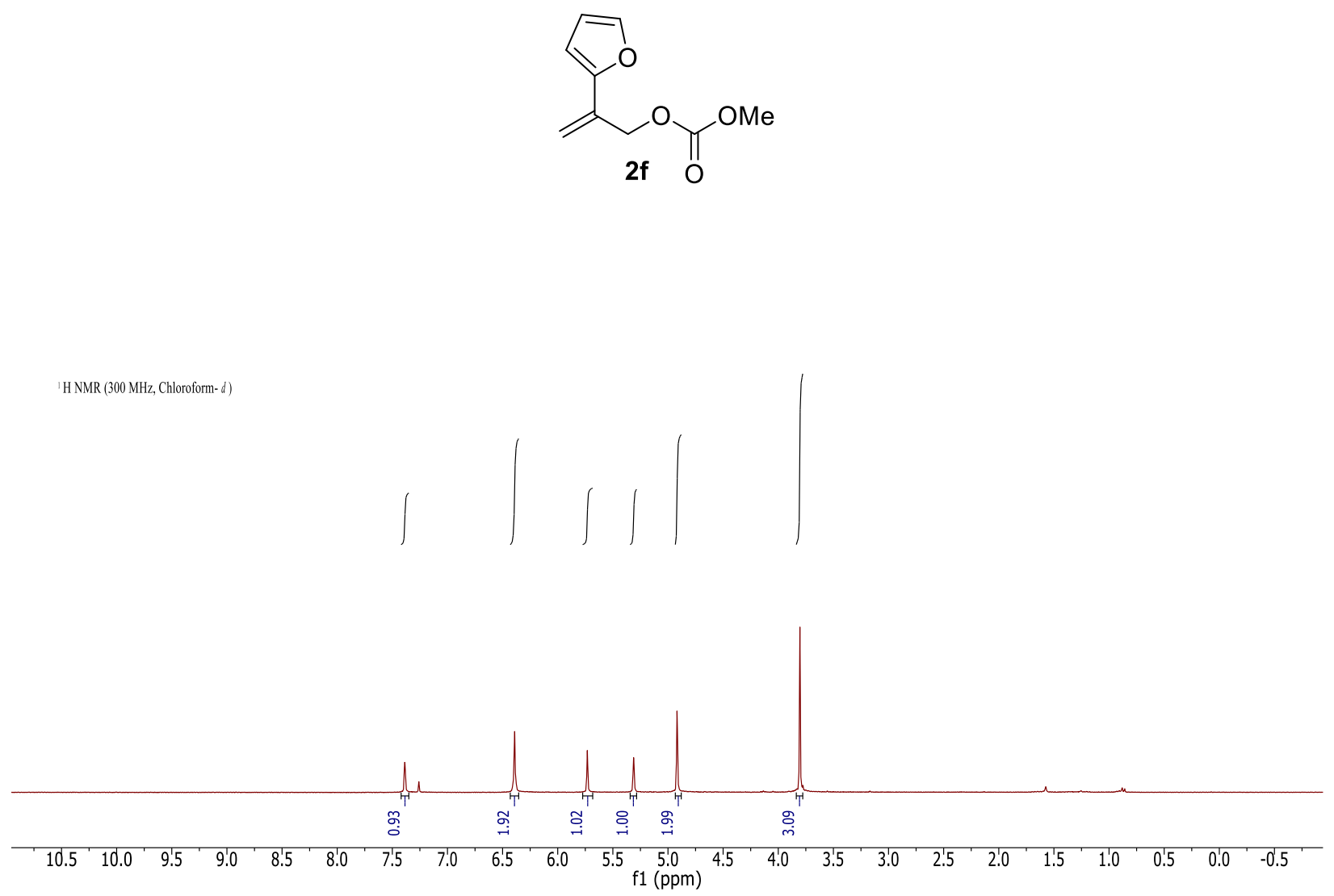

${ }^{3} \mathrm{CNMR}\left(100 \mathrm{MHz}, \mathrm{CDCl}_{3}\right)$
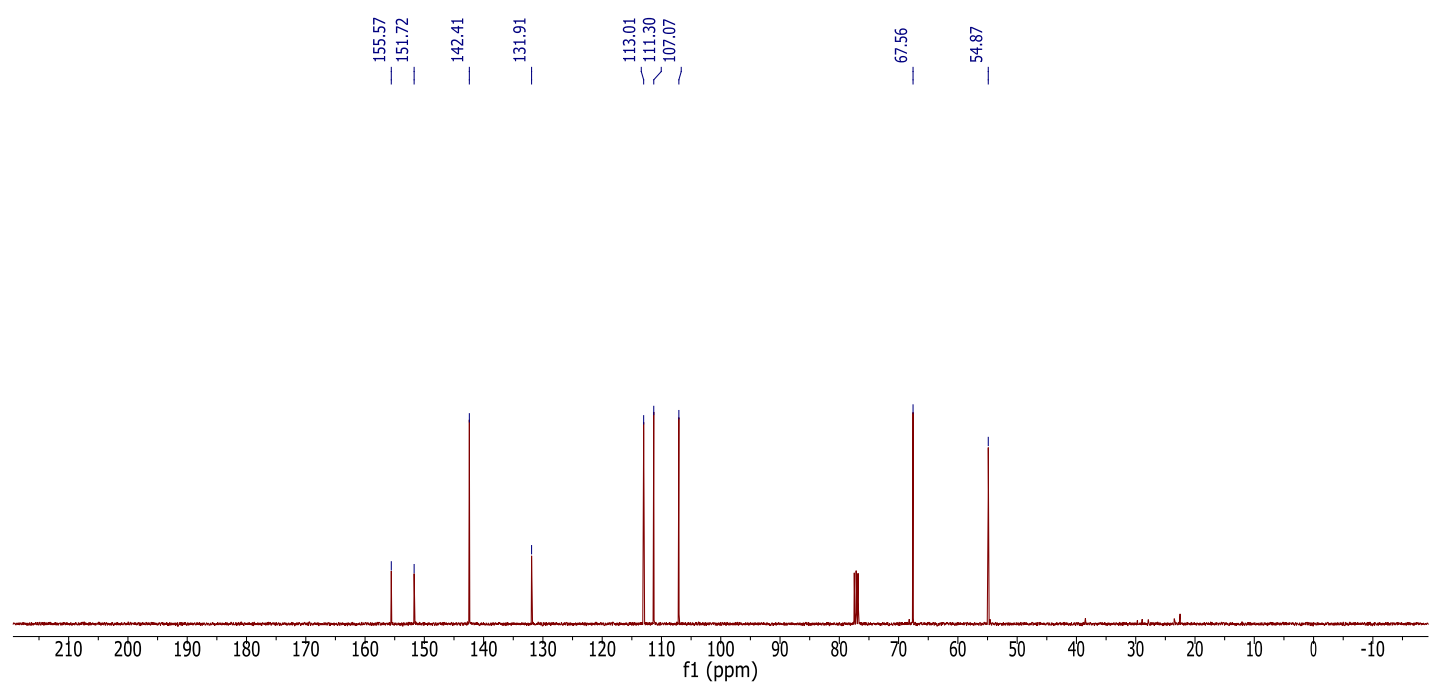

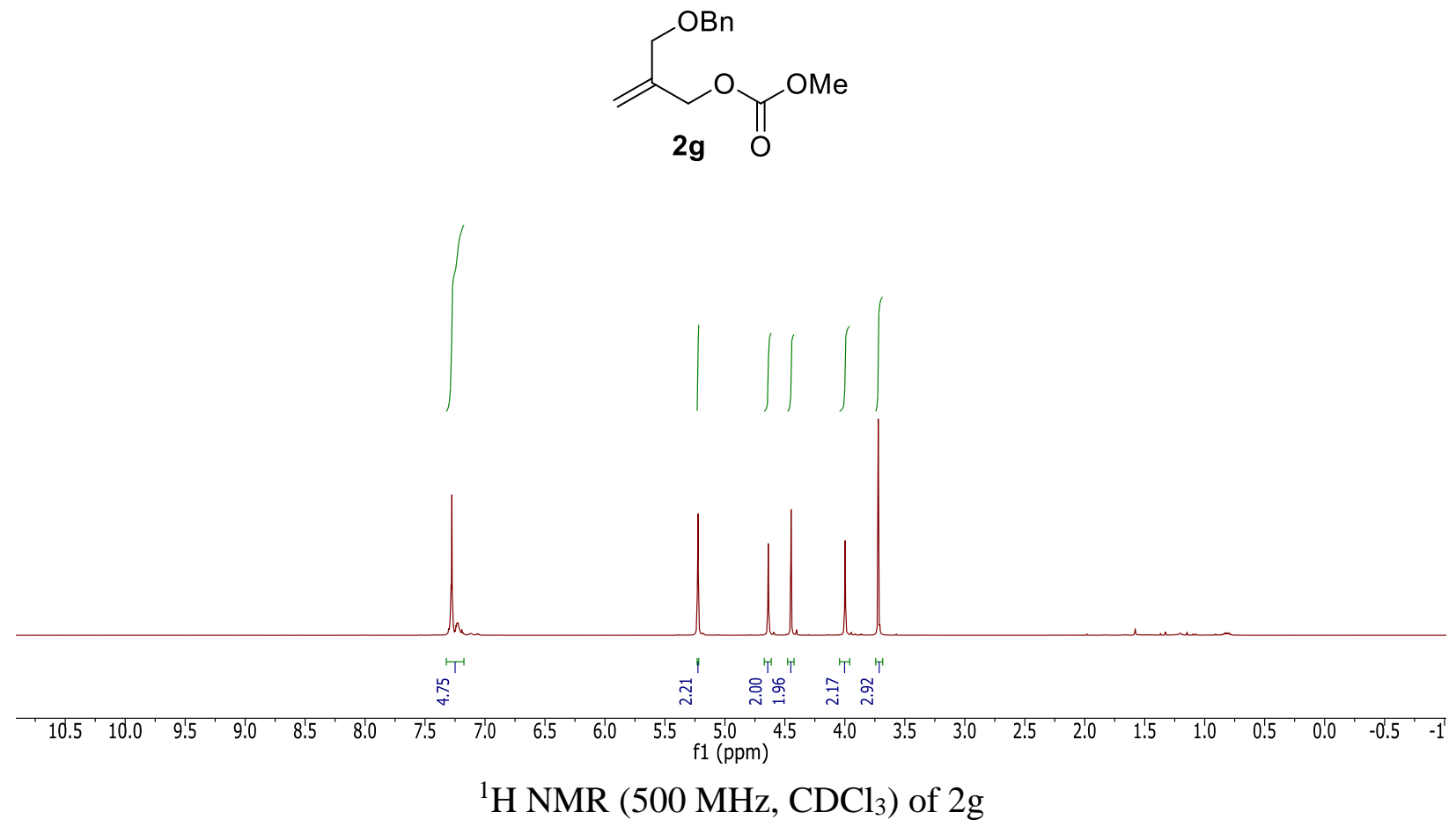

${ }^{13} \mathrm{CNMR}\left(150 \mathrm{MHz}, \mathrm{CDCl}_{3}\right)$
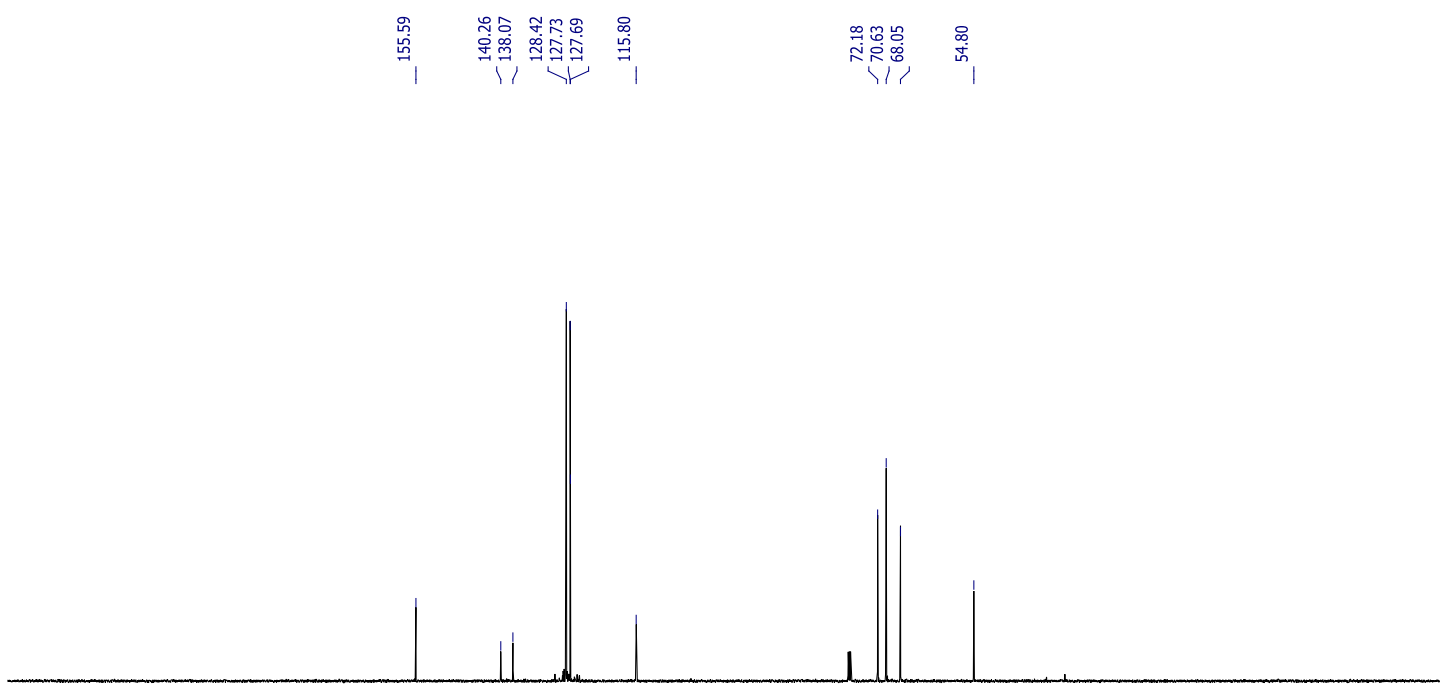

\begin{tabular}{rl|llllllllllllllllllllllllllll}
\hline 220 & 210 & 200 & 190 & 180 & 170 & 160 & 150 & 140 & 130 & 120 & 110 & 100 & 90 & 80 & 70 & 60 & 50 & 40 & 30 & 20 & 10 & 0 & -10 & -20
\end{tabular} 


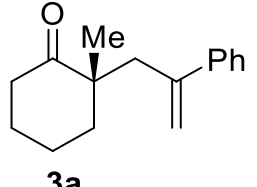

I H NMR (500 MHz, CDCl3)
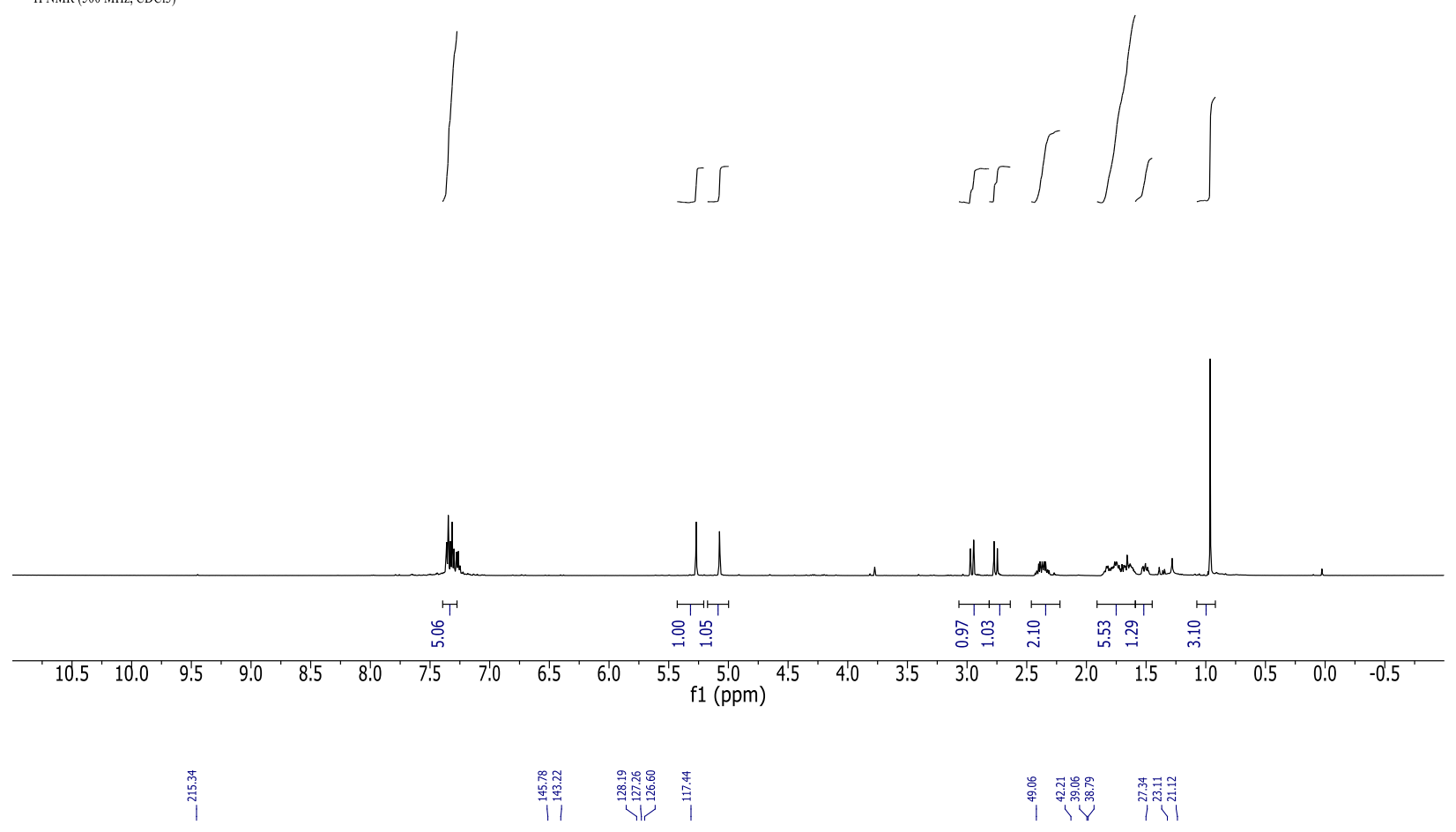

${ }^{13} \mathrm{CNMR}\left(125 \mathrm{MHz}, \mathrm{CDCl}_{3}\right)$
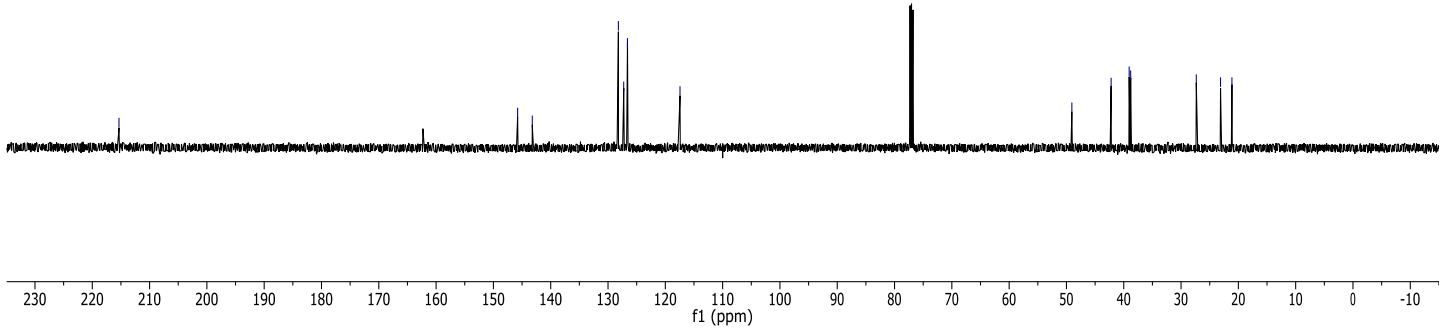


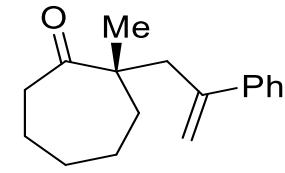

3b

ケ
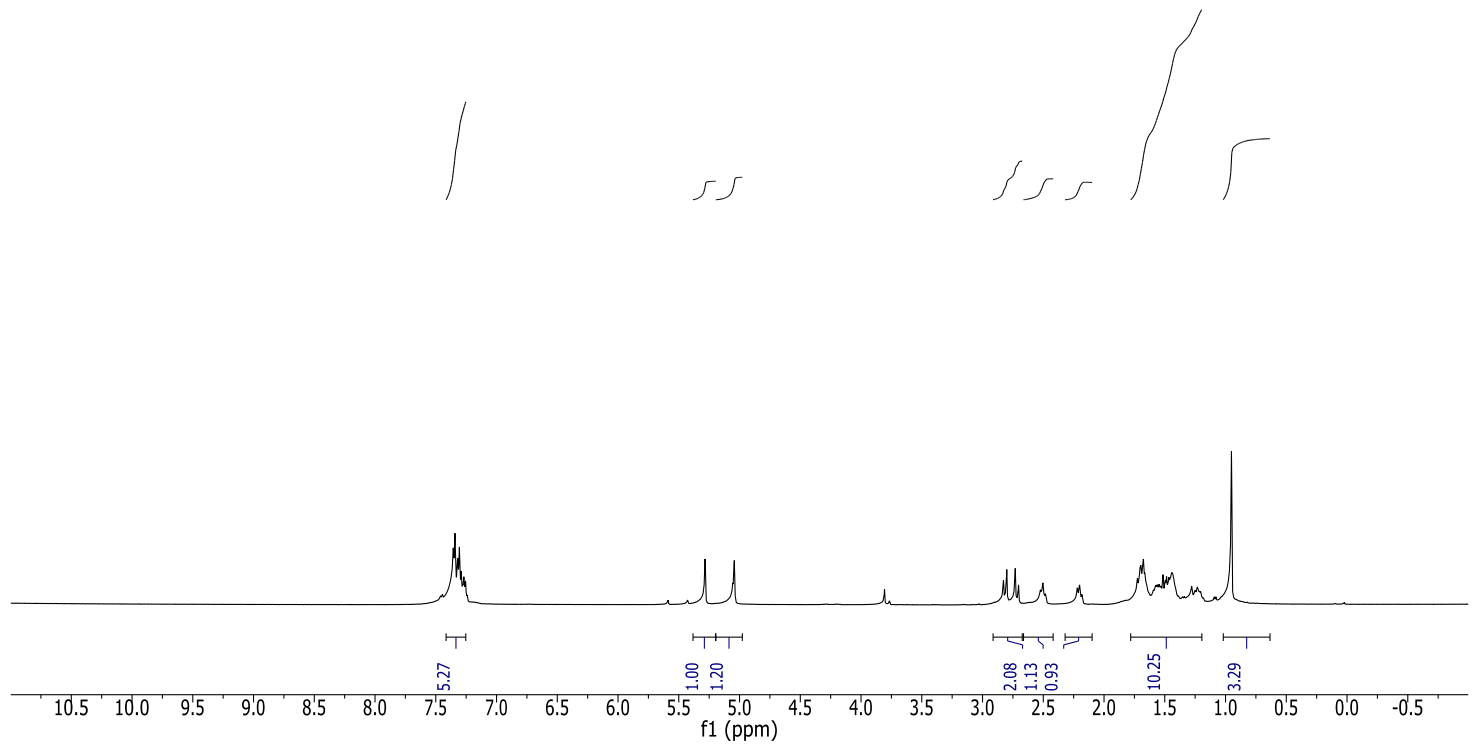

${ }^{2} \mathrm{C}$ NMR $(125 \mathrm{MHz}, \mathrm{CDCl}$;

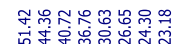

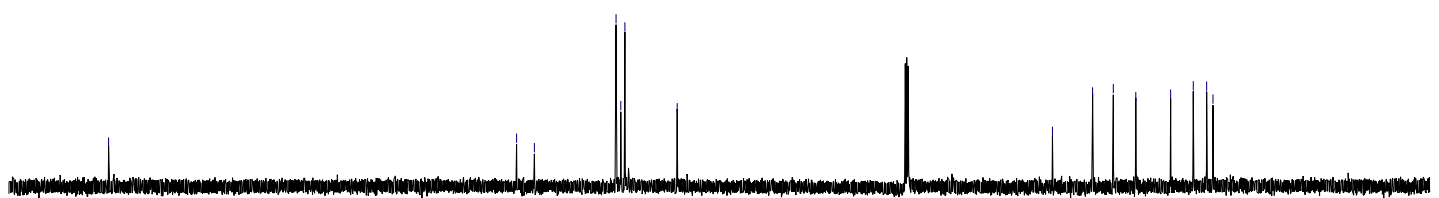

\begin{tabular}{lllllllllllllllllllllllllllllll}
\hline 230 & 220 & 210 & 200 & 190 & 180 & 170 & 160 & 150 & 140 & 130 & 120 & 110 & 100 & 90 & 80 & 70 & 60 & 50 & 40 & 30 & 20 & 10 & 0 & -10
\end{tabular} 


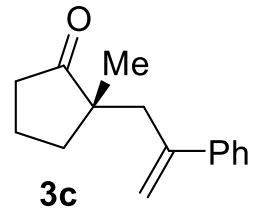

' H NMR (500 MHz, CDCl3)

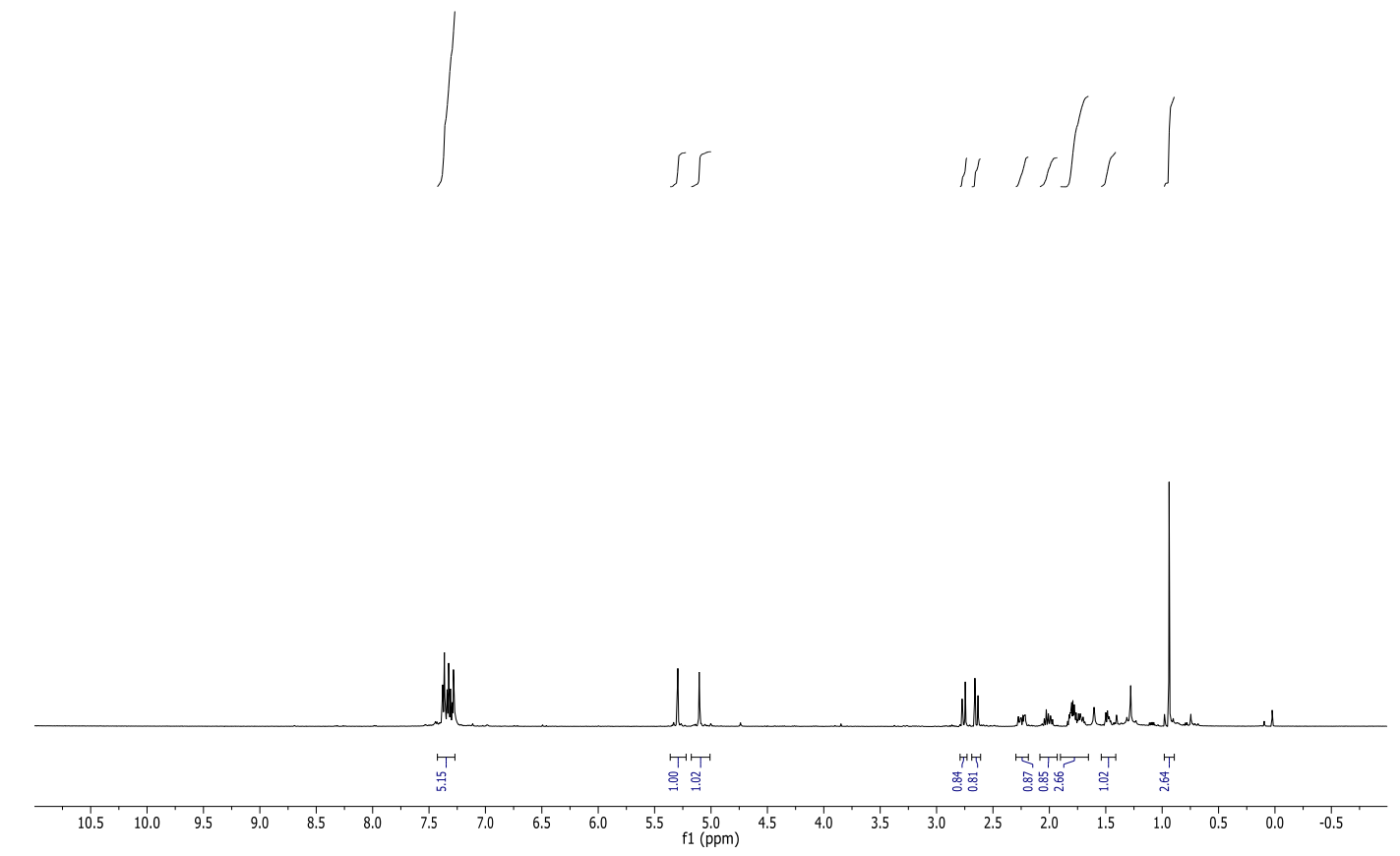

${ }^{13} \mathrm{C} \mathrm{NMR} \mathrm{(125} \mathrm{MHz,} \mathrm{CDCl3)}$

$\stackrel{\circ}{\underset{\sim}{*}}$

|

|

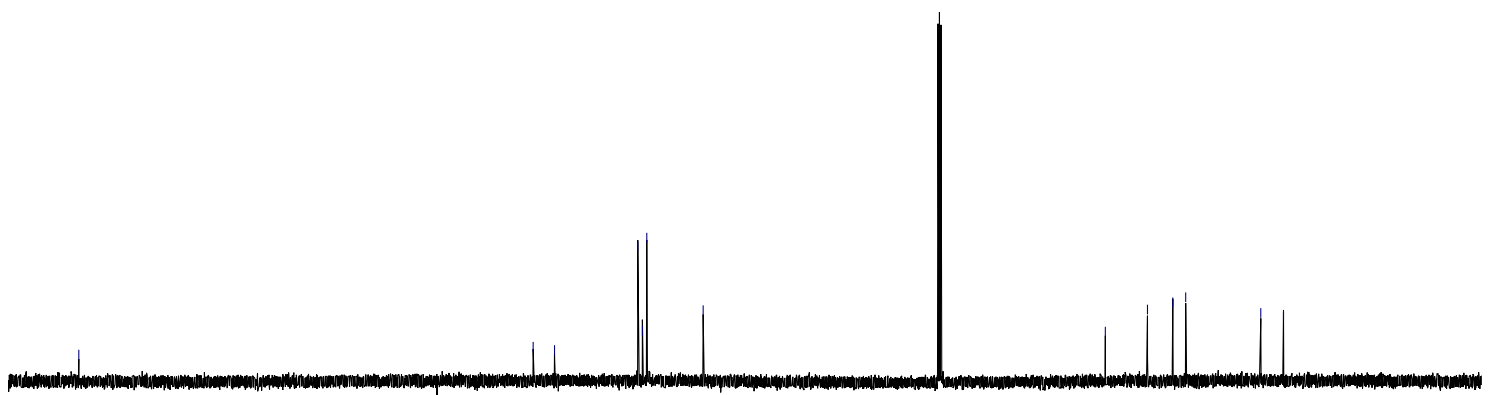

\begin{tabular}{lllllllllllllllllllllllllllll}
\hline 230 & 220 & 210 & 200 & 190 & 180 & 170 & 160 & 150 & 140 & 130 & 120 & 110 & 100 & 90 & 80 & 70 & 60 & 50 & 40 & 30 & 20 & 10 & 0 & -10
\end{tabular} 
<smiles>C=C(CC1(c2ccccc2)CCCCC1=O)c1ccccc1</smiles>

3d

' H NMR (500 MHz, CDCl3)
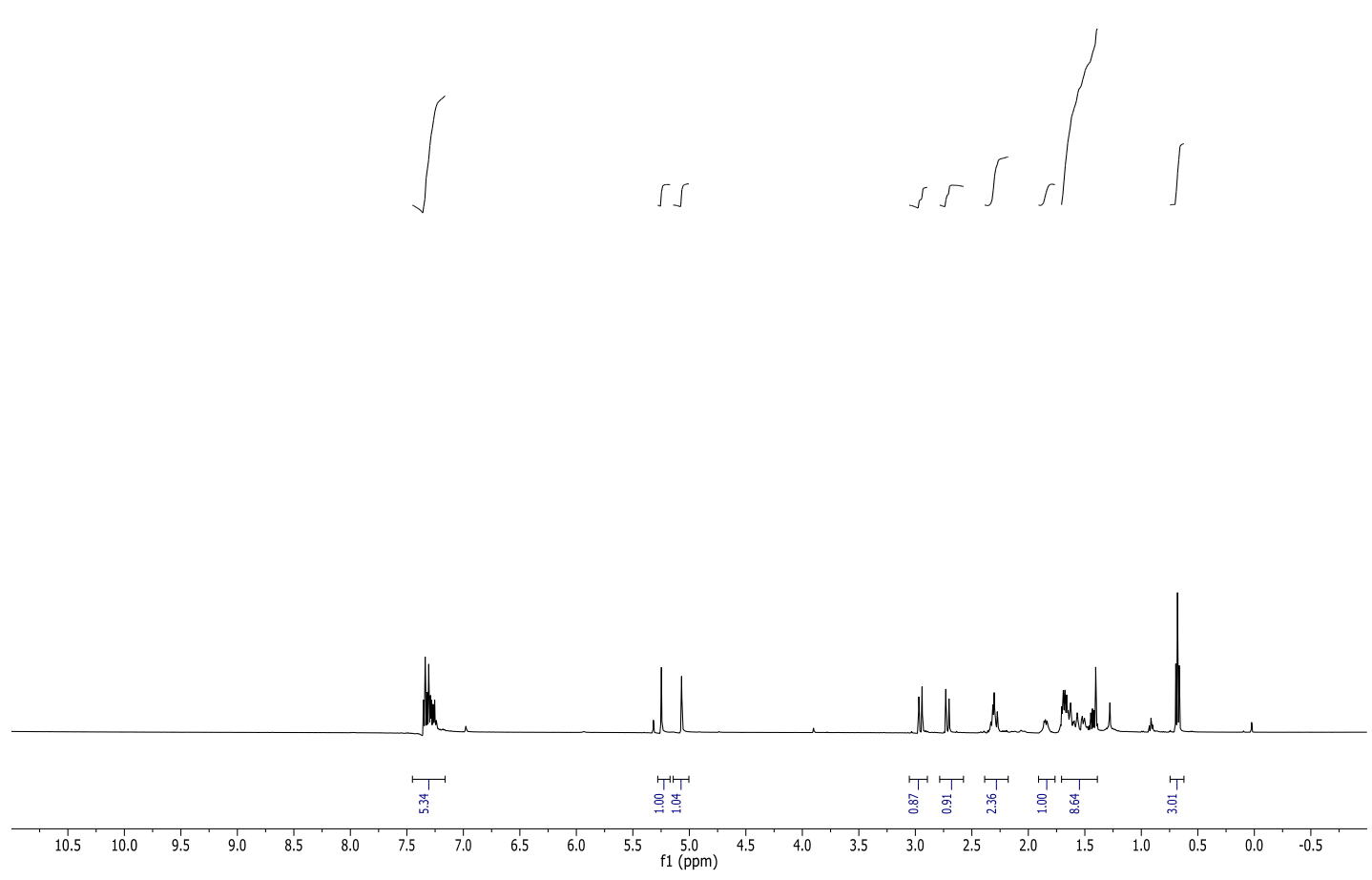

离
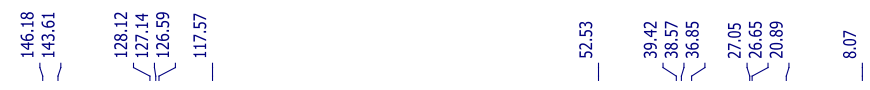

${ }^{n} \mathrm{C} \mathrm{NMR}\left(125 \mathrm{MHz}, \mathrm{CDCl}_{1}\right)$

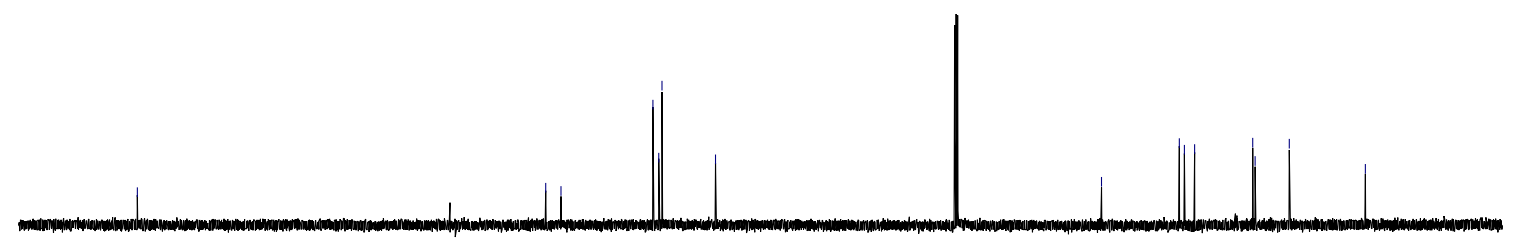

\begin{tabular}{llllllllllllllllllllllllll}
\hline 230 & 220 & 210 & 200 & 190 & 180 & 170 & 160 & 150 & 140 & 130 & 120 & 110 & 100 & 90 & 80 & 70 & 60 & 50 & 40 & 30 & 20 & 10 & 0 & -10
\end{tabular} 


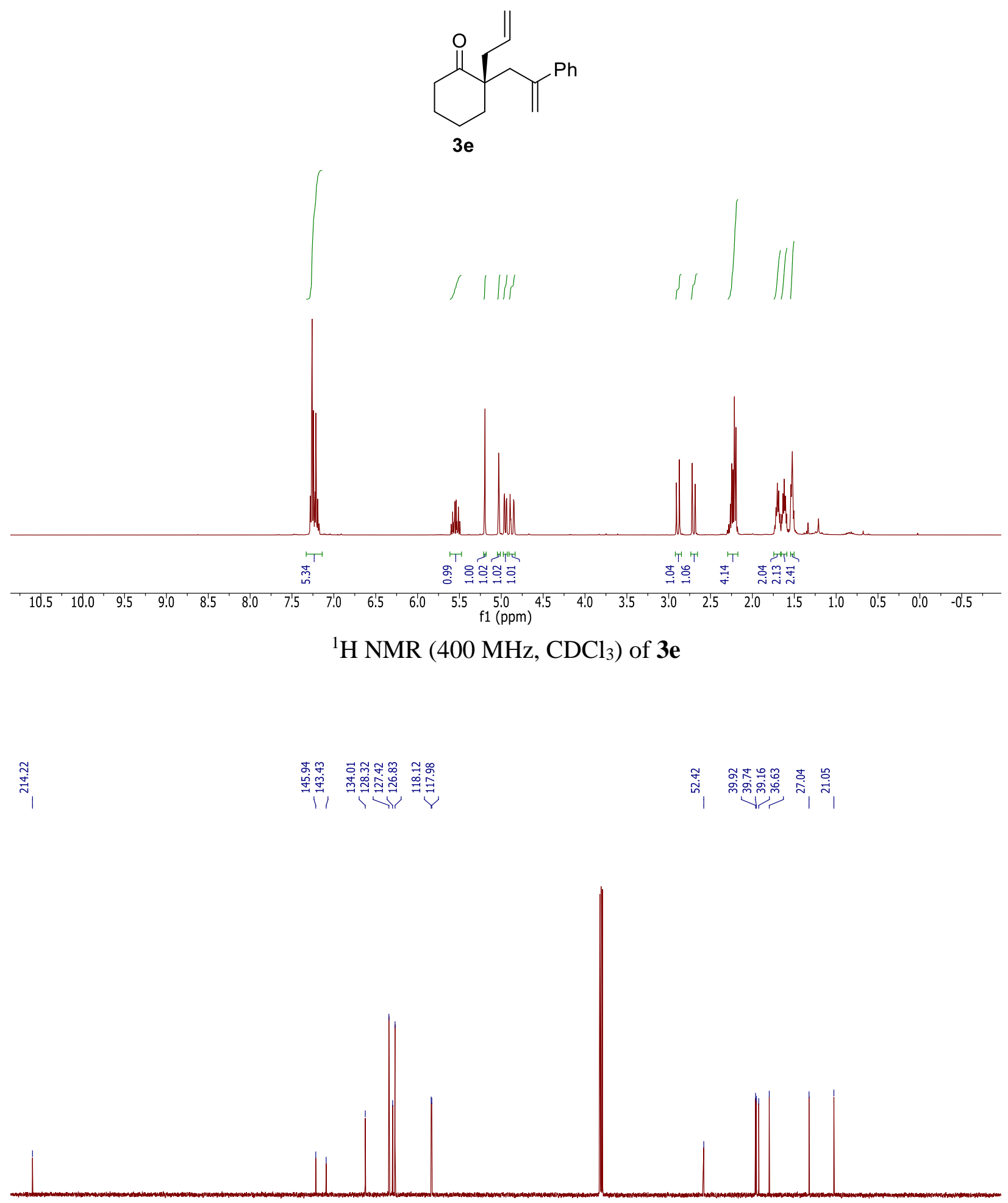

\begin{tabular}{lllllllllllllllllllllllll}
\hline 210 & 200 & 190 & 180 & 170 & 160 & 150 & 140 & 130 & 120 & 110 & 100 & 90 & 80 & 70 & 60 & 50 & 40 & 30 & 20 & 10 & 0 & -10 & 10
\end{tabular} ${ }^{13} \mathrm{C} \mathrm{NMR}\left(100 \mathrm{MHz}, \mathrm{CDCl}_{3}\right)$ of $\mathbf{3 e}$ 

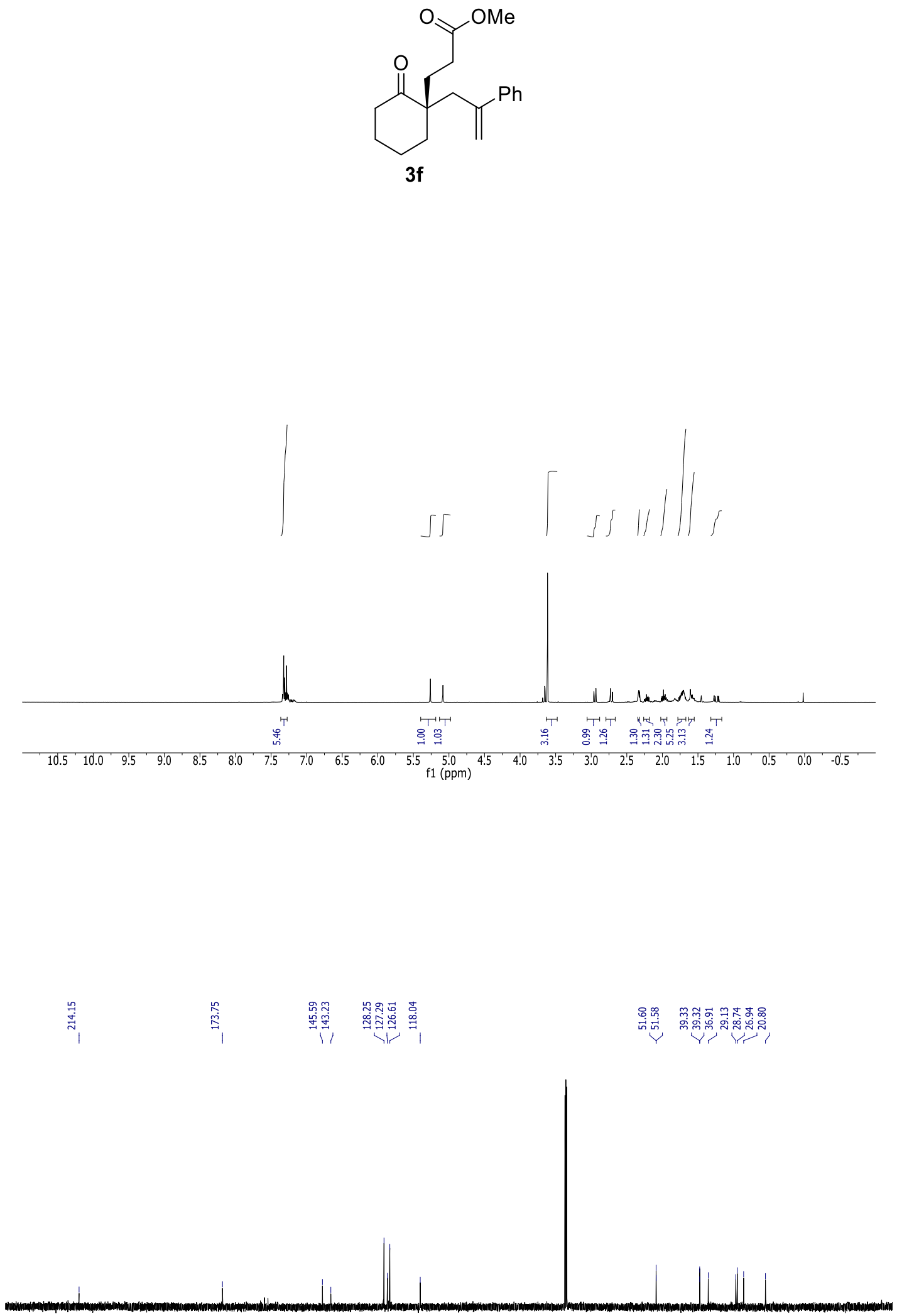

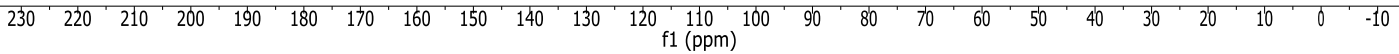


$\underbrace{\mathrm{Bn}}_{1}$

$3 g$

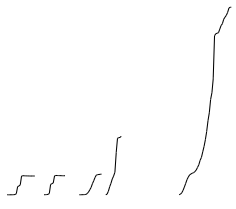
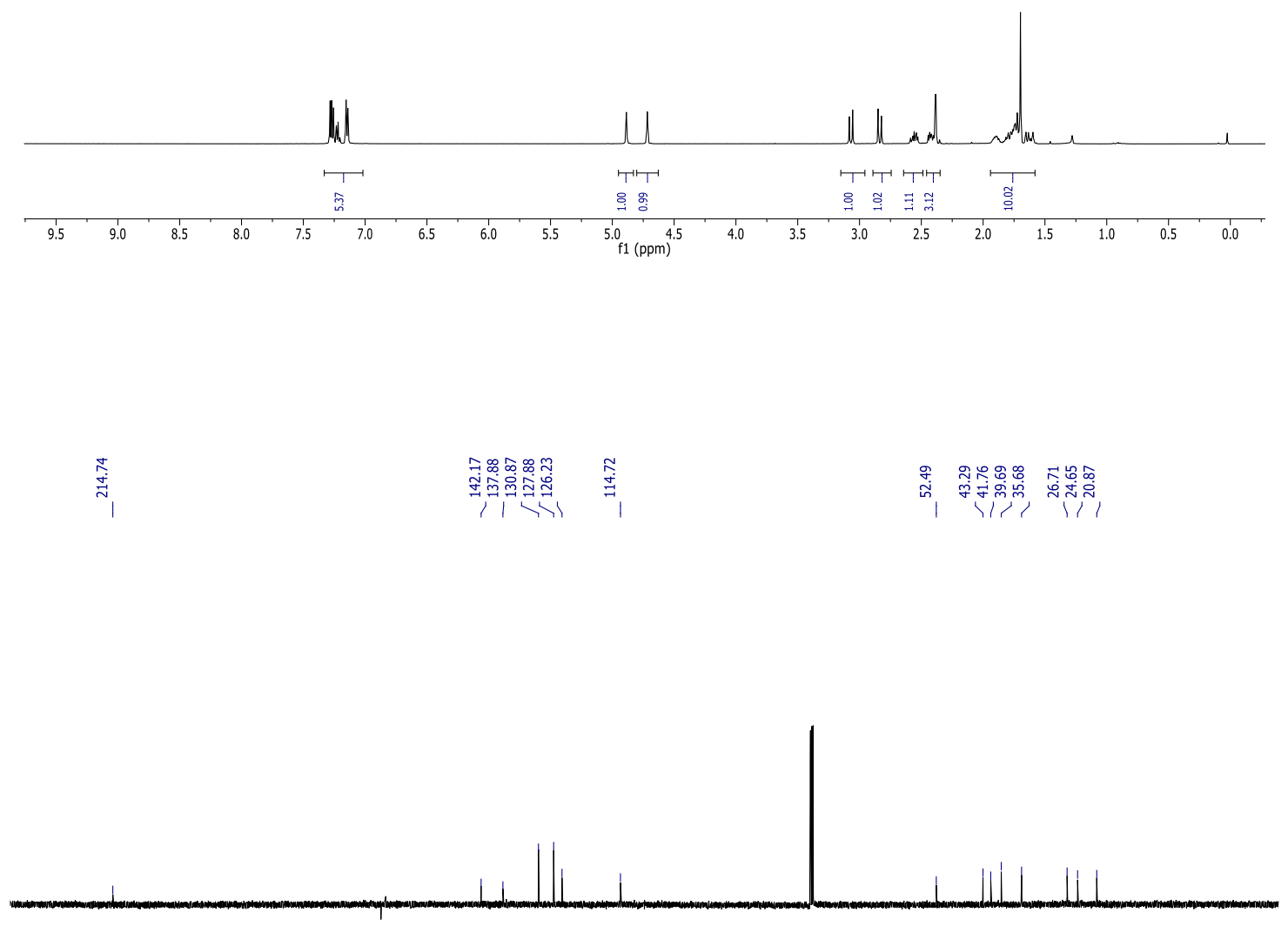

\begin{tabular}{l|l|l|l|l|l|l|l|l|l|l|l|lllllllllllllll}
\hline 230 & 220 & 210 & 200 & 190 & 180 & 170 & 160 & 150 & 140 & 130 & 120 & 110 & 100 & 90 & 80 & 70 & 60 & 50 & 40 & 30 & 20 & 10 & 0 & -10
\end{tabular} 


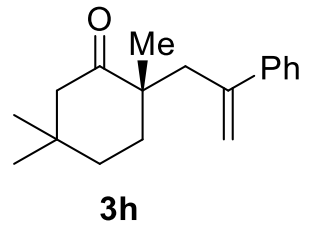

' H NMR (500 MHz, CDCl3)
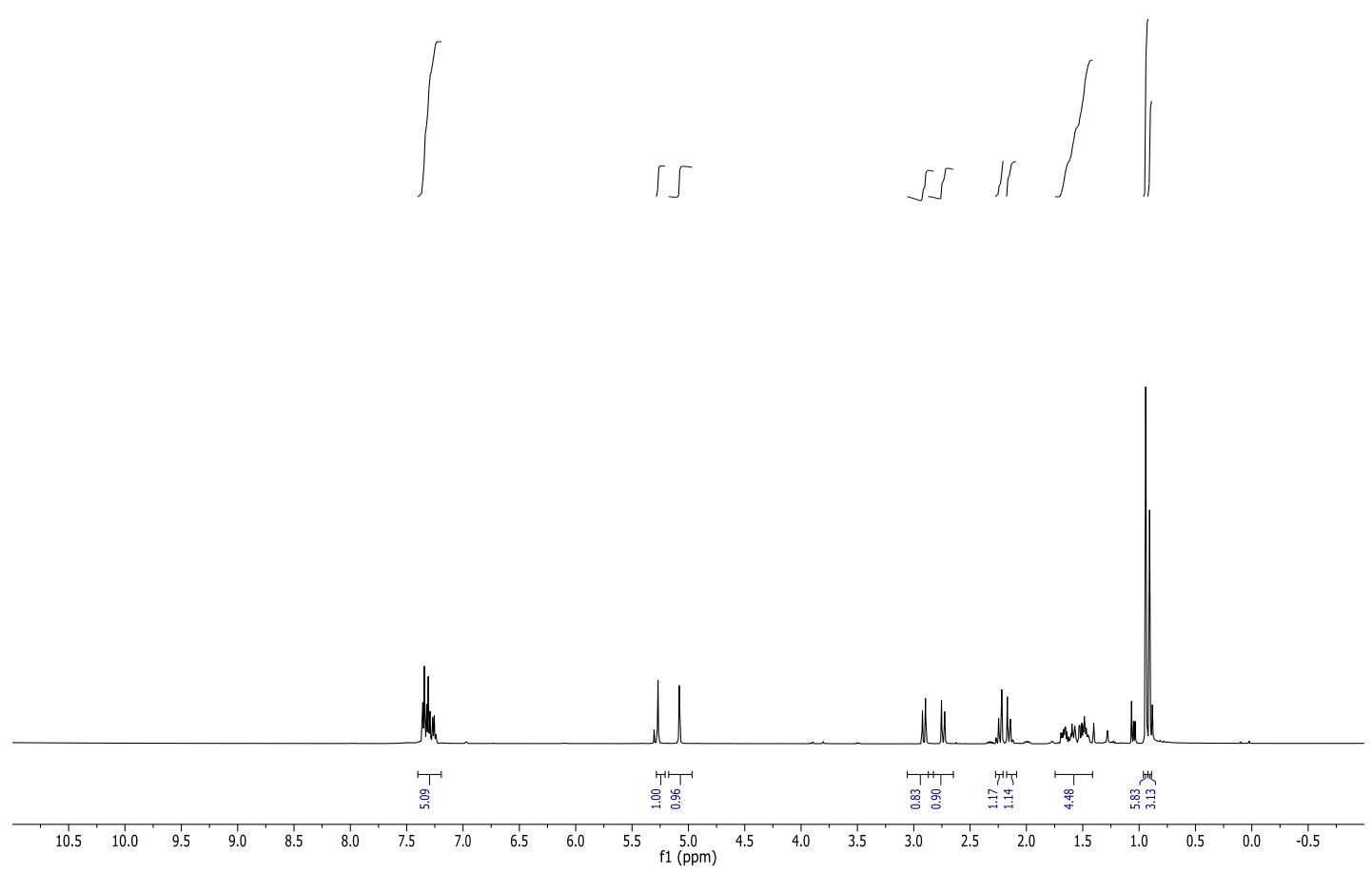

${ }^{\mathrm{B}} \mathrm{CNMR}\left(125 \mathrm{MHz}, \mathrm{CDCl}_{3}\right)$

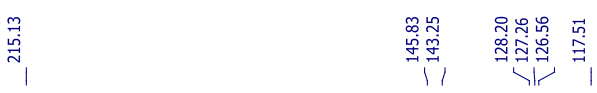

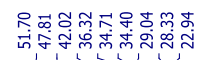

$11<1$

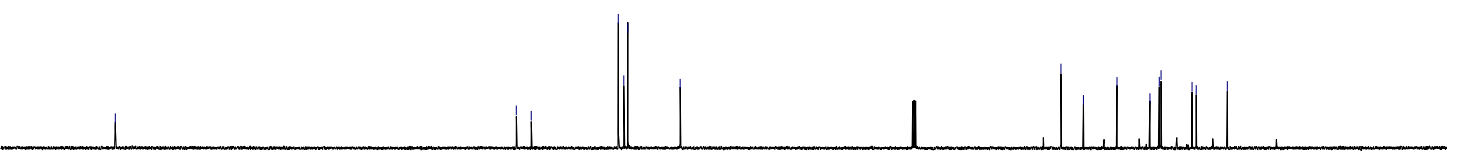

\begin{tabular}{lllllllllllllllllllllllllllllllll}
\hline 230 & 220 & 210 & 200 & 190 & 180 & 170 & 160 & 150 & 140 & 130 & 120 & 110 & 100 & 90 & 80 & 70 & 60 & 50 & 40 & 30 & 20 & 10 & 0 & -10
\end{tabular} 


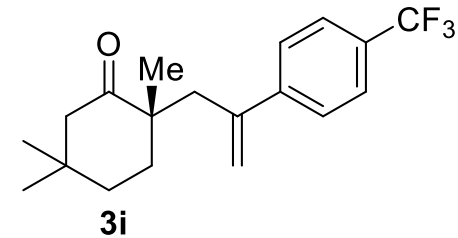

${ }^{\prime} \mathrm{H}$ NMR (500 MHz, CDCl3)

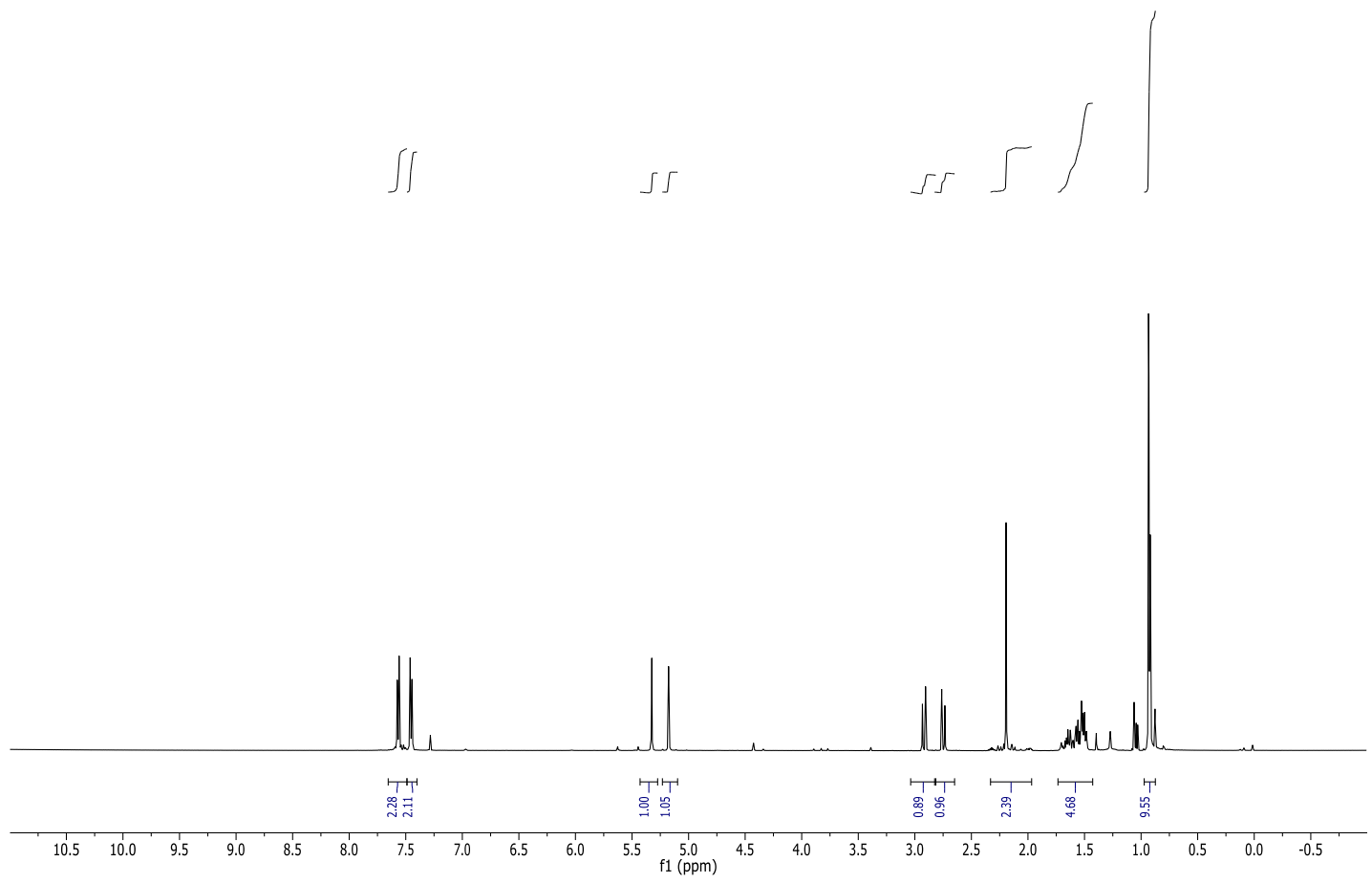

${ }^{15} \mathrm{CNMR}\left(125 \mathrm{MHz}, \mathrm{CDCl}_{1}\right)$

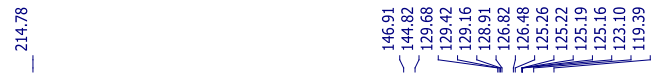

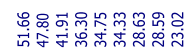

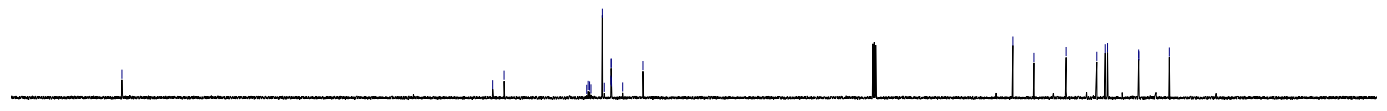

\begin{tabular}{lllllllllllllllllllllllllll}
\hline 230 & 220 & 210 & 200 & 190 & 180 & 170 & 160 & 150 & 140 & 130 & 120 & 110 & 100 & 90 & 80 & 70 & 60 & 50 & 40 & 30 & 20 & 10 & 0 & -10
\end{tabular} 


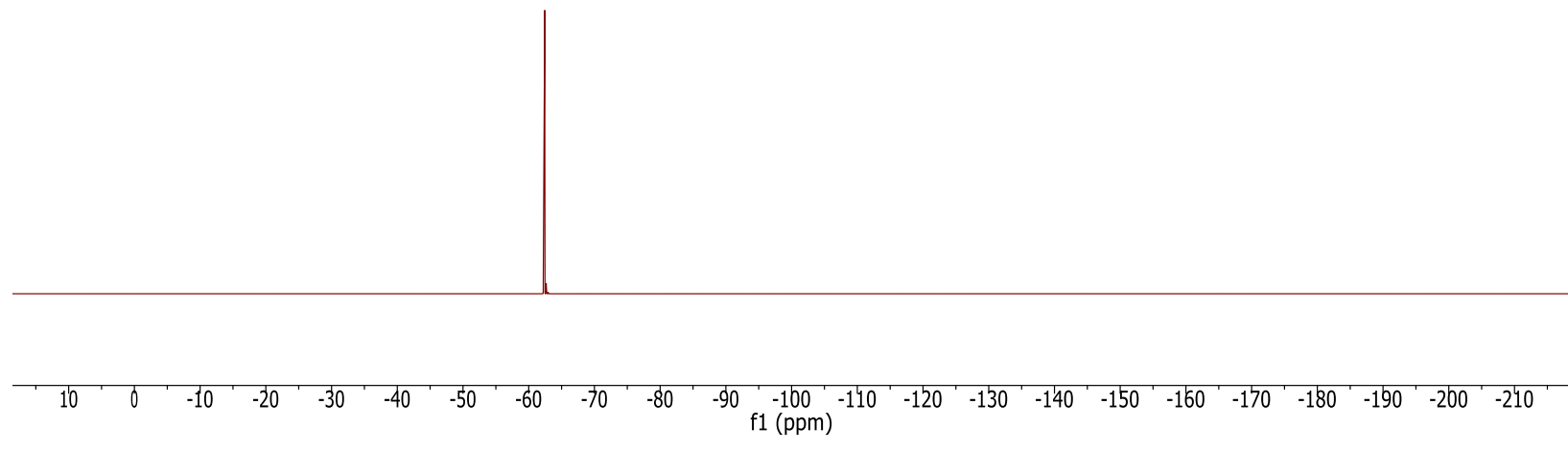

${ }^{19} \mathrm{~F} \mathrm{NMR}\left(400 \mathrm{MHz}, \mathrm{CDCl}_{3}\right)$ of $\mathbf{3 i}$ 
<smiles>C=C(CC1(C)CCC(C)(C)CC1=O)c1ccc(F)cc1</smiles>

${ }^{1} \mathrm{H} \mathrm{NMR}(500 \mathrm{MHz}, \mathrm{CDCl} 3)$

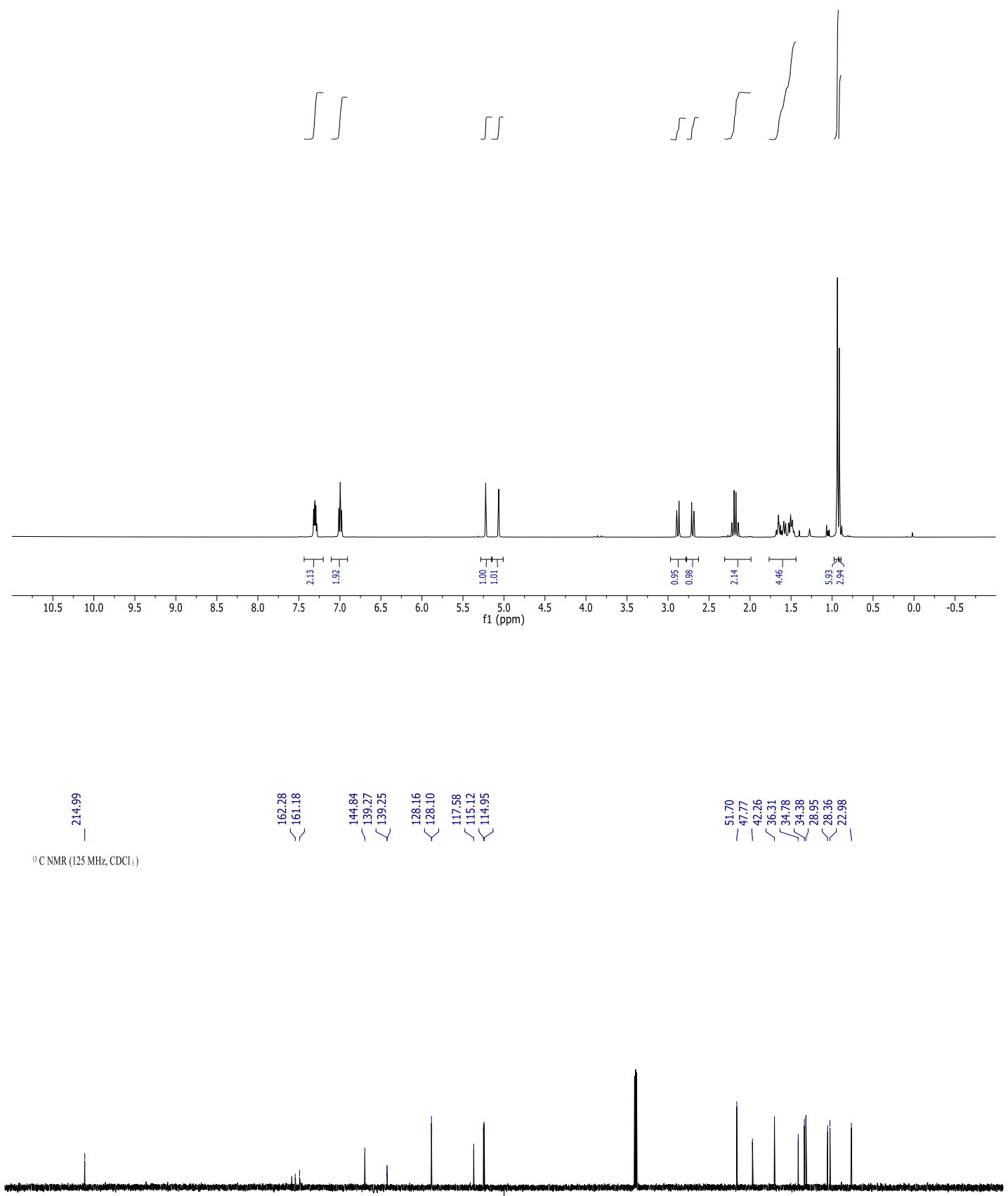

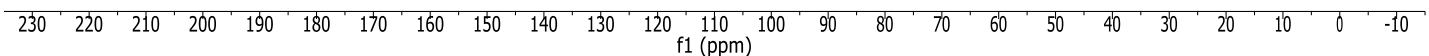




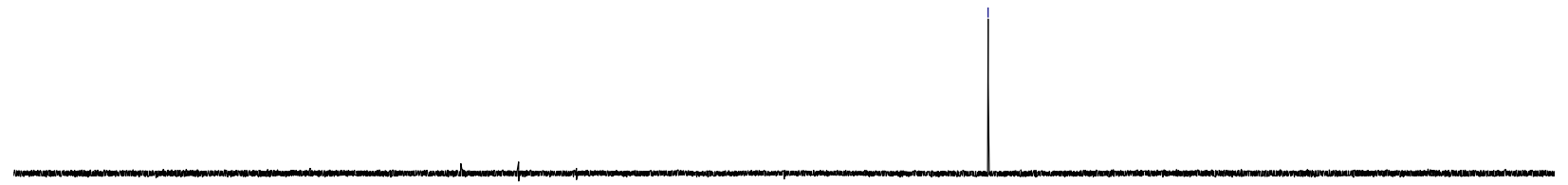

\begin{tabular}{lllllllllllllllllllllllll}
\hline 0 & 20 & 10 & 0 & -10 & -20 & -30 & -40 & -50 & -60 & -70 & -80 & -90 & -100 & -110 & -120 & -130 & -140 & -150 & -160 & -170 & -180 & -190 & -2
\end{tabular} 


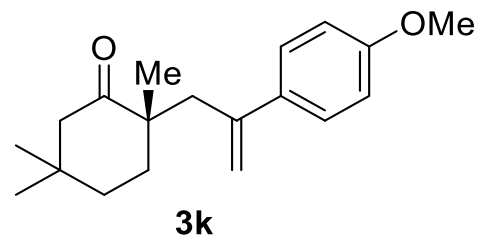

${ }^{\prime} \mathrm{H} \mathrm{NMR}(500 \mathrm{MHz}, \mathrm{CDCl} 3)$

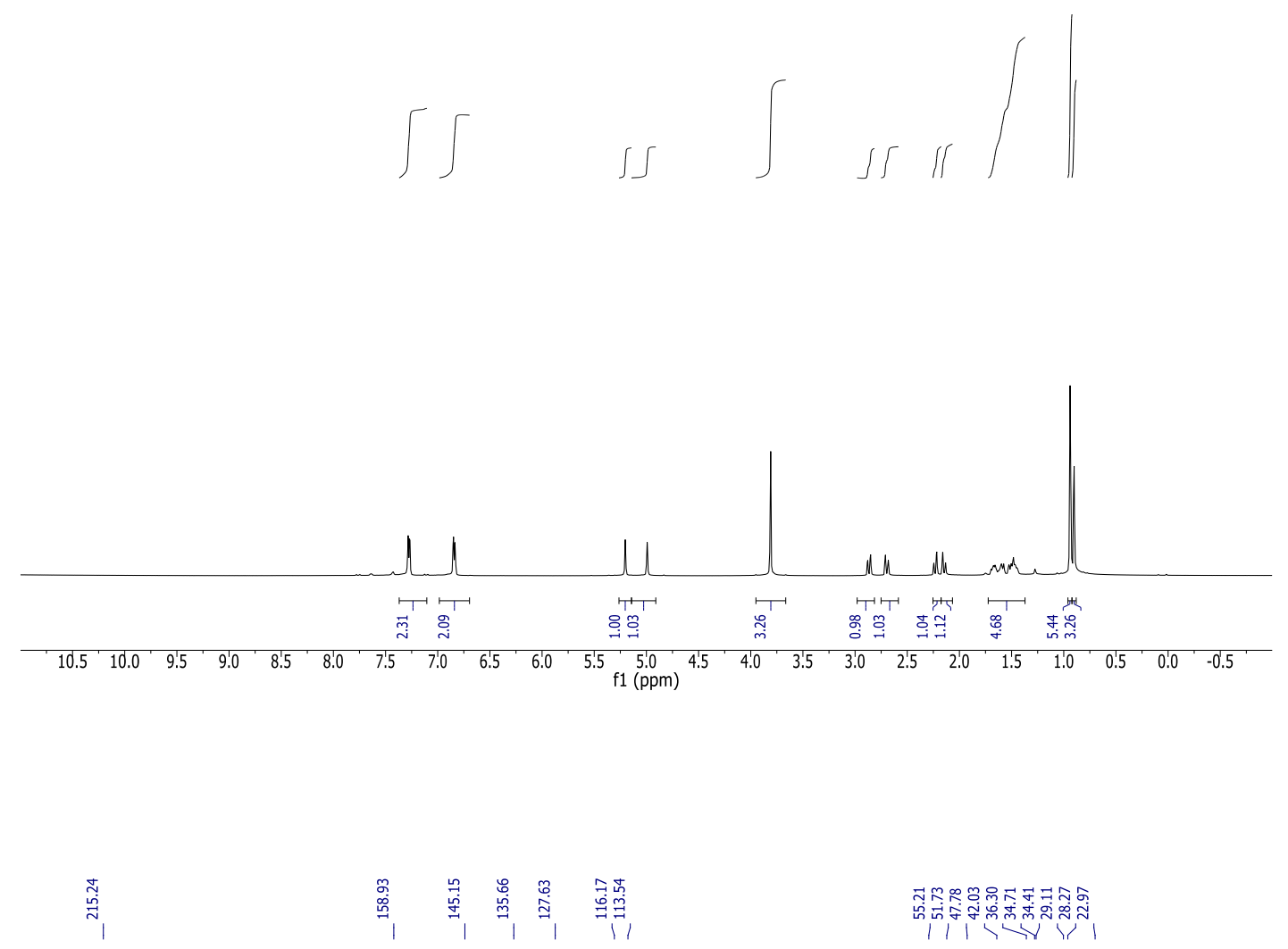

${ }^{13} \mathrm{CNMR}\left(125 \mathrm{MHz}, \mathrm{CDCl}_{3}\right)$

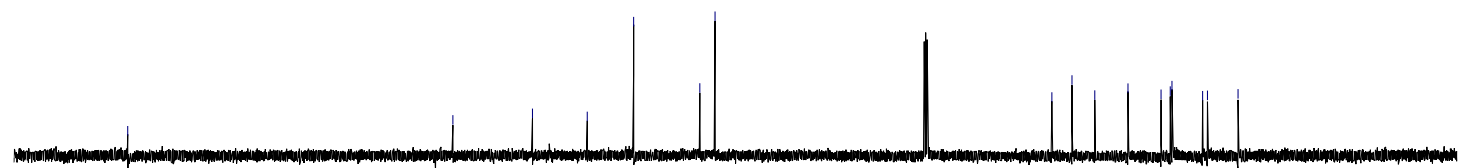

\begin{tabular}{llllllllllllllllllllllllllllll}
\hline 230 & 220 & 210 & 200 & 190 & 180 & 170 & 160 & 150 & 140 & 130 & 120 & 110 & 100 & 90 & 80 & 70 & 60 & 50 & 40 & 30 & 20 & 10 & 0 & -10
\end{tabular} 
<smiles>C=C(C[C@]1(C)CCC(C)(C)CC1=O)c1cccc(C)c1</smiles>

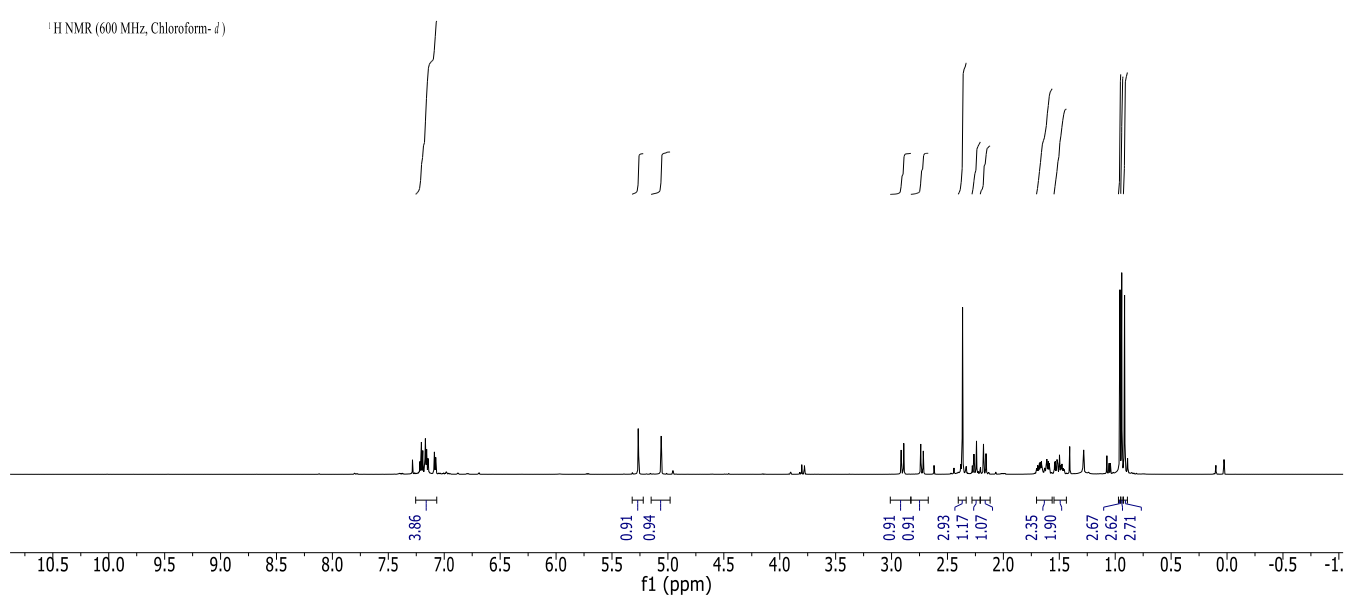

${ }^{13} \mathrm{CNMR}\left(150 \mathrm{MHz}, \mathrm{CDCl}_{3}\right)$

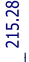

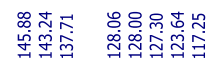

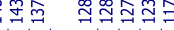

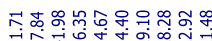

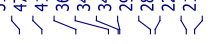

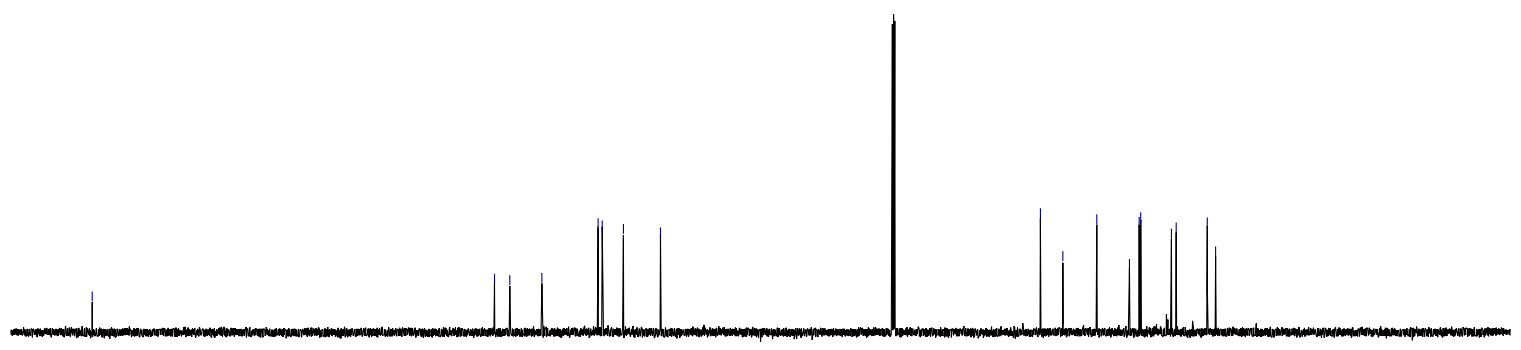

$\begin{array}{lllllllllllllllllllllllllllllllll}220 & 210 & 200 & 190 & 180 & 170 & 160 & 150 & 140 & 130 & 120 & 110 & 100 & 90 & 80 & 70 & 60 & 50 & 40 & 30 & 20 & 10 & 0 & -10 & -20\end{array}$ 
<smiles>C=C(CC1(C)CCC(C)(C)CC1=O)c1ccco1</smiles>

$3 \mathrm{~m}$

${ }^{1}$ H NMR (600 MHz, Chloroform- $d$ )
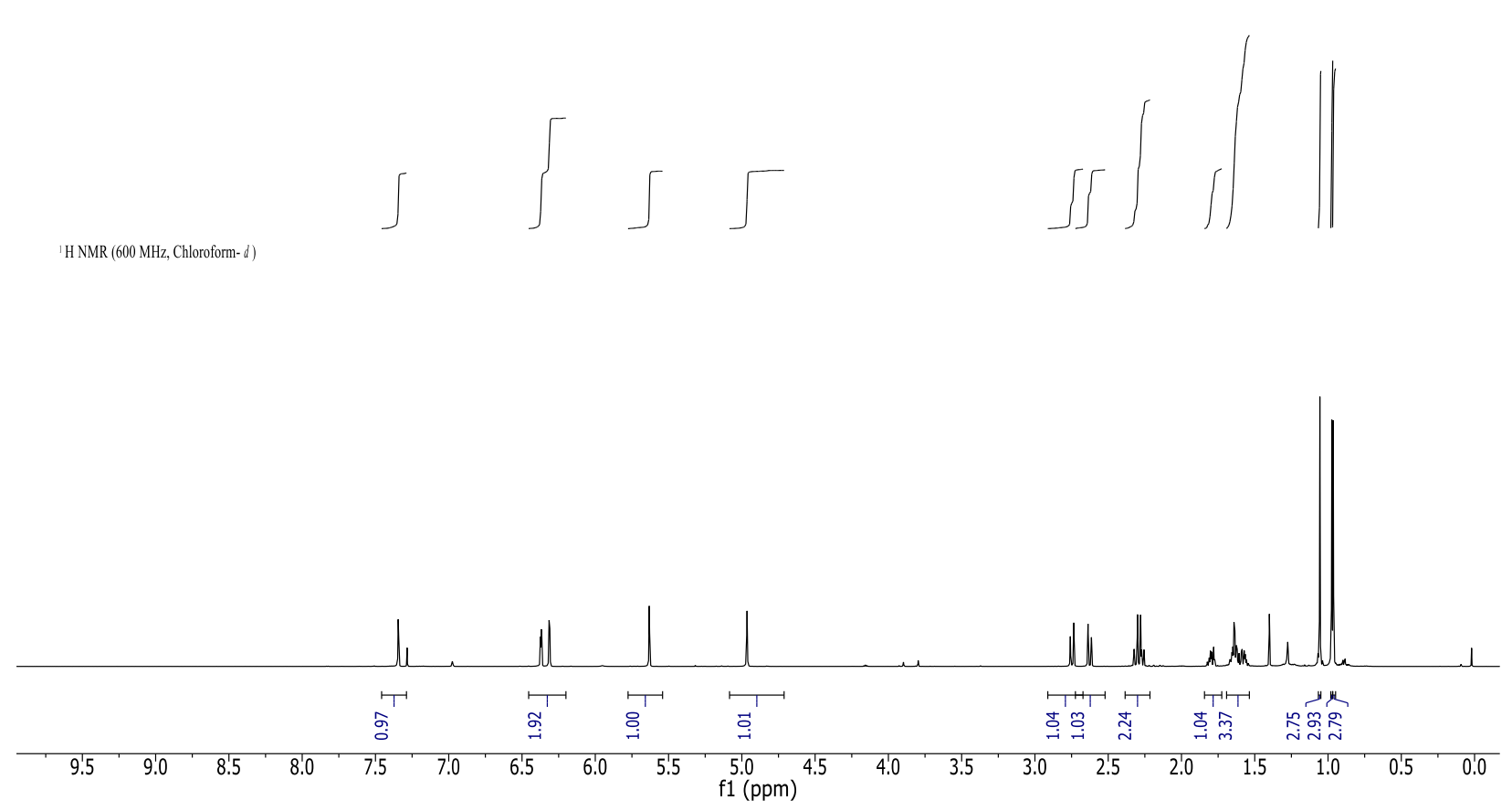

${ }^{13} \mathrm{CNMR}\left(150 \mathrm{MHz}, \mathrm{CDCl}_{3}\right)$
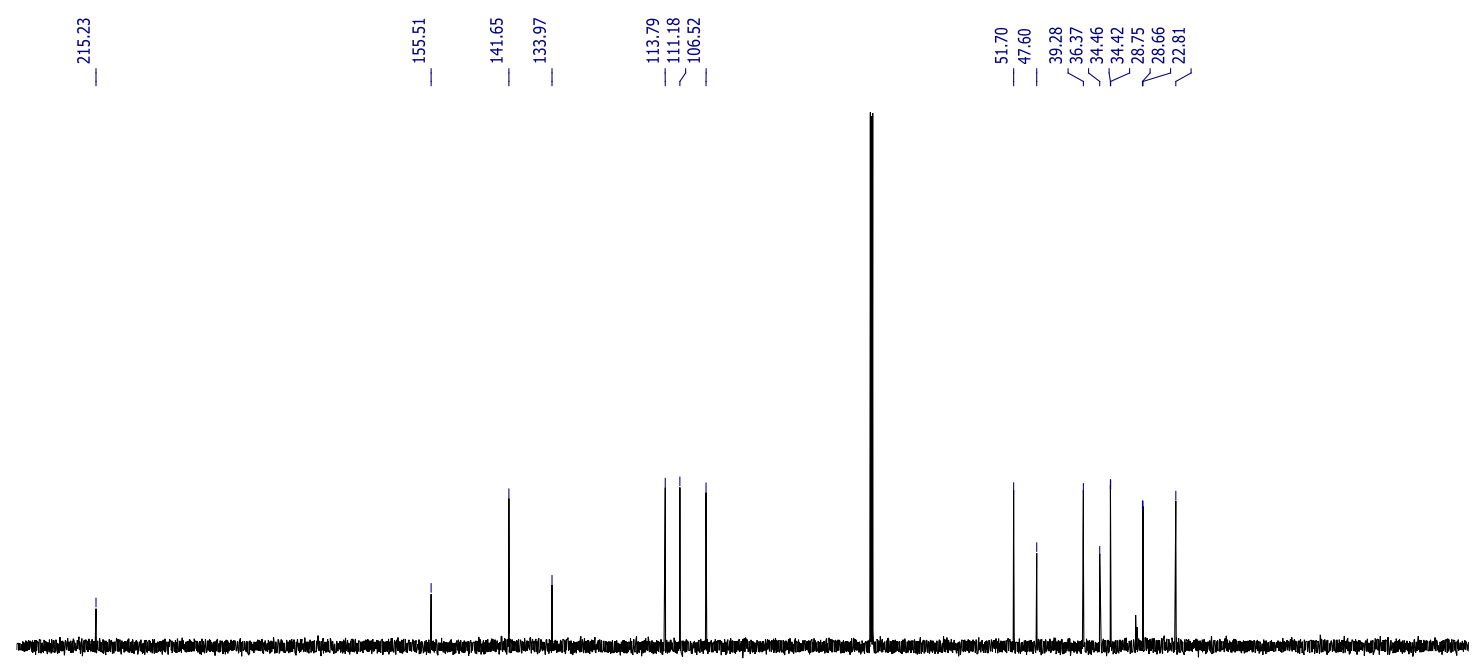

$\begin{array}{llllllllllllllllllllllllllllllll}220 & 210 & 200 & 190 & 180 & 170 & 160 & 150 & 140 & 130 & 120 & 110 & 100 & 90 & 80 & 70 & 60 & 50 & 40 & 30 & 20 & 10 & 0 & -10 & -20 & \end{array}$ 


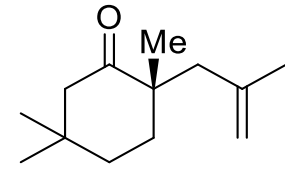

30

IH NMR (300 MHz, Chloroform- $d) 8$ $\iint||||$

wh th

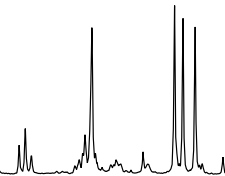

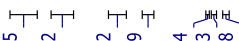

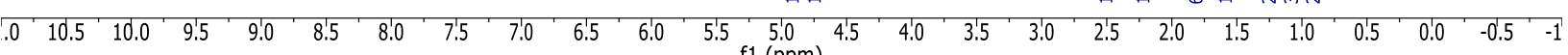

${ }^{3} \mathrm{CNMR}\left(125 \mathrm{MHz}, \mathrm{CDCl}_{3}\right)$

至|

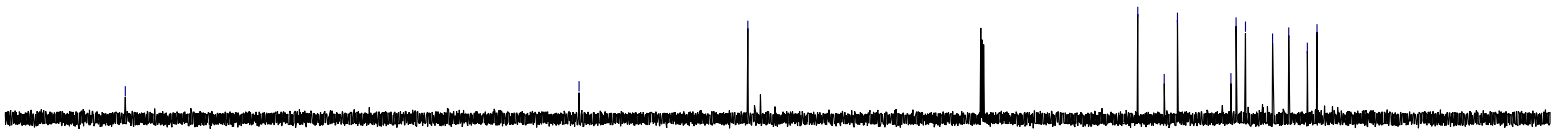

$\begin{array}{llllllllllllllllllllllllllll}230 & 220 & 210 & 200 & 190 & 180 & 170 & 160 & 150 & 140 & 130 & 120 & 110 & 100 & 90 & 80 & 70 & 60 & 50 & 40 & 30 & 20 & 10 & 0 & -10\end{array}$ 
<smiles>C=C(COCc1ccccc1)CC1(C)CCC(C)(C)CC1=O</smiles>
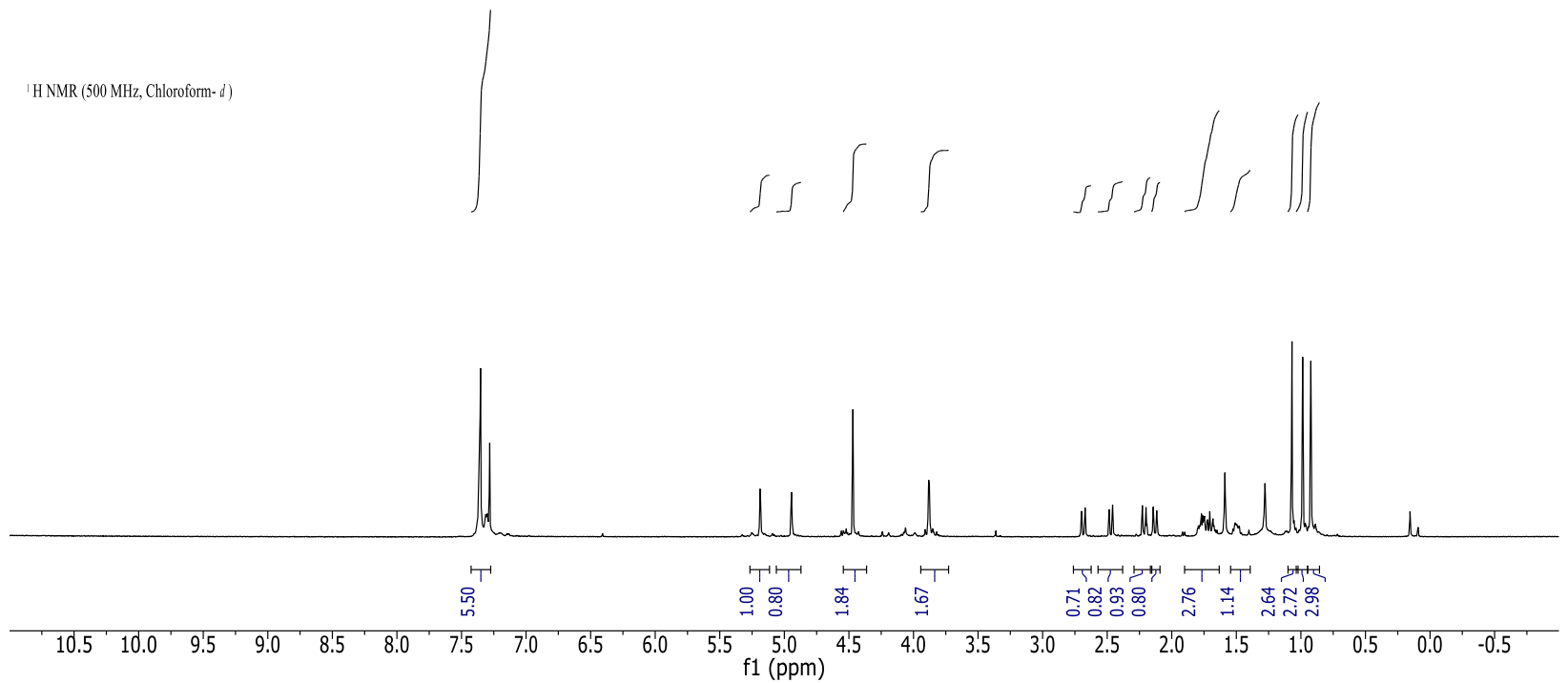

${ }^{18} \mathrm{C} \mathrm{NMR}\left(125 \mathrm{MHz}, \mathrm{CDCl}_{3}\right)$
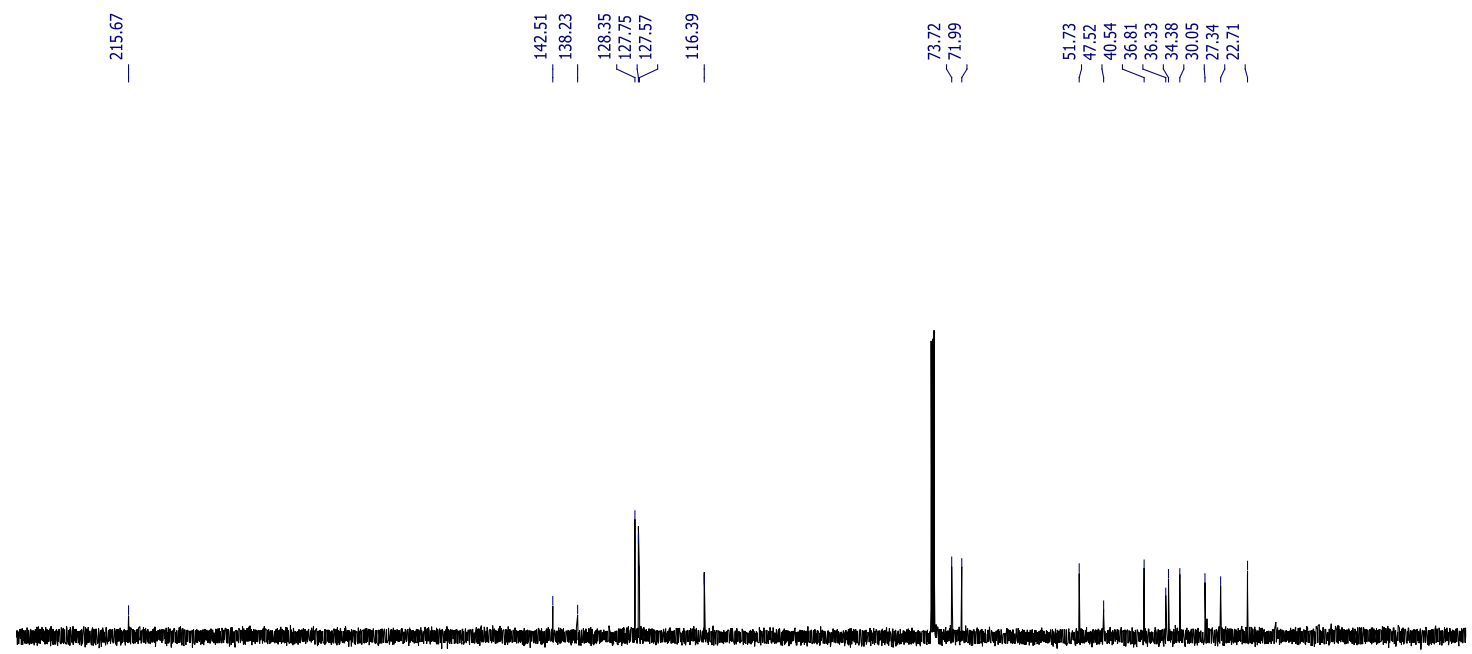

\begin{tabular}{llllllllllllllllllllllllllll}
\hline 230 & 220 & 210 & 200 & 190 & 180 & 170 & 160 & 150 & 140 & 130 & 120 & 110 & 100 & 90 & 80 & 70 & 60 & 50 & 40 & 30 & 20 & 10 & 0 & -10
\end{tabular} 


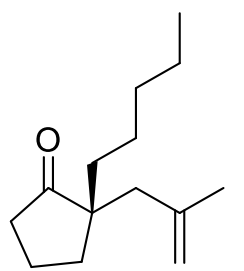

$3 q$

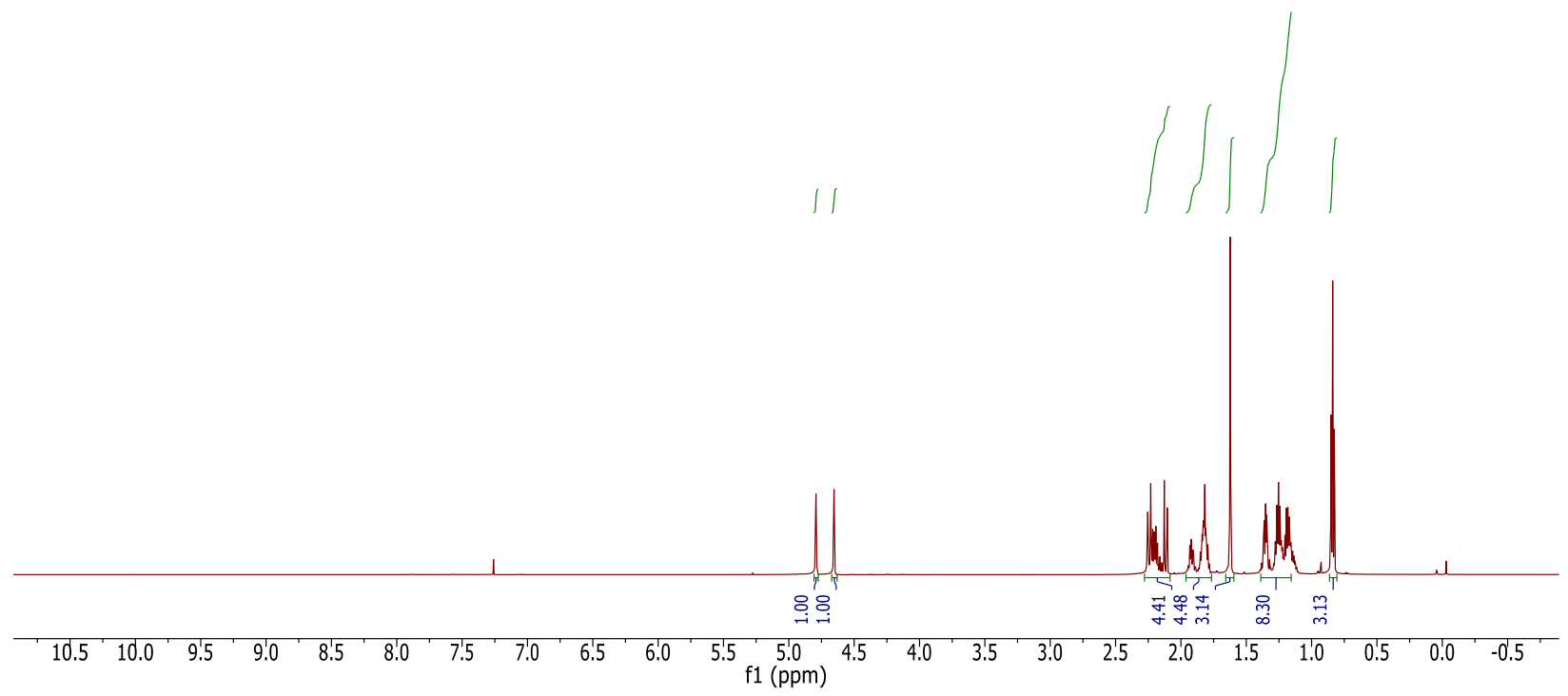

${ }^{13} \mathrm{CNMR}\left(125 \mathrm{MHz}, \mathrm{CDCl}_{3}\right)$

लำ

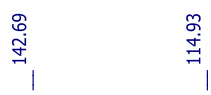

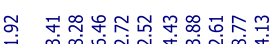

ก

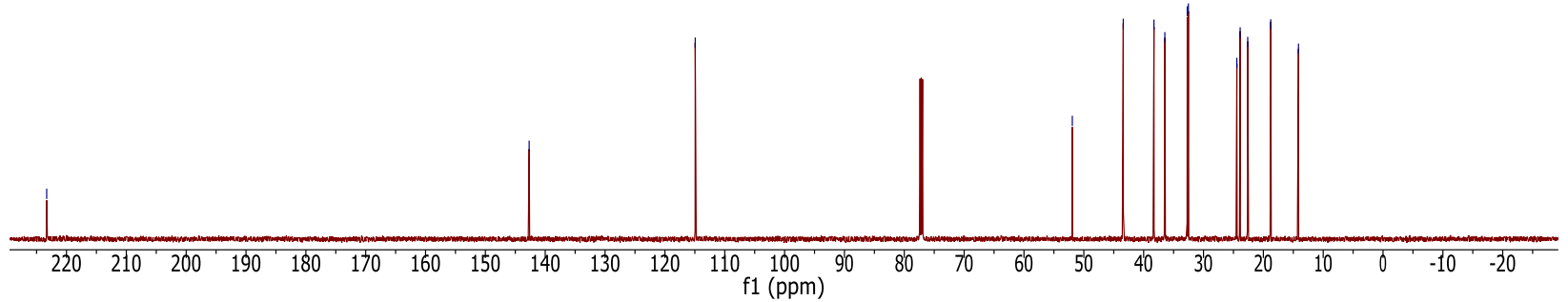




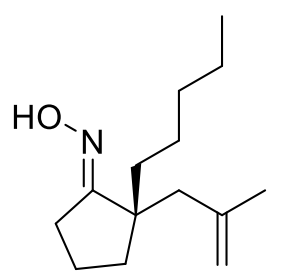

$3 q 1$
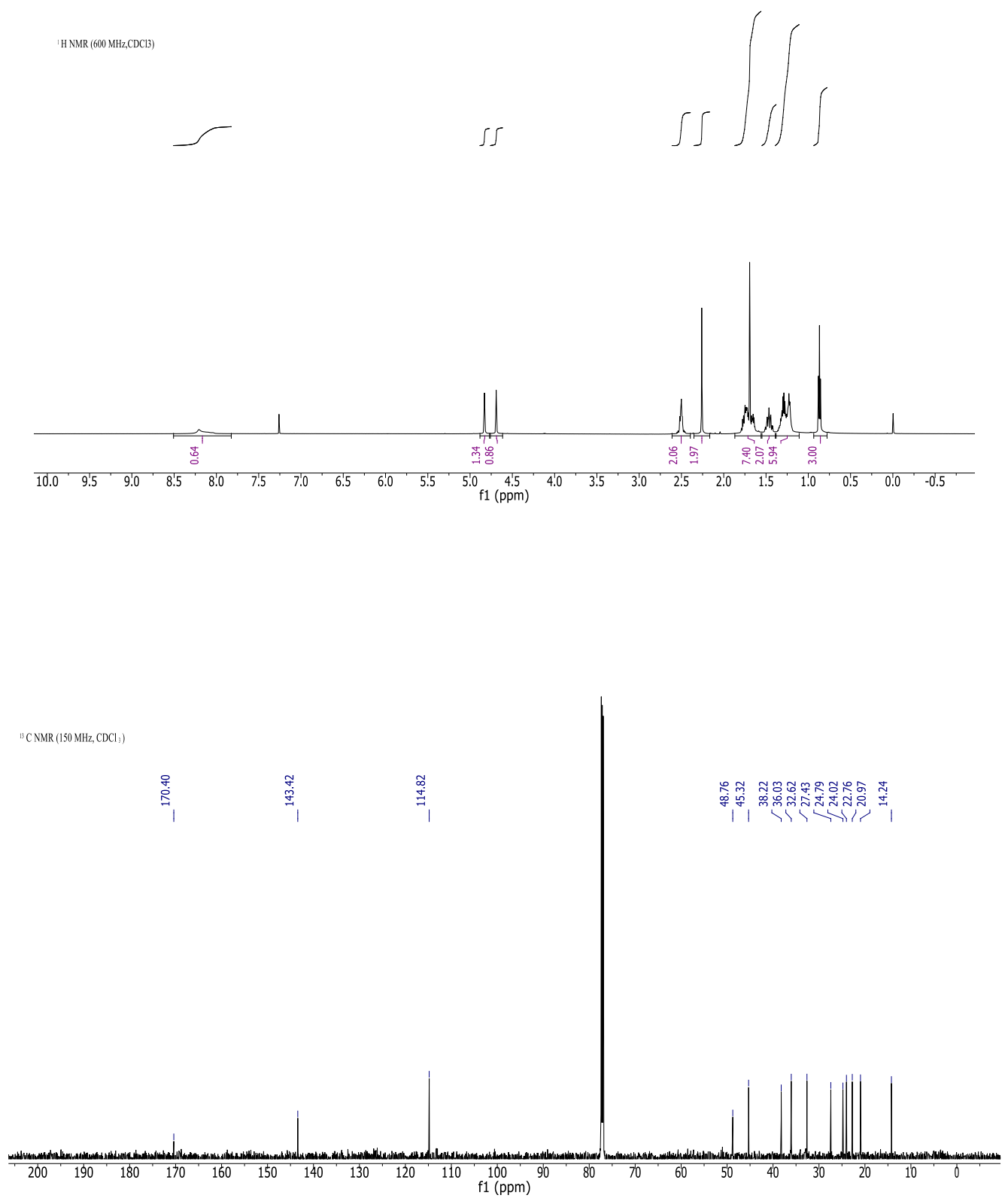

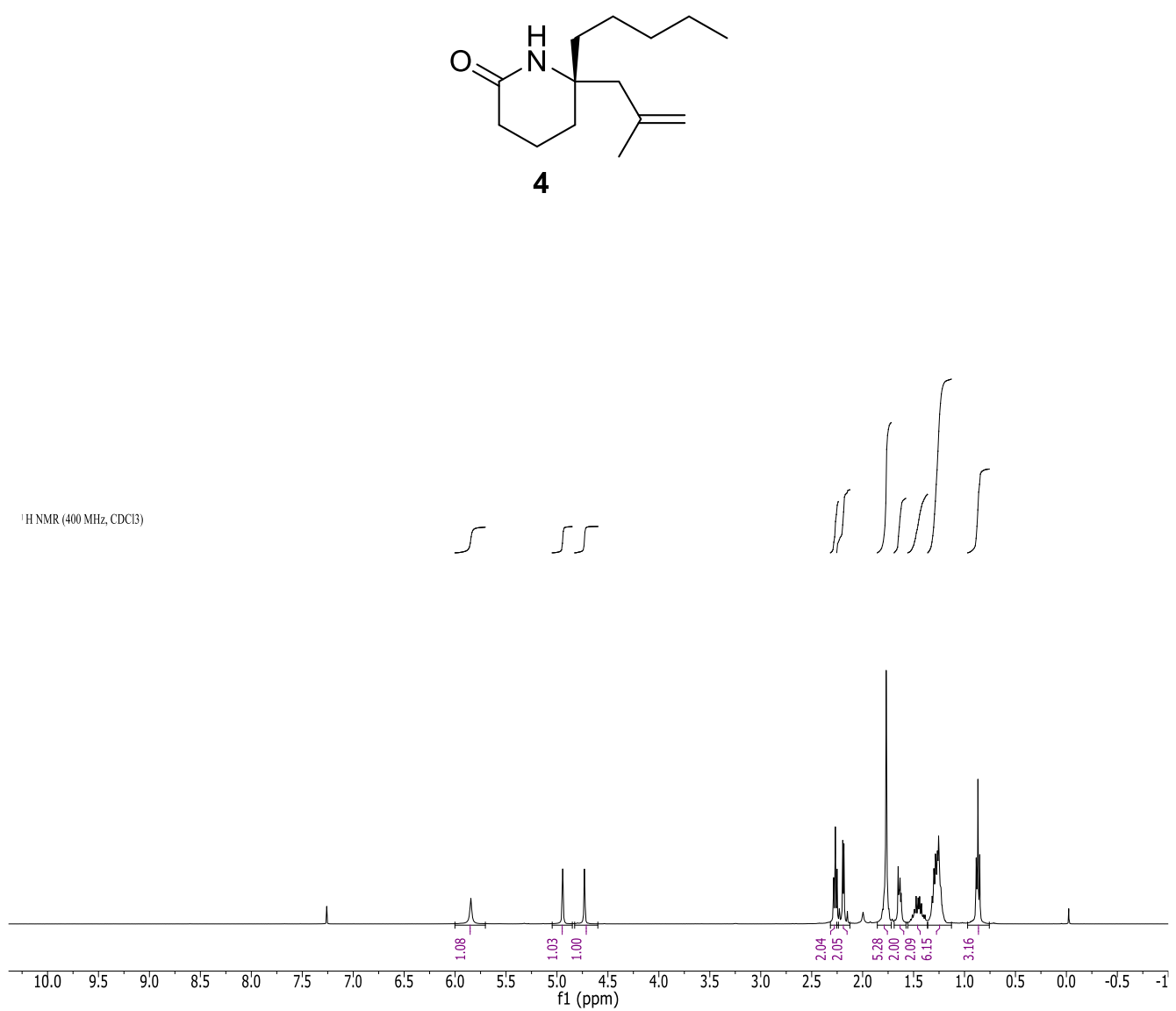

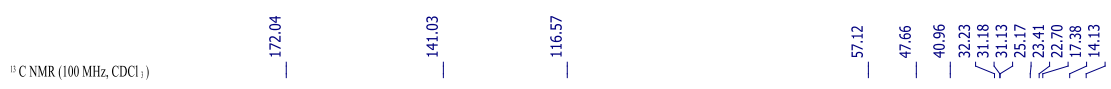

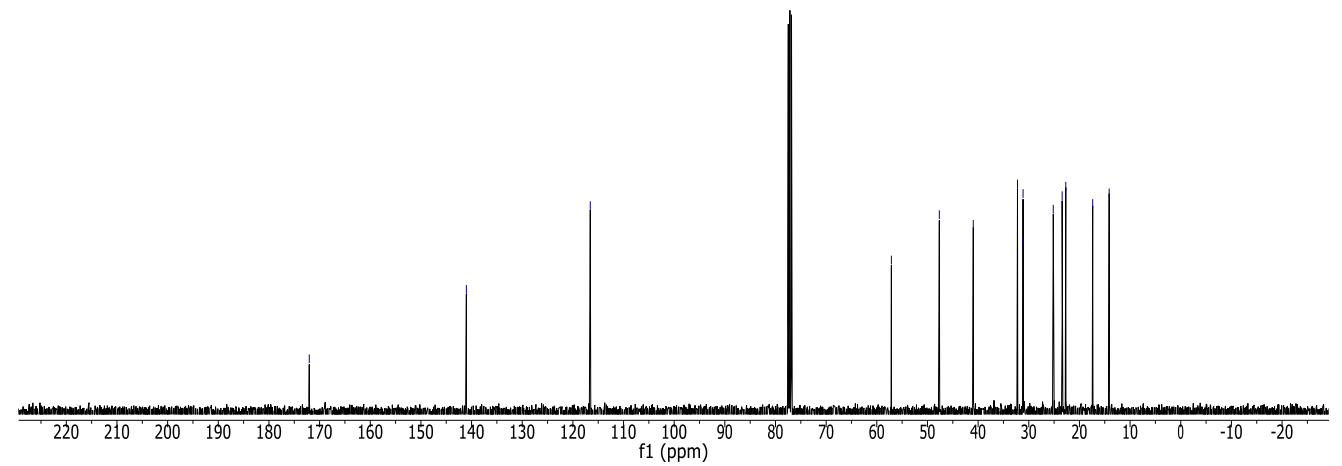




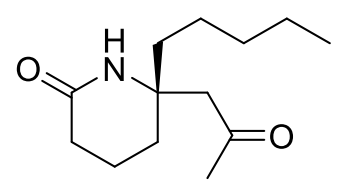

6
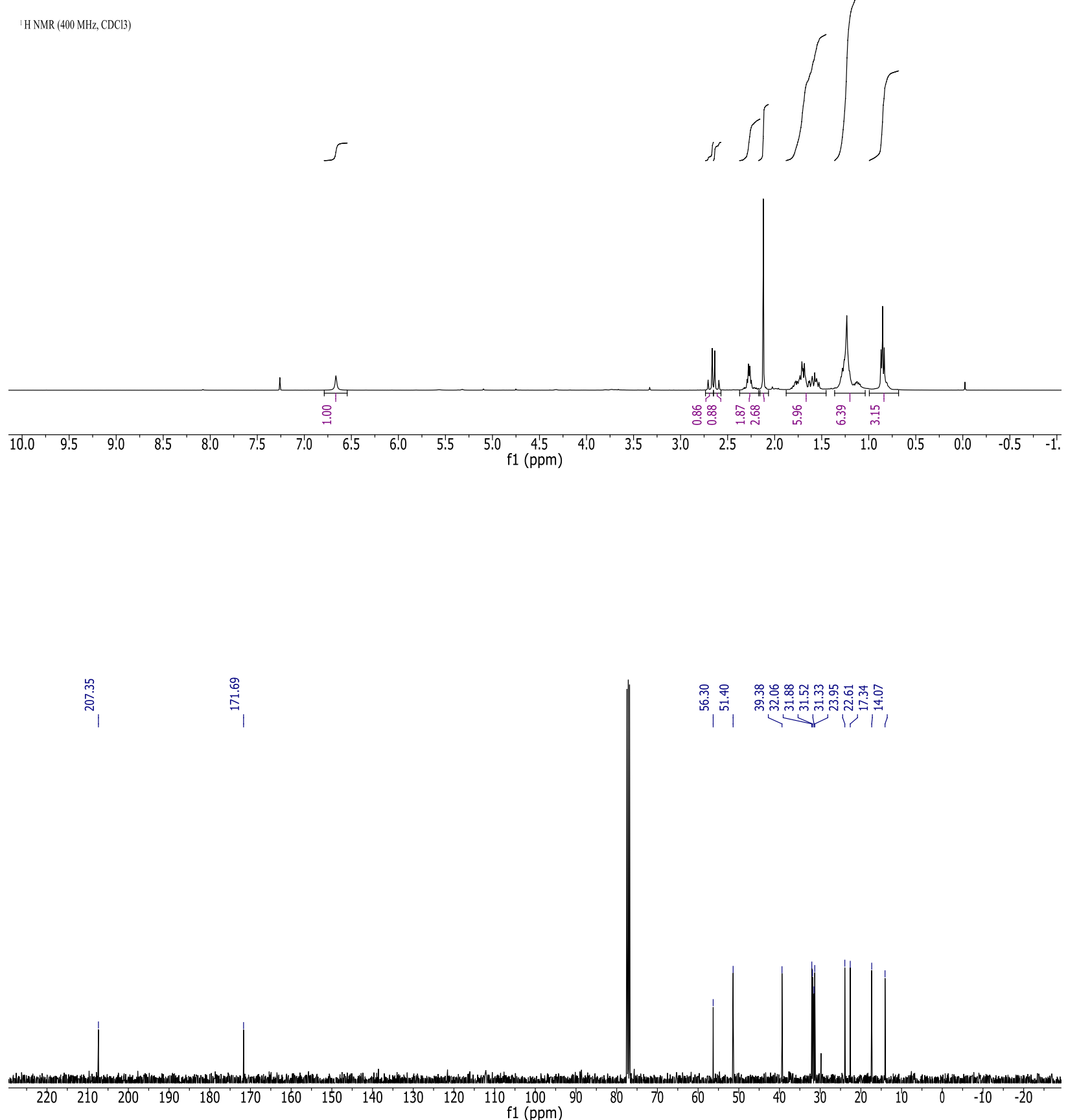